\title{
Versuche zum dynamischen Verhalten teilweise vorgespannter Leichtbeton- und Betonbalken
}

\section{Working Paper}

\section{Author(s):}

Gisin, Walter; Bachmann, Hugo

Publication date:

1985

Permanent link:

https://doi.org/10.3929/ethz-a-000362852

Rights / license:

In Copyright - Non-Commercial Use Permitted

Originally published in:

Bericht / Institut für Baustatik und Konstruktion ETH Zürich 7501(2) 
Versuche zum dynamischen Verhalten teilweise vorgespannter Leichtbetonund Betonbalken

Walter Gisin

Hugo Bachmann 


\section{CIP-Kurztitelaufnahme der Deutschen Bibliothek}

\section{Gisin, Walter:}

Versuche zum dynamischen Verhalten teilweise vorgespannter Leichtbeton- und Betonbalken / von Walter Gisin; H. Bachmann.- Basel; Boston; Stuttgart: Birkhäuser, 1985.

(Bericht/ Institut für Baustatik und Konstruktion ETH Zürich; Nr. 7501-2)

ISBN 3-7643-1758-2

NE: Bachmann, Hugo:; Institut für Baustatik und Konstruktion <Zürich > : Bericht

Nachdruck verboten.

Alle Rechte, insbesondere das der Übersetzungen in fremde Sprachen und der Reproduktion auf photostatischem Wege oder durch Mikrofilm, vorbehalten. 


\section{Versuche zum dynamischen Verhalten teilweise vorgespannter Leichtbeton- und Betonbalken}

von

Dipl. Ing. Walter Gisin

Prof. Dr. H. Bachmann

Institut für Baustatik und Konstruktion

Eidgenössische Technische Hochschule Zürich 
1. Einleitung 1

1.1 Allgemeines 1

1.2 Zielsetzung

1.3 Theoretische Grundlagen $\quad 2$

\begin{tabular}{ll}
1.4 Resultate der 1. Versuchsserie an schlaff bewehrten Balken & 2 \\
\hline
\end{tabular}

1.5 Entwickelte Dämpfungsmodelle für Stahlbetonbalken

2. Versuchsbalken $\quad 5$

2.1 Beschreibung der Versuchsbalken $\quad 5$

2.1.1 Abmessungen, statische Systeme

2.1.2 Bewehrung

2.1.3 Herstellung und Lagerung $\quad 6$

2.1 .4 Vorspannen und Injizieren $\quad 6$

2.1 .5 Zusatzmassen

2.2 .1 Leichtbeton und Beton 7

2.2.2 Injektionsgut 8

2.2.3 Schlaffer Bewehrungsstahl

$\begin{array}{ll}2.2 .4 \text { Spannstahl } & 8\end{array}$

$\begin{array}{ll}2.3 \text { Rechnerische Werte für die Versuchssteuerung und -auswertung } & 8\end{array}$

2.3.1 Materialkennwerte $\quad 8$

2.3.2 Querschnittswerte, Biegesteifigkeiten

$\begin{array}{lr}\text { 2.3.3 Biegemomente } & 9 \\ \text { 2.3.4 Spannungen } & 11\end{array}$

2.3.5 Vorspanngrade

3. Versuchsdurchführung 12

3.1 Versuchsanlage $\quad 12$

3.1.1 Dynamische Belastungseinrichtung 12

3.1.2 Statische Belastungseinrichtung

$\begin{array}{lc}\text { 3.1.3 Messeinrichtung } & 12 \\ \text { 3.1.4 Lager } & 13\end{array}$

3.2 Versuchsablauf 14

$\begin{array}{lr}3.3 \text { Versuchsauswertung } & 15\end{array}$

3.3.1 x-t-Thermo-Schreiber $\quad 15$

$\begin{array}{lr}\text { 3.3.2 Magnetband } & 15 \\ \text { 3.3.3 Datenerfassungsanlage } & 16\end{array}$

4. Versuchsresultate 17

$\begin{array}{ll}4.1 \text { Biegemomente, Spannungen } & 17\end{array}$

$\begin{array}{lr}4.2 \text { Biegesteifigkeiten } & 18\end{array}$

4.2.1 Statische Biegesteifigkeiten $\quad 18$

4.2.2 Dynamische Biegesteifigkeiten $\quad 20$

$\begin{array}{ll}4.3 \text { Eigenfrequenzen } & 22\end{array}$

$\begin{array}{ll}4.4 \text { Beschleunigungen } & 25\end{array}$

4.5 Dämpfung $\quad 26$

$\begin{array}{lr}\text { ZUSAMMENFASSUNG } & 29\end{array}$

RESUME

\begin{tabular}{lr} 
SUMMARY & 35 \\
\hline
\end{tabular}

$\begin{array}{lr}\text { VERDANKUNGEN } & 38\end{array}$

$\begin{array}{lr}\text { LITERATURVERZEICHNIS } & 39\end{array}$

$\begin{array}{lr}\text { BEZEICHNUNGEN } & 40\end{array}$

\begin{tabular}{lr} 
BILDER & 43 \\
\hline TABELLEN & 86
\end{tabular}

$\begin{array}{lr}\text { TABELLEN } & 86\end{array}$ 


\section{Einleitung}

\subsection{Allgemeines}

Neue und besser ausnützbare Materialien und neue Technologien ermöglichen den Bau immer schlankerer Tragwerke, die vor allem durch periodisch wirkende Kräfte leicht in Schwingungen mit grösseren Amplituden versetzt werden können. Insbesondere bei Verwendung von Leichtbeton lassen sich in Kombination mit der Vorspannung ausgesprochen schlanke Konstruktionen erstellen, wobei heute nicht mehr die volle, sondern die teilweise Vorspannung mit Rissbildung im Gebrauchszustand [5], [6] im Vordergrund steht.

Für die dynamische Analyse von Tragwerken stehen zahl reiche hochentwickel te Berechnungsverfahren und Computerprograme zur Verfügung. Wirklichkeitsnahe Rechenergebnisse können jedoch nur bei Kenntnis der dynamischen Materialeigenschaften wie z.B. dynamische Biegesteifigkeiten, Dämpfungswerte, usw. erzielt werden. Entsprechende Grundlagen auf dem Gebiet des teilweise vorgespannten Leichtbetons wie auch des teilweise vorgespannten Betons fehlen jedoch praktisch vollständig.

Zur Klärung des Einflusses einer teilweisen Vorspannung auf das dynamische Verhalten von Leichtbeton- und Betonkonstruktionen sind am Insti tut für Baustatik und Konstruktion (IBK) der Eidgenössischen Technischen Hochschule Zürich (ETH) im Rahmen des Forschungsprojektes 'Dynamisches Verhalten von Leichtbeton- und Betonkonstruktionen' Versuche an Leichtbeton- und Betonbalken durchgeführt worden. Beim vorliegenden Versuchsbericht handelt es sich um eine Fortsetzung der 1979 veröffentlichten 1. Versuchsserie an schlaff bewehrten Leichtbeton- und Betonbalken [1]. Mit der 1. Versuchsserie konnten grundlegende Erkenntnisse ubber den Einfluss der Rissbildung, der Beanspruchungshöhe, des Bewehrungsgehal tes sowie der Betonart (Leichtbeton, Normalbeton) auf Bauwerkseigenschaften wie Biegesteifigkeit, Eigenfrequenz und Dämpfung im elastischen Beanspruchungsbereich gewonnen werden. Entsprechende Berechnungsmodelle sind 1981 in einer theoretischen Arbeit [2] veröffentlicht worden.

Um direkte Vergleiche zwischen der 1. Versuchsreihe an schl aff bewehrten Balken und der hier beschriebenen 2. Versuchsreihe an teilweise vorgespannten Balken zu ermöglichen, wurden die Versuchsanlage und die Versuchsbalken in bezug auf Abmessungen, Spannweiten und Betonqualităt weitgehend analog konzipiert (Bilder 1 und 2).

\section{$1.2 \quad$ Ziel setzung}

Die vorliegende experimentelle Untersuchung dient zur Erforschung des Einflusses

- des Vorspanngrades

- der Rissbildung

- der Beanspruchungshöhe (Momente und Normalkraft bzw. Durchbiegung, Stahlbeanspruchung)

auf die dynamischen Eigenschaften von voll und teiTweise vorgespannten Leichtbeton- und Betonbalken unter Biegebeanspruchung. Dabei interessieren insbesondere

- die Biegesteifigkeit

- die Eigenfrequenz

- das Dämpfungsverhalten

- der Vergleich mit analogen Leichtbeton- und Betonbalken

im quasi-elastischen Beanspruchungsbereich. Ferner sollen anhand der Versuchsresultate die für die schlaff bewehrten Balken erarbeiteten theoretischen Modelle [2] überprüt werden. Die Versuchsresultate sind Grundlage für eine Erweiterung dieser Modelle auf teilweise vorgespannte Leichtbeton- und Betonbalken. 
Eine umfassende Zusammenstellung der theoretischen Grundlagen ist in [1] vorhanden.

\subsection{Resul tate der 1 . Versuchsserie an schlaff bewehrten Balken}

Im folgenden werden die wichtigsten Resultate der dynamischen Versuche an schlaff bewehrten Balken kurz zusammengestellt. Für ein eingehendes Studium sei auf [1] verwiesen.

\section{Biegemomente und Querkräfte}

Die Biegemomente und Querkräfte infolge dynamischer Relativbeanspruchung waren bei den entsprechenden Leichtbeton- und Betonbalken bei gleicher Relativdurchbiegung stets annähernd gleich gross, da sich die Ełnflüsse der höheren Beschleunigungen und der geringeren Masse der Leichtbetonbalken etwa kompensierten.

\section{Biegesteifigkeiten}

Die durch die Rissbildung bewirkte Abminderung der statischen und der dynamischen Biegesteifigkeit betrug gegenüber dem ungerissenen Zustand bei den Leichtbetonbalken $57 \%$ bis $71 \%$ und bei den Betonbalken $68 \%$ bis 84\%. Die Abminderung war bei schwach bewehrten Balken erwartungsgemäss grösser als bei stärker bewehrten Bal ken.

\section{Eigenfrequenzen}

Die Eigenfrequenzen der Versuchsbalken reduzierten sich mit zunehmender Rissbildung. Die Abnahme gegenüber dem gerissenen Zustand betrug bei den Leichtbetonbalken $33 \%$ bis $44 \%$ und bei den Betonbalken $47 \%$ bis $54 \%$. Die im ungerissenen Zustand kleineren Eigenfrequenzen der Leichtbetonbalken nahmen mit zunehmender Beanspruchung und Rissbildung weniger stark ab als die Eigenfrequenzen der analogen Betonbalken, dies trotz geringerer Masse infolge des wesentlich geringeren E-Moduls. Schliesslich wiesen sämtliche Leichtbetonbalken höhere Eigenfrequenzen auf als die analogen Betonbalken.

\section{Dämpfung}

Es wurde eine ausserordentlich starke Abhängigkeit der Dämpfung von der Beanspruchungshöhe (Grösse der Relativbeanspruchung) festgestellt. Im Bild 3 sind die aus den ersten zehn Zyklen eines Ausschwingversuchs ermittel ten Dämpfungswerte (logarithmisches Dekrement $\vartheta$ bzw. Dämpfungsmass $\xi$ ) in Funktion der Relativdurchbiegung $w_{\text {rel }}$ dargestel $1 \mathrm{t}$.

Bef einer erstmaligen Steigerung der Relativbeanspruchung (1. Prüfphase) stieg bei allen Versuchsbalken während der Rissbildung die Dämpfung vorerst an. Nachdem annähernd alle Biegerisse ausgebil det waren, nahm die Dämpfung mit weiter steigender Relativbeanspruchung wieder ab. Bei hoher Relativbeanspruchung, d.h. bei Stahlspannungen bis nahe bei der Proportionalitătsgrenze, wurden für das Dämpfungsmass sehr niedrige Werte von teilweise unter $1 \%$ ermittelt. Bei Wiederholung der Ausschwingversuche (2. Prüfphase) an den nun bereits gerissenen Balken wurden bei kleinen Relativbeanspruchungen für das Dämpfungsmass sehr hohe Werte von bis zu $4 \%$ und mehr festgestellt. Bei hohen Relativbeanspruchungen wurden dann aber bei fast allen Balken wiederum Werte von weniger als $1 \%$ gemessen. Die Dämpfung der Leichtbetonbalken war stets geringer als diejenige der analogen Betonbalken. 


\subsection{Entwickelte Dämpfungsmodelle für Stahlbetonbalken}

Die im Abschnitt 1.4 beschriebenen Versuchsresultate zeigten, dass mit dem Modell der viskosen Dämpfung, mit dem üblicherweise die Dämpfung von Stahlbetonbalken zu erfassen versucht wird, das tatsăchliche Dämpfungsverhalten nicht zutreffend beschrieben werden kann. Im folgenden werden die in der Dissertation [2] entwickel ten wirklichkeitsnaheren Modelle kurz vorgestellt.

Zur Beschreibung der Dämpfungsphänomene in einem Balken ist grundsätzlich zwischen ungerissenen und gerissenen Biegeelementen zu unterscheiden (Bild 4 ).

Bei einem ungerissenen Biegeelement erfolgt die Energiedissipation sowohl in der Biegedruck- als auch in der Biegezugzone ausschl iesslich durch viskose Dămpfung.

Bei einem gerissenen Biegelement wird die Energie durch viskose Dämpfung in der Biegedruckzone und durch Reibungsdämpfung in der Biegezugzone dissipiert. Die Reibungsdämpfung entsteht 1 ängs der oberfiäche des Bewehrungsstahls. Längs dieser Oberfläche bauen sich infolge Relativverschiebungen zwischen dem Beton und dem Stahl die im Bild 5 dargestellten Verbundkräfte auf. Die Energiedissipation erfolgt durch Gleitbewegungen der gegen den Bewehrungsstab gepressten Betonzăhne zwischen den Sekundärrissen.

Durch Aneinanderreihen ungerissener und gerissener Biegeel emente und durch Superposition der Dämpfungsanteile der einzelnen Biegeel emente lässt sich das Dämpfungsmass des ganzen Stahlbetonbalkens ermitteln. Ein Vergleich des auf diese Art für den ganzen Balken numerisch ermittel ten Dämpfungsmasses mit der pauschalen, an Versuchsträgern gemessenen äquivalenten viskosen Dămpfung lässt auf den Anteil der viskosen Dämpfung sowie auf den Anteil der Reibungsdämpfung schliessen. Damit können entsprechende Modell parameter festgelegt werden [2].

\section{$1.6 \quad$ Versuchsprogram}

Die Versuchsanlage ist im Bild 1 dargestellt. Es wurden ein vorgespannter Leichtbeton- und ein vorgespannter Betonbalken gleicher Ausführung geprüft. Die Abmessungen und Bewehrungen sind aus Bild 2 ersichtlich.

Die dynamischen Eigenschaften der Balken (Biegesteifigkeit, Eigenfrequenz, Dämpfung) wurden mit Ausschwingversuchen ermittelt. Die Anregung erfolgte mit Hil fe eines servohydraul ischen Schwingers in Balkenmitte (Bild 15), der plötzlich abgestellt wurde. Mit einer statischen Belastungseinrichtung (Bilder 12, 13, 14) konnte zudem vor und nach den Ausschwingversuchen die statische Biegesteifigkeit in dem jeweils interessierenden Durchbiegungsbereich gemessen werden.

Da die Versuche zerstörungsfrei durchgeführt werden konnten, war es möglich, mehrere Einflussparameter am gleichen Versuchsbalken zu untersuchen. Damit konnte bei versuchsmăssig kleinem Aufwand ein umfassender Eindruck betreffend der Vorspanneinflüsse gewonnen werden. Das am Leichtbeton- und am Betonbalken in analoger Wei se durchgeführte Versuchsprogramm umfasste in wesentlichen die folgenden vier Phasen:

- Phase 1:

Ausschwingversuche am vol1 vorgespannten ungerissenen Balken mit reduzierter Spannweite und zentrischer Vorspannkraft. Variation der Vorspannkraft. Spannstäbe ohne Verbund.

- Phase 2:

Ausschwingversuche am voll vorgespannten ungerissenen Balken mit grosser Spannweitè und exzentrischer Vorspannkraft. Volle Rissbildung durch kurzfristige Wegnahme der Vorspannkraft. Ausschwingversuche am erneut voll vorgespannten Balken mit grosser Spannweite (Risse überdrückt). Spannstäbe ohne Verbund.

- Phase 3:

Ausschwingversuche an teilweise vorgespannten Balken mit grosser Spannweite und exzentrischer Vorspannkraft. Variation des Vorspanngrades durch Aufbringen von Zusatzmassen. Gekrümmtes Spannglied ohne Ver- 
bund.

- Phase 4:

Gleich wie Phase 3 , jedoch mit gekrümmtem Spannglied mit Verbund.

Die Einzelheiten der einzelnen Versuchsphasen sind in den Tabellen la bis 1d sowie in den Bildern 6a bis $6 c$ dargestellt. Die Begriffe 'volle Vorspannung', 'teilweise Vorspannung' und 'Vorspanngrad' werden im Abschnitt 2.3.5 erk1ärt. 
2. Versuchsbalken

2.1 Beschreibung der Versuchsbalken

\subsubsection{Abmessungen, statische Sys teme}

Die Abmessungen und die statischen Systeme gehen aus den Bildern 2 und 6 hervor. Die Versuchsbalken mit einer Gesamtlänge von $1=8.40 \mathrm{~m}$, einer Brefte $b=0.40 \mathrm{~m}$ und einer Höhe $\mathrm{h}=0.24 \mathrm{~m}$ entsprachen in thren Abmessungen den schlaff bewehrten Balken der 1. Versuchsserie dieses Forschungsprojektes [1].

In der Phase 1 konnte mit einer reduzierten Spannweite $1=5.04 \mathrm{~m}$ in den als einfache Balken mit auskragenden Enden gelagerten Versuchsbalken die Biegebeanspruchung in Balkenruhelage gering gehalten werden. Damit genügte bereits eine kleine zentrische Vorspannkraft, um die Rissbildung sowohl in Balkenruhelage als auch bei begrenzten dynamischen Relativbeanspruchungen zu vermeiden. Dank der reduzierten Spannweite in der Phase 1 konnten im Vergleich zur Phase 2 etwa die doppelten Eigenfrequenzen bis $\mathrm{zu} 12 \mathrm{~Hz}$ erreicht werden, so dass sich für die voll vorgespannten Balken insgesamt ein breiter Frequenzbereich untersuchen liess.

Mit der für die Phasen 2, 3 und 4 gewählten Spannweite $1=7.92 \mathrm{~m}$ lagen die Eigenfrequenzen (abhängig von der Spannweite, der Masse und der Biegesteifigkeit bzw. dem Vorspanngrad) im Bereich von 2 bis $6 \mathrm{~Hz}$. Dies entspricht im Brücken- und im Hochbau oft vorkommenden Grundfrequenzen und ermöglicht praxisnahe Folgerungen.

\subsubsection{Bewehrung}

Die Anordnung der schlaffen Bewehrung und der Spannbewehrung geht aus Bild 2 hervor. Die Leichtbetonbalken und die Betonbalken waren identisch bewehrt:

- schlaffe Längsbewehrung: $2 \times 4$ Durchmesser $12 \mathrm{~mm}$

- Bügelbewehrung: Durchmesser $8 \mathrm{~mm}, t=150 \mathrm{~mm}$

- gerade, nicht injizierbare Spannstäbe in den Phasen 1 und 2: 6 Durchmesser $12 \mathrm{~mm}$

- parabelförmig gekrümmtes, injizierbares Spannglied in den Phasen 3 und 4: 7 Durchmesser $7 \mathrm{~mm}$

\section{Schl affe Bewehrung}

Mit je 4 Durchmesser $12 \mathrm{~mm}$ auf der Balkenoberseite und auf der Balkenunterseite $\left(\rho=\rho^{\prime}=0.57 \%\right.$ ) entsprach die schlaffe Längsbewehrung der Bewehrung der Balken mit kleinstem Bewehrungsgehalt LB 1 bzw. B 1 der 1. Versuchsreihe [1]. Damit war einerseits in der nicht vorgespannten Teilphase 2.2 ein ausreichender Biegewiderstand vorhanden, und andererseits wurde ein direkter Vergleich mit der 1. Versuchsreihe möglich. Ferner entsprach der gewähl te schlaffe Bewehrungsgehalt einem für teilweise vorgespannte Balken praxisnahen Wert.

Die Bügelbewehrung war so stark, dass im vornherein ein Schubbruch ausgeschlossen werden konnte. 
Spannstäbe

Für die Phasen 1 und 2 (volle Vorspannung) wurde ein Vorspannsystem gewählt, das eine Veränderung der Vorspannkraft zwischen den einzelnen Teilphasen erlaubt und selbst möglichst eine geringe Dämpfung verursacht. Dies konnte durch die Wahi gerader, eingefetteter, nicht injizierbarer Spannstäbe mit Durchmesser $12 \mathrm{~mm}$ weitgehend erreicht werden.

Die sechs Spannstäbe erlaubten zentrische Betonspannungen bis zu $8.3 \mathrm{~N} / \mathrm{mm} 2$ (vgl. Tabelle 2). Mit vier Spannstäben (2 zentrisch, 2 unten) war bei grosser Spannweite in der Phase 2 in Balkenruhelage ein Vorspanngrad $\kappa_{0}=1.93$ (Bal kenmitte) für den Leichtbetonbalken und $\kappa_{0}=1.55$ für den Betonbalken ( $\kappa_{0} v g l$. Tabelle 3 und Abschnitt 2.3.5) möglich, so dass für dynamische Relativbeanspruchung im Bereich der vollen Vorspannung genügend Reserve blieb. Die Verankerung der Spannstäbe erfolgte über eine Gewindemutter auf die Ankerplatte.

\section{Spannglied}

In den Phasen 3 und 4 (teilweise Vorspannung) sollte ein Balken mit einem in der Praxis üblichen Spanngliedverlauf untersucht werden. Das gewähl te parabelförmig eingelegte Spannglied (Typ BBRV 360 mit 7 Durchmesser $7 \mathrm{~mm}$ Paral. leldrahtbünde1) ermöglichte zentrische Betonspannungen bis $2.5 \mathrm{~N} / \mathrm{mm} 2$ (vgl. Tabelle 2). Der Vorspanngrad in Balkenruhelage betrug ohne Zusatzmassen $\kappa_{0}=1.45$ (Balkenmitte) für den Leichtbetonbalken und $\kappa_{0}=1.17$ für den Betonbalken ( $\kappa_{0}$ vgl. Tabelle 3 und Abschnitt 2.3.5). In Kombination mit den Spannstäben konnte der Vorspanngrad erhöht werden (Teilphase 2.4), in Kombination mit Zusatzmassen konnte er reduziert werden (Teiliphasen 3.2 bis 3.5 und Teilphasen 4.2 bis 4.5 ).

\subsubsection{Herstellung und Lagerung}

Die Versuchsbalken wurden in einer konventionell en Hol zschalung hergestellt. Durch Vornässen der Leichtzuschl agstoffe konnte deren Wasseraufnahme mit entsprechender Versteifung des Leichtbetons nach dem Mischen reduziert werden. Die Verdichtung erfolgte mit Hilfe eines Nadelvibrators. Nach vier Tagen wurden die Balken ausgeschalt und zwecks Verhinderung früher Schwindrisse zentrisch auf $2.2 \mathrm{~N} / \mathrm{mm} 2$ vorgespannt. Die Lagerung erfolgte bei einer Raumtemperatur von $20 \mathrm{Grad} C$ anfänglich im Betonierraum, später in der Forschungshal le der ETH-Hönggerberg, wo auch die weiteren Vorbereitungsarbeiten wie Aufkleben der Messbolzen, Dehnmessrahmen, usw. durchgeführt wurden.

Um den Einfluss des Alters auf die Dämpfung von Leichtbeton und Beton gering hal ten zu können, begannen die Prüfungen erst nach einer Lagerung der Balken von 40 Tagen. Versuche an Mörtel- und Betonproben [3] haben gezeigt, dass nach dieser Zeit nur noch mit einer unwesentlichen al tersbedingten Veränderung der Dămpfung zu rechnen ist.

\subsubsection{Vorspannen und Injizieren}

Eine Zusammenstellung der Vorspannkräfte ist in der Tabelle 2 gegeben. Beim Einbauen des Balkens in die Versuchsanlage mit reduzierter Spannweite (Phase 1) waren zwecks Verhinderung früher Rissbildung alle sechs Spannstäbe auf ca. $0.2 \mathrm{f}_{\mathrm{pt}}$ vorgespannt. Das Paralleldrahtbündel mit 7 Durchmesser $7 \mathrm{~mm}$ war zu diesem Zeitpunkt noch nicht in das Hüllrohr eingeführt. Die Vorspannkraft in den Spannstăben wurde ausschliesslich mit Hilfe einer Zentrumslochpresse einzeln auf jeweils $0.4 f_{p t}$ (Teil phase 1.1 ), $0.6 f_{p t}$ (Teilphase 1.2) und $0.75 \mathrm{f}_{\mathrm{pt}}$ (Teil phase 1.3) erhöht. Beim Umrüsten auf die Versuchsanlage mit grosser Spannweite waren, um Rissbildung zu vermeiden, alle sechś Spannstäbe auf $0.75 \mathrm{f}_{\text {pt }}$ vorgespannt.

Das Paralleldrahtbündel wurde nach der Teilphase 3.2 durch das parabelförmig eingelegte Hüllrohr gestossen. Nach dem nachtrăglichen Aufstauchen der Ankerköpfchen auf der einen Seite erfolgte die Vorspannung

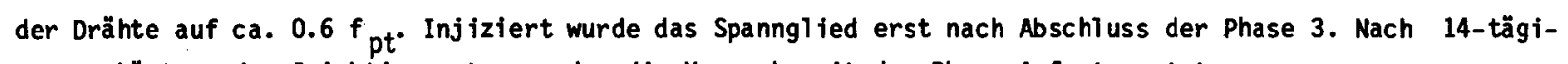
ger Aushärtung des Injektionsgutes wurden die Versuche mit der Phase 4 fortgesetzt. 
Die Kraftmessdosen erlaubten eine ständige Kontrolle der Vorspannkraft in den Spannstäben sowie eine anfängliche Kontrolle der Vorspannkraft im Paralleldrahtbündel (Bild 16). Die Obertragung der Vorspannkraft auf den Beton erfolgte über eine speziell gebohrte Ankerplatte.

\subsubsection{Zusatzmassen}

Die Anordnung der Zusatzmassen ist im Bild 7 dargestellt. Die Gewichte der in den einzelnen Teilphasen aufgebrachten Zusatzmassen können der Tabelle 9 entnommen werden.

Mit Hilfe der Zusatzmassen konnte die Gesamtmasse auf mehr als den doppelten Wert der Eigenmasse des Balkens erhöht werden. Dies bewirkte eine Reduktion des Vorspanngrades in Balkenruhelage auf $k_{0}=0.67$ (Teilphase 3.5, Teil phase 4.5 Balkenmitte) beim Leichtbetonbalken und auf $\kappa_{0}=0.61$ beim Betonbalken ( $\kappa_{0} \mathrm{vgl}$. Tabelle 3 und Abschnitt 2.3.5).

Im Leichtbetonbalken konnten durch entsprechende Zusatzmassen in den Teilphasen 3.2 und 4.2 bzw. 3.4 und 4.4 dieselben Vorspanngrade $k_{0}$ erreicht werden wie im Betonbalken in den Teilphasen 3.1 und 4.1 bzw. 3.3 und 4.3. Damit war ein direkter Vergleich möglich.

Die Zusatzmassen bestanden aus Blejplatten mit den Abmessungen $40 \times 220 \times 220 \mathrm{~mm} \mathrm{zu}$ je $20 \mathrm{~kg}$ Masse. Diese wurden mit $\mathrm{Hil}$ fe einbetonierter Verankerungsdübel und darin eingeschraubter Gewindestangen in Paketen zu maximal $407 \mathrm{~kg}(2 \times 10 \mathrm{Bleiplatten}$ plus Stahlunterlagen und Gewindestangen) auf dem Versuchsbalken zugfest befestigt. Eine Erhöhung der Biegesteifigkeit konnte durch diese Befestigungsart ausgeschiossen werden.

\subsection{Baustoffe}

\subsubsection{Leichtbeton und Beton}

Die Zusanmensetzung des Leichtbetons und des Betons wurde gleich gewăhlt wie in der 1. Versuchsreihe dieses Forschungsprojektes und kann der Tabelle 4 entnommen werden.

Das Mischrezept für den Leichtbeton stanmte von der Firma Hunziker \& Cie AG, Zürich. Als Leichtzuschlagstoff wurde Blähton Leca-hade, getrennt nach den Korngrössen $3 \mathrm{~mm}$ bis $8 \mathrm{~mm}$ und $8 \mathrm{~mm}$ bis $15 \mathrm{~mm}$, zugegeben. Als Bindemittel diente normaler Portl andzement. Zur Verbesserung der Verarbeitbarkeit des Leichtbetons wurde hydraul ischer Kalk beigemengt.

Die Zuschlagstoffe des Betons mit maximalem Korndurchmesser von $32 \mathrm{~mm}$ bestanden aus rolligem gewaschenem Material. Die Siebkurve entsprach ungefähr der EMPA-Kurve. Als Bindemittel diente normaler Portl andzement.

Zur Ermittlung der Betonkennwerte wurden mit jedem Balken vier Würfel mit $150 \mathrm{~mm}$ Seitenlänge sowie zwei Zylinder $150 \times 300 \mathrm{~mm}$ hergestellt. Bei einem Probealter von 34 Tagen (Leichtbeton) bzw. 29 Tagen (Beton) wurden an den Würfeln die Druckfestigkeit und das Raumgewicht und an den Zylindern der Elastizi tätsmodul und die Prismendruckfestigkeit bestimmt. Die Resultate sind in der Tabelle 5 zusammengestellt.

Die Würfeldruckfestigkeit wurde mit einer Dehngeschwindigkeit $\dot{\varepsilon}=0.0022 /$ min ermittelt. Der Elastizi tätsmodul murde an den Zylindern als Sekantenmodul im Spannungsintervall von $5 \mathrm{~N} / \mathrm{mm} 2$ bis $10 \mathrm{~N} / \mathrm{mm} 2\left(E_{5-10}\right)$ und von $5 \mathrm{~N} / \mathrm{mm} 2$ bis $20 \mathrm{~N} / \mathrm{mm} 2\left(E_{5-20}\right)$ bestimmt. Nach jeweils fünfmaliger Vorbelastung von 0 bis $10 \mathrm{~N} / \mathrm{mm} 2 \mathrm{bzw}$. 0 bis $20 \mathrm{~N} / \mathrm{mm} 2$ wurde mit einer Dehngeschwindigkeit von $\dot{\varepsilon}=0.0001 /$ min die Belastung kraftgesteuert von 5 auf $10 \mathrm{~N} / \mathrm{mm} 2 \mathrm{bzw}$. von 5 auf $20 \mathrm{~N} / \mathrm{mm} 2$ gesteigert. Die Dehnungen wurden auf einer Messiănge von $100 \mathrm{~mm}$ ermittel t. Anschliessend murden die Zylinder verformungsgesteuert bis zum Bruch belastet. Die erhaltenen Spannungs-Dehnungs-Diagramme sind im Bild 8 dargestel1t. 


\subsubsection{Injektionsgut}

Die Zusammensetzung des vom Spanngliedlieferanten hergestellten Injektionsgutes ist aus der Tabelle 4 ersichtlich. Als Quellmittel murde Intracrete beigemischt. Die Festigkeit des Injektionsgutes wurde an $Z y-$ lindern vom Durchmesser $100 \mathrm{~mm}$ ermittelt. Die erreichten Werte können der Tabelle 5 entnommen werden.

\subsubsection{Schl affer Bewehrungsstahl}

Die schlaffe Längs- und Bügelbewehrung bestand in Analogie zur 1. Versuchsreine dieses Forschungsprojektes aus kal tverformtem hochwertigem Bewehrungsstahl (TORIP).

Da die Balken nicht bis zum Bruch beansprucht wurden, konnte auf eine spezielle Prüfung des Stahls verzichtet werden. Die garantierten Festigkeitswerte des Stahllieferanten können der Tabelle 6 entnommen werden.

Da in der 1. Versuchsserie an nur schlaff bewehrten Balken bei wesentlich mehr Lastwechselzahlen im Bereich grosser Spannungsamplituden (keine Vorspannung) keine Ermüdungsbrüche eintraten, durften solche auch im vornherein für die vorliegenden Versuche ausgeschlossen werden.

\subsubsection{Spannstah1}

Es wurden sowohl Spannstäbe als auch Paralleldrahtbündel und Verankerungen vom Typ BBRV verwendet. Die Spannstäbe mit Durchmesser $12 \mathrm{~mm}$ und die Drähte mit Durchmesser $7 \mathrm{~mm}$ wiesen eine glatte Oberfläche auf. Da die Balken nicht bis zum Bruch beansprucht wurden, genügten auch beim Spannstahl die garantierten Festigkeitsangaben des Spannstahllieferanten, die der Tabelle 6 zu entnehmen sind.

Ermüdungsbrüche durften auch für den Spannstahl aufgrund der in [4] wiedergegebenen Versuchsresul tate ausgeschlossen werden.

\subsection{Rechnerische Werte für die Versuchssteuerung und -auswertung}

Um in den Phasen 1 und 2 (exklusive Teilphase 2.2) die Bedingungen für die volle Vorspannung einzuhalten, und um zu verhindern, dass in den Phasen 3 und 4 sowie in der Teilphase 2.2 die Spannungen den linear elastischen Bereich überschritten, mussten vor Versuchsbeginn mit Hilfe rechnerischer Werte die Versuchssteuergrössen der einzelnen Teilphasen festgelegt werden. Als Versuchssteuergrösse diente die Relativdurchbiegung nach unten in Balkenmitte, $w_{\text {rel }}^{+}$. Die gewăhl ten Maximalwerte $w_{\text {rel, }}^{+}$max sind in der Tabelle 12 dargestellt.

\subsubsection{Material kennwerte}

Um die Versuchssteuergrössen rechtzeitig festlegen zu können, mussten die Materialkennwerte der Leichtbeton- und der Betonproben ca. zehn Tage vor Versuchsbeginn bestimmt werden. 
E-Moduli zur Berechnung der ideellen Querschnittswerte

\begin{tabular}{ll}
\hline Leichtbeton: & $E_{c}=18^{\prime} 500 \mathrm{~N} / \mathrm{mm} 2$ \\
Beton: & $E_{c}=30^{\prime} 000 \mathrm{~N} / \mathrm{mm} 2$ \\
Sch1 affer Stah1 : & $E_{s}=210^{\prime} 000 \mathrm{~N} / \mathrm{mm} 2$ \\
Spannstahl mit Verbund: & $E_{p}=210^{\prime} 000 \mathrm{~N} / \mathrm{mm} 2$ \\
Spannstahl ohne Verbund: & $E_{p}=126^{\prime} 000 \mathrm{~N} / \mathrm{mm} 2$
\end{tabular}

Da sich Zusatzdehnungen beim Spannstahı ohne Verbund auf die ganze Lănge der Stäbe verteilten, wurde der E-Modul für diesen Fal 1 mit dem Faktor 0.6 (Schätzung) reduziert.

\section{Biegezugfestigkeit zur Berechnung des Rissmomentes}

Aufgrund der Versuchsresul tate der 1. Versuchsreihe [1] konnten näherungsweise folgende rechnerische Biegezugfestigkeiten angenommen werden:

$\begin{array}{ll}\text { Leichtbeton: } & f_{c t}=3 \mathrm{~N} / \mathrm{mm} 2 \\ \text { Beton: } & f_{c t}=3 \mathrm{~N} / \mathrm{mm} 2\end{array}$

\section{Festigkeitswerte zur Berechnung des plastischen Momentes}

Diese Werte wurden den jeweiligen Mittelwerten entsprechend aus der Prüfung der Leichtbeton- und der Betonproben bzw. den garantierten Werten der Stahllieferanten entsprechend angenommen. Der Spannungszuwachs bis zum Bruch des Balkens wurde beim Spannstahl ohne Verbund mit $200 \mathrm{~N} / \mathrm{mm} 2$ geschătzt.

\subsubsection{Querschnittswerte, Biegesteifigkeiten}

Die rechnerischen Querschnittswerte sowie die Biegesteifigkeiten können den Tabellen 7 und 8 sowie Bild 10 entnormen werden. Alle Werte gelten für die Balkenmitte. Die sich von Teilphase zu Teilphase verändernden Querschnittstypen sind in Bild 9 dargestellt.

Bei der Berechnung der Querschnittswerte wurde angenomen, dass die mittlere über einem Biegerissel ement vorhandene Stahldehnung $\varepsilon_{\mathrm{sm}}$ und die maximale im Riss auftretende Stahldehnung $\varepsilon_{\mathrm{s} \text {, max }}$ gleich gross sind. Der rechnerische Verbundkoeffizient $\bar{k}$ betrug somit

$$
\bar{K}=\frac{\varepsilon_{\text {Sm }}}{\varepsilon_{S, \max }}=1.0
$$

Diese Annahme widerspricht zwar dem zu erwartenden Verbundverhal ten, doch lassen sich damit $k l$ are rechnerische Bezugswerte ermitteln, was die Interpretation der Versuchsresul tate erleichtert.

\subsubsection{Biegemomente}

Biegemomente in Balkenruhel age

Die rechnerischen Biegemomente in Balkenruhel age, Mop, ergaben sich aus dem gemessenen Raumgewicht der Materialproben, aus dem Gewicht des servohydraulischen Schwingers, der Ankerplatten und der Zusatzmassen (Tabelle 9) sowie aus den Wirkungen der gemessenen Vorspannkräfte (Tabelle 2).

Es gilt:

$$
M_{o p}=M_{0}+M_{p}
$$

mit:

$M_{0}=$ Biegemoment in Balkenruhelage infolge Balkeneigengewichtes, Zusatzmassen, Schwinger und Ankerplatten

$M_{p}=$ Biegemoment infolge Vorspannung 
Die rechnerischen Biegemomente $M_{0}, M_{p}$ und $M_{o p}$ können der Tabelle 10 entnommen werden. Der Verlauf von $M_{o p}$ längs der Bal kenachse ist in den Bildern $21 \mathrm{a}$ bis $21 \mathrm{f}$ aufgezeichnet.

\section{Biegemomente infolge von Relativdurchbiegungen}

Die Biegemomente Mrel infolge von Relativdurchbiegungen ergaben sich aus der Eigenfrequenz, die ihrerseits ein Mass der Biegesteifigkeit ist. Der Zusammenhang von Eigenfrequenz und Steifigkeit lässt sich beim Einmas senschwinger rechnerisch beschreiben mit:

$$
f=\frac{1}{2 \pi} \sqrt{\frac{k}{m}}
$$

Darin ist $k$ die Federsteifigkeit und $m$ ist die Masse des Einmassenschwingers.

Für den Versuchsbalken mit konstanter Massenverteilung und einer Einzelmasse in Balkenmitte (servohydraulischer Schwinger) ergibt dies unter der Annahme konstanter Biegesteifigkeit längs der Balkenachse $E I=B=$ konstant:

$$
f=\frac{1}{2 \pi} \sqrt{\frac{B 1}{m+2 M}}
$$

Darin ist $m$ die Masse pro $m^{\prime}$ Balkenlänge (inkl. Zusatzmassen). M ist die Masse des servohydraulischen Schwingers, und 1 ist die Spannweite.

Der rechnerische Zusammenhang zwischen Biegesteifigkeit, Biegemoment und Relativdurchbiegung lässt sich unter der Annahme konstanter Biegesteifigkeit längs der Balkenachse für den einfachen Balken mit Hilfe baustatischer Methoden leicht ermitteln. Zusammen mit der obigen Beziehung kann dieser rechnerische Zusammenhang auf die Eigenfrequenz erweitert werden. Die Tabelle 11 enthält die gerechneten Zusarmenhänge für die einzelnen Teilphasen. Anhand dieser Tabelle konnten mit Hilfe rechnerischer Eigenfrequenzen durch Eingrenzung der Biegemomente die zulässigen Relativdurchbiegungen als Versuchssteuergrössen festgelegt werden (Tabel le 12).

Auf die in den Bildern $21 \mathrm{a}$ bis $21 \mathrm{f}$ dargestel1ten Biegemomente $M_{\text {rel }}$, basierend auf gemessenen Eigenfrequenzen, wird im Abschnitt 4.1 näher eingegangen.

\section{Bezugsgrössen für die Biegebeanspruchung}

- Dekompressionsmoment $\mathrm{M}_{\mathrm{DeC}}$ :

Das Dekompressionsmoment $M_{\text {Dec }}$ ist dasjenige Biegemoment aus Eigengewicht und Relativbeanspruchung, welches an einem vorgespannten Querschnitt aufgebracht werden muss; damit am spanngliednahen Rand die Betonspannung aus Vorspannung gleich null wird. Die aufgrund der gemessenen Vorspannkräfte berechneten Dekompressionsmomente können dem Bild 11 entnommen werden.

- Rissöffnungsmoment $M_{\text {ro }}$ :

Zur Definition von $M_{\text {ro }}$ ist die Wirkung der Vorspannung aufzuspalten in einen Normalkraftanteil $N_{p}$ und einen Biegeanteil $M_{p}$. Das Rissöffnungsmoment $M_{\text {ro }}$ ist die Summe aus dem Dekompressionsmoment $M_{\text {Dec }} p l u s$ Biegeanteil der Vorspannung $M_{p}$. Es gibt in den Bildern 21a bis $21 f$ an, ab welchem Gesamtmoment (Sume der Momente aus Eigengewicht, Vorspannung und Relativbeanspruchung) in Balkenmitte unten bzw. oben Betonzugspannungen auftreten bzw. bestehende Risse sich öffnen. Die aufgrund der gemessenen Vorspannkräfte berechneten Rissöffnungsmomente können dem Bild 11 entnommen werden.

- Rissmoment $M_{r}$ :

Das Rissmoment $M_{r}$ ist dasjenige Biegemoment aus Eigengewicht und Relativbeanspruchung, welches an einem 
Querschnitt (vorgespannt oder nicht vorgespannt) aufgebracht werden kann, damit die Betonspannungen am Zugrand gerade die Biegezugfestigkeit des Betons (Abschnitt 2.3.1) erreichen (Bild 11).

- Plastisches Moment $\mathrm{M}_{\mathrm{pl}}$ :

Das $p l$ astische Moment $M_{p l}$ ist dasjenige Biegemoment aus Eigengewicht und Relativbeanspruchung, welches aufgebracht werden kann, damit einer der Festigkeitswerte gemäss Abschnitt 2.3.1 gerade erreicht wird (Fliessen des Stahls).

\subsubsection{Spannungen}

Die Bilder $21 \mathrm{a}$ bis $21 \mathrm{f}$ zeigen in der rechten Spalte die rechnerischen Spannungen in Balkenmitte (Linie $\mathrm{m}-\mathrm{m}$ für Balkenruhelage). Diese wurden ausgehend von den Biegemomenten in Balkenmitte (mittlere Spalte, vgl. Abschnitt 2.3.3) mit Hilfe der Querschnittswerte gemäss Abschnitt 2.3.2 berechnet. Der Verbundkoeffizient wurde mit $\bar{k}=1.0$ angenommen.

\subsubsection{Vorspanngrade}

Im vorliegenden Bericht gelten für den Vorspanngrad folgende Definitionen:

- Vorspanngrad in Bal kenruhelage:

$$
\kappa_{0}=\frac{M_{D e c}}{M_{0}}
$$

- Vorspanngrad bei maximaler Relativdurchbiegung nach unten:

$$
K_{\text {rel, } \max }^{+}=\frac{M_{\text {Dec }}}{M_{0}+M_{\text {rel }}}
$$

- Vorspanngrad bei maximaler Relativdurchbiegung nach oben:

$$
K_{\text {rel, max }}^{-}=\frac{M_{\text {Dec }}}{M_{0}-M_{\text {rel }}}
$$

Für $K \geq 1.0$ treten am unteren Balkenrand keine Zugspannungen auf. Der Balken ist voll vorgespannt. Für 1.0 $\geq K \geq 0.0$ ist der Balken teilweise vorgespannt.

Die aufgrund der gemessenen Vorspannkräfte berechneten Vorspanngrade sind in der Tabelle 3 aufgeführt. Alle Werte beziehen sich auf den unteren Rand in Balkenmitte. 


\section{Versuchsdurchfuhrung}

\subsection{Versuchsanl age}

Das Bild 1 zeigt die Versuchsanlage auf dem Aufspannboden der Forschungshalle der ETH-Hönggerberg. Sie wurde, mit Ausnahme der Ausschwingvorrichtung und der statischen Belastungsvorrichtung, weitgehend von der 1. Versuchsreihe dieses For schungsprojektes [1] übernommen.

\subsubsection{Dynamische Bel astungseinrichtung}

Der für die Erzeugung der erzwungenen Schwingungen benützte servohydraulische Schwinger ist im Bild 15 abgebildet. Eine genaue Beschreibung ist im Anhang von [1] enthal ten.

Mit Hil fe eines durch eine Servohydraul ik gesteuerten Zug- und Druckzylinders wird in einem Rahmen eine Schwingmasse hin und her bewegt. Ein elektrisch gesteuertes Servoventil erlaubt einen relativ zum Balken sinusförmigen Bewegungsverlauf der Schwingmasse. Dank stufenloser Frequenzregelung von 0 bis $25 \mathrm{~Hz}$ und stufenloser Amplitudenregelung der Schwingmasse von 0 bis $100 \mathrm{~mm}$ sowie der Möglichkeit, die Schwingmasse zwischen 10 und $200 \mathrm{~kg}$ zu variieren, kann innerhalb der Leistungsgrenzen der Servohydraulik jede beliebige Kraft-Frequenz-Kombination erzeugt werden.

Der servohydraulische Schwinger wurde in Balkenmitte fest montiert. Die auf den Balken ausgeübten Kräfte entsprachen den durch die Hin- und Herbewegung der Schwingmasse erzeugten. Trägheitskräften.

\subsubsection{Statische Bel astungseinrichtung}

Zur Ermittlung der statischen Biegesteifigkeit wurde eine statische Belastungseinrichtung verwendet. Sie ist im Bild 12 schematisch dargestellt und in den Bildern 13 und 14 abgebildet.

Der Versuchsbalken wurde in zwei Punkten von je $s=1.12 \mathrm{~m}$ von der Balkenmitte belastet. Die Angriffspunkte der beiden Einzelkräfte waren so gewählt worden, dass sich statisch und dynamisch etwa derselbe Verlauf der Relativdurchbiegung längs der Balkenachse ergab. Die Einzelkrăfte murden mit Hilfe zweier Hohlkolbenpressen (abgestützt durch je einen auf dem Aufspannboden befestigten Sockel unter dem Versuchsbalken) über Druckstangen (für Relativdurchbiegungen nach oben) und Zugstangen (für Relativdurchbiegungen nach unten) aufgebracht. Zwischen Kolben und Druck- bzw. Zugstangen eingebạte Kraftmessdosen ermögl ichten eine Kraftmessung. Die Kraftübertragung in den Versuchsbalken erfolgte über je einen relativ leichten, den Balken umfassenden Stahl rahmen, der während den Ausschwingversuchen weggenormen wurde.

\subsubsection{Messeinrichtung}

Das Bild 20 zeigt den Messstellenplan. Als Messgeber wurden verwendet:

- induktive Wegaufnehmer zur Messung der Relativdurchbiegungen (in Balkenmitte und in einem Abstand von $1.5 \mathrm{~m}$ von der Balkenmitte) während des Ausschwingversuchs sowie während des statischen Bel astungsversuchs;

- eine versetzbare induktive Messstange zur punktweisen Ermittlung der Relativdurchbiegungen längs der Bal kenachse in Abständen von $500 \mathrm{~mm}$ während des statischen Bel astungsversuchs;

- ein Beschl eunigungsaufnehmer in Bal kenmitte; 
- Kraftmessdosen zur 1 aufenden Vorspannkraftmessung der Spannstäbe und zur Vorspannkraftmessung des Paralleldrahtbündels sowie zur Messung der Kolbenkräfte während des statischen Belastungsversuchs;

- ein Dreigelenk-Dehnmessrahmen mit einer Messstrecke von $200 \mathrm{~mm}$ Länge zur Messung der rel ativen Stahlund Betondehnungen während des Ausschwingversuchs sowie während des statischen Bel astungsversuchs.

Die Registrierung der Messignale während des Ausschwingversuchs erfolgte mit Hilfe

- eines Kathodenstrahloszillographen mit Speicher zur Aufzeichnung der Relativdurchbiegung in Balkenmitte (diente der Versuchssteuerung);

- eines x-t-Thermo-Schreibers (2 Kanäle) zur Aufzeichnung der Relativdurchbiegung und der relativen Betondehnung (Phasen 1 und 2) bzw. Stahldehnung (Phasen 3 und 4) in Balkenmitte;

- eines Magnetbandes (4 Kanäle) zur Auf zeichnung der Messignale der induktiven Wegaufnehmer (2 Kanäle) sowie zur Auf zeichnung der PCM-Signale;

- einer PCM-Anlage (PCM = Puls Code Modulation, 8 Kanäle) zur Erfassung, Digitalisierung und Uebertragung der Messignale aller Dreigelenk-Dehnmessrahmen sowie der Signale des Beschleunigungsgebers auf das Bandgerät.

Die Registrierung der Messignale während des statischen Belastungsversuchs erfolgte mit Hilfe

- einer el ektronischen Datenerfassungsaniage:

Die Messung der festverdrahteten Instrumente (alle Kraftmessdosen sowie der induktive Wegaufnehmer in Balkenmitte) erfolgte als automatische Ablesung in einem Block, die Messungen mit der versetzbaren induktiven Messstange mussten einzeln ausgelöst werden.

- schriftlicher Protollierung der Signalgrössen der Dreigelenk-Dehnmessrahmen.

\subsubsection{Lager}

Im Bild 17 ist das feste Lager und im Bild 18 ist das bewegliche Lager abgebildet. Die Lager wurden aus der 1. Versuchsreihe dieses Forschungsprojektes übernommen. Sie waren

- druck- und zugfest, um auch negative Auflagerkräfte während der dynamischen Relativbeanspruchung aufnehmen zu können;

- reibungsarm, spiel- und zwängungsfrei, um die Energiedissipation innerhalb der Lager möglichst gering hal ten zu können (praktisch keine Systemdämpfung).

\section{Fes tes Lager}

Die Auflagerdrehungen wurden durch zwei Pendelkugellager mit einem Reibungskoeffizienten von unter $2 \% 0$ ermöglicht. Beidseits des Balkens war je eine vertikale versteifte Stahlplatte angeordnet. Die beiden Platten wurden durch vier hochwertige Gewindestangen gegeneinander gespannt. Auf die Platten wurde je eine kurze Welle, die den inneren Ring des Kugellagers trug, geschweisst. Die äusseren Ringe der beiden Kugellager sassen in zwei weiteren Stahlplatten, die die Auflagerkräfte über ein Auflager an den Aufspannboden abgaben. 


\section{Bewegl iches Lager}

Das bewegliche Lager murde aus Stabilitätsgründen als hăngendes Pendellager ausgebildet, um eine bessere Zugănglichkeit zum Versuchskörper zu ermöglichen. Die Drehung um die Auflagerachse wurde durch eine analoge Konstruktion wie beim festen Lager ermöglicht. Die Längsverschieblichkeit war durch ein aus steifen Stahlplatten hergestelltes Pendel gewährleistet. Am oberen Ende des Pendels wurde ein Bronzelager angeordnet, das sich um eine an einem steifen Rahmen befestigte Welle drehen konnte. Wegen den sehr geringen Drehungen in diesem Lager konnte dort eine etwas grössere Reibung in Kauf genormen werden.

\subsection{Versuchsablauf}

Die Leichtbeton- und die Betonbalken murden ausgehend von verschiedenen Beanspruchungszuständen in Balkenruhelage geprüft. Diese konnten in den Phasen 1 und 2 durch Variation der Vorspannung, in den Phasen 3 und 4 durch Variation der Zusatzmassen verändert werden. Jede Teilphase entsprach einem eigenen Beanspruchungszustand in Balkenruhelage. Der Verlauf der Biegemomente lăngs der Balkenachse sowie die Spannungen in Balkenmitte sind für den Beanspruchungszustand in Balkenruhelage $\left(M_{0}\right)$ und für den Beanspruchungszustand bei maximalen Relativdurchbiegungen $\left(M_{0}+M_{r e l}\right)$ in den Bildern 21a bis $21 f$ dargestellt. Eine Beschreibung dieser Teilphasen ist in den Tabellen la bis $1 d$ sowie in den Bildern $6 a$ bis $6 c$ vorhanden.

Pro Teil phase wurden folgende Versuche durchgeführt:

- ein statischer. Belastungsversuch (Bilder 23a bis 23d) mit den gleichen Relativdurchbiegungen wie beim Ausschwingversuch. Damit konnte die Biegesteifigkeit in Funktion der statischen Relativdurchbiegung (Bilder 24a bis 24c) bestimmt werden.

- drei Ausschwingversuche mit jeweils denselben maximal en dynamischen Relativdurchbiegungen (Tabelle 12).

- ein statischer Bel astungsversuch.

In der Phase 1 wurden keine statischen Bel astungsversuche durchgeführt. Die Ausschwingversuche begannen jeweils eine Stunde nach Veränderung der Vorspannkraft bzw. nach Veränderung der Zusatzmassen.

In der Teilphase 1.3 folgte zusätzlich zur 1 . Serie nach zwei, vier, acht, vierundzwanzig und achtundvierzig Stunden je eine weitere Serie mit drei Ausschwingversuchen, um den Einfluss des Versuchszeitpunktes nach Veränderung der Vorspannkraft auf Eigenfrequenz und Dämpfung des ungerissenen Balkens zu erfassen.

Vor Beginn der Ausschwingversuche musste jeweils die Resonanzkurve durch schrittweise Veränderung der Anregungsfrequenz des servohydraulischen Schwingers abgetastet werden, um die Eigenfrequenz des Balkens in der jeweiligen Teilphase zu ermitteln. Als Anregungsfrequenz wurde anschliessend die gefundene Eigenfrequenz (Resonanzspitze) eingestellt. Eine stufenweise Erhöhung der Schwingeramplitude führte schliesslich zur gewinschten maximalen Relativdurchbiegung des Balkens gemäss Tabelle 12. Eine direkte Kontrolle der Relativdurchbiegungen in Balkenmitte war anhand des Kathodenstrahloszillographen möglich. Mit einem elektronischen Signal konnten zum gewünschten Zeitpunkt mit Hilfe des Servoventils die Del zufuhr zum Schwinger unterbrochen und die Schwingerbewegung innerhalb Sekundenbruchteile gestoppt werden, was ein freies Ausschwingen des Balkens zur Folge hatte. Die aufgezeichneten Beschleunigungen zeigten, dass die Störungen durch den plötzlich gestoppten Schwinger gering waren und sich bereits nach ca. einem Viertel der Dauer eines Schwingzyklus abgebaut hatten. Kurz vor dem Stoppen des Schwingers wurden jeweils das Magnetband sowie der $x$-t-Schreiber gestartet, um die Messignale aufzuzeichnen.

Die statischen Belastungsversuche konnten wie die Ausschwingversuche anhand der Relativdurchbiegung in Bal kenmitte gesteuert werden. Eine stufenweise Variation der Relativdurchbiegung innerhalb der gewünschten maximalen Relativdurchbiegung gemäss Tabelle 12 ermöglichte eine langsame Simulation eines Schwingzyklus. 


\subsection{Versuchsauswertung}

\subsection{1 x-t-Thermo-Schreiber}

Die direkte Aufzeichnung der Messignale durch den x-t-Thermoschreiber (2 Kanäle) ergab kleinformatige Zeichnungen geringer Qualität. Diese Aufzeichnungen dienten zur sofortigen Grobauswertung unmittelbar nach dem Versuch sowie zur Kontrolie der Magnetbandaufnahmen (insbesondere bei der Frequenzermittlung).

\subsubsection{Magnetband}

Die auf dem Magnetband gespeicherten Messignale wurden für jeden Kanal einzeln verlangsamt abgespielt und mit Hilfe eines $x$-t-Schreibers grossformatig aufgezeichnet (Bilder 22a bis 22d). Digitalisierte Signale konnten mit Hil fe einer PCM-Anlage in Analogsignale umgewandelt werden. Auf diese Weise konnte pro Ausschwingversuch und pro Messgeber je eine qualitativ hochwertige Zeichnung des Ausschwingversuchs angefertigt werden. Diese Auf zeichnungen waren die Grundlage für die nachfolgende Auswertung von Hand. Durch Ausmessen der Ausschwingkurven konnten folgende Werte ermittelt werden:

- Rel ativdurchbiegung wrel in Balkenmitte

- Dämpfung (logarithmisches Dekrement $\vartheta$ )

- Eigenfrequenz

- Beschleunigung in Balkenmitte

- relative Stahl- und Betondehnungen.

Die Eigenfrequenz und die Dämpfungswerte wurden in den Teilphasen mit voller Vorspannung (kleine Dämpfung) über ein Intervall von zwanzig, in den Teilphasen mit teilweiser Vorspannung (grössere Dämpfung) über ein Intervall von zehn aufeinanderfolgenden Schwingzyklen ermittelt. Die entsprechenden Werte eines betrachteten Intervalls wurden der Relativdurchbiegung des Schwingzyklus in Intervalimitte zugeordnet. Durch schrittweise Verschiebung des Intervalls um jeweils einen Schwingzyklus liess sich die gesamte Ausschwingkurve abtasten.

Als Kenngrösse für die Dämpfung wurde das nur für ein viskoses Dämpfungsgesetz und für ein lineares verhal ten eines Schwingers gültige logarithmische Dekrement $v$ gewähit. Das effektive Dämpfungsverhal ten genügte diesem Ansatz relativ gut, solange der Versuchsbalken ungerissen war, oder bestehende Risse sich nicht öffneten ( $v g 1$. Abschnitt 1.5). Für Versuchsbalken im gerissenen Zustand hingegen (mit erheblichem Anteil an Reibungsdämpfung, vgl. [2]) ist das logarithmische Dekrement $\theta$ als ein äquivalentes viskoses Dämpfungsmass aufzufassen.

Das logarithmische Dekrement $\vartheta$ ergab sich aus der Beziehung

$$
v=\frac{1}{n} \ln \frac{w_{\mathrm{rel}, \mathrm{m}}}{w_{\mathrm{rel}, \mathrm{m}+n}}
$$

mit:

$\mathrm{n} \quad=$ Anzahl Schwingzyklen im betrachteten Intervall (10 oder 20)

$w_{\text {rel, }}=$ Relativdurchbiegung am Anfang des Intervalis

$w_{r e l, m+n}=$ Relativdurchbiegung am Schl uss des Intervalls

Das logarithmische Dekrement $\vartheta$ lässt sich auf einfache Art in das Dämpfungsmass $\zeta$ umrechnen. Dieses entspricht dem Verhältnis vorhandene Dämpfung/kritische Dämpfung $c_{c r}$ :

$$
\xi=\frac{c}{c_{r}}=\frac{\vartheta}{2 \pi}
$$




\subsubsection{Datenerfassungsanlage}

Die in der Datenerfassungsanlage gespeicherten Messignale der festverdrahteten Messgeber (Kraftmessdosen und induktiver Wegaufnehmer für Relativdurchbiegungen in Balkenmitte) sowie der versetzbaren induktiven Messstange konnten mit Hil fe eines Auswertprogramms ueber einen PDP-11/45 Computer ausgewertet werden. 
Alle gemessenen Versuchswerte wurden in Funktion der im betrachteten Schwingzyklus maximalen Relativdurchbiegung nach unten in Balkenmitte $\mathrm{w}_{\text {rel }}^{+}$angegeben.

Für die Darstellung der Versuchsresultate wurden folgende Konventionen getroffen:

- Die mit $w_{\text {rel }}$ bezeichnete Relativdurchbiegung entspricht der Amplitudenspitze eines betrachteten Schwingzyklus.

- Die Richtung der Relativdurchbiegung wird durch den hochgestellten Index (+) für Relativdurchbiegungen nach unten bzw. (-) für Relativdurchbiegungen nach oben angegeben.

- Die maximalen Relativdurchbiegungen zu Beginn eines Ausschwingversuchs $w_{r e l, m a x}^{+}$(für maximale Relativdurchbiegungen nach unten gemäss Tabelle 12) bzw. $w_{\text {rel, } \max }^{-}$(nach oben) sind in den Bildern mit $u$ resp. mit o markiert.

- Für Phasen wird die Abkürzung P (P1 = Phase 1), für Teilphasen TP (TP 2.3 = Teilphase 2.3) verwendet. Die charakteristischen Eigenschaften der Teilphasen sind den Tabellen 1a bis 1d sowie den Bildern 6a bis 6c zu entnehmen.

- Textstellen, die dem direkten Vergleich von Leichtbeton und Beton dienen, sind am linken Rand mit einem vertikalen Strich markiert.

Falls Resultate aus den Teilphasen 3.2 und 3.4 bzw. 4.2 und 4.4 keine wesentlichen zusätzlichen Erkenntnisse brachten, wurden sie zugunsten der besseren Uebersichtlichkeit der einzelnen Bilder nicht dargestellt.

\subsection{Biegemomente, Spannungen}

Um die gemessenen Versuchswerte (Biegesteifigkeit, Eigenfrequenz, Beschleunigung und Dämpfung) mit der 1 ängs der Balkenachse vorhandenen Biegebeanspruchung in Beziehung bringen zu können, wurden in den Bildern $21 \mathrm{a}$ bis $21 \mathrm{f}$ die Grösse und der Verlauf der Biegemomente bei maximalen Relativdurchbiegungen (zu Beginn eines Ausschwingversuchs gemäss Tabelle 12) aufgezeichnet. Dieselben Bilder zeigen rechts die daraus berechneten Spannungen in Balkenmitte ( $u$-u bei maximalen Relativdurchbiegungen nach unten bzw. 0-o nach oben).

Die Biegemomente $M_{\text {rel }}$ murden mit Hilfe des in der Tabelle 11 dargestellten rechnerischen Zusarmenhangs zwischen Biegemoment, Eigenfrequenz und Relativdurchbiegung und der bei maximalen Relativdurchbiegungen (zu Beginn eines Ausschwingversuchs) gemessenen Eigenfrequenz ermittelt (vgl. Abschnitt 2.3.3). Die Biegemomente $M_{\text {rel }}$ und die zugehörigen Spannungen sind somit als rechnerische Grössen aufzufassen. Sie bilden in dieser Art eine wichtige Grundlage für die Interpretation der in den folgenden Abschnitten besprochenen Versuchsresul tate.

In den Phasen 1 und 2 dürften die rechnerischen Biegemomente $M_{\text {rel }}$ und die zugehörigen Spannungen gut mit den effektiven Werten übereinstimmen, da die rechnerischen Annahmen in Wirklichkeit weitgehend zutrafen. In den Phasen 3 und 4 sind die rechnerischen Biegemomente $M$ rel und die zugehörigen Spannungen als Näherung aufzufassen, da hier die Biegesteifigkeit nach den Gesetzen der elastischen Festigkeitslehre für Biegung mit Normalkraft (Bild 10) sowohl längs der Balkenachse als auch in Funktion der Relativdurchbiegung variiert. In Anbetracht dieser Näherung murden die Biegebeanspruchungen der Phase 3 (nicht injiziert) und der Phase 4 (injiziert) nicht getrennt dargestellt (Bild 21e für den Leichtbetonbalken, Bild 21f für den Betonbalken). 
Die Bilder 2la bis $21 f$ geben folgende Hinweise:

- Bei gleicher Relativdurchbiegung nach unten ergaben sich im ungerissenen Leichtbetonbalken in der Phase 1 37\% kleinere, in der Phase $234 \%$ kleinere Biegemomente $M_{\text {rel }}$ als im ungerissenen Betonbalken. Dieser Unterschied reduzierte sich beim gerissenen, nicht vorgespannten Balken in der Teflphase 2.2 auf $30 \%$ und beim teilweise vorgespannten Balken in der Teilphase 4.5 (100\% Zusatzmassen) auf 9\% (Bilder 21a bis 21f).

- Die Rissöffnungsmomente $M_{\text {ro }}$ wurden in den Teilphasen 1.1 bis 1.3 sowie in den Teilphasen 2.1, 2.3 und 2.4 sowohl beim Leichtbeton- als auch beim Betonbalken nie überschritten. Vor der Teilphase 2.2 war eine Rissbildung somit ausgeschlossen. In den Teilphasen 2.3 und 2.4 konnten sich die in der Teilphase 2.2 gebildeten Risse nicht öffnen (Bilder 21a bis $21 \mathrm{~d}$ ). In den Phasen 3 und 4 wurde das Rissöffnungsmoment in Balkenruhel age erst ab der Teilphase $3.3 \mathrm{bzw} .4 .3$ überschritten (Bilder 21e und $21 \mathrm{f}$ ).

- Die Rissmomente $M_{r}$ wurden in der Teil phase 2.2 bei maximalen Relativdurchbiegungen nach unten bis nahe zum Auflager überschritten, so dass die Versuchsbalken praktisch auf ihrer ganzen Länge reissen konnten (Bilder 21c und 21d, Teilphase 2.2). Bei maximalen Relativdurchbiegungen nach oben wurde das Rissmoment in keiner Phase überschritten. Die Versuchsbalken blieben auf der Balkenoberseite ungerissen.

\subsection{Biegesteifigkeiten}

\subsubsection{Statische Biegesteifigkeiten}

Das Bild 23a zeigt, getrennt für den Leichtbeton- und den Betonbalken, eine Vebersicht der Last-Durchbiegungs-Kurven aus den statischen Bel astungsversuchen während der Phasen 2 bis 4 . Diese wurden jeweils anschliessend an die Ausschwingversuche durchgeführt (keine statischen Belastungsversuche in der Phase 1 ). In den Bildern 23b bis 23d sind, getrennt nach Phasen, die Unterschiede der Last-Durchbiegungs-Kurven von Leichtbeton- und Betonbalken ersichtlich.

Die statische Biegesteifigkeit $B_{\text {stat }}$ liess sich anhand der Last-Durchbiegungs-Kurven berechnen. Dabei wurde unterschieden zwischen einer statischen Tangentensteifigkeit und einer statischen Sekantensteifigkeit.

Die statische Tangentensteifigkeit ist ein Mass für die Steigung der Tangente an die Last-DurchbiegungsKurve in einem Punkt $F_{\text {stat }}\left(w_{\text {rel }}\right)$.

Die statische Sekantensteifigkeit ist ein Mass für die Steigung der Sekante durch zwei Punkte $F_{\text {stat }}^{+}\left(w_{\text {rel }}^{+}\right)$ und $F_{\text {stat }}^{-}\left(w_{\text {rel }}^{-}\right)$der Last-Durchbiegungs-Kurve (vgl. Bild 24a).

Um einen direkten Vergleich mit der dynamischen Biegesteifigkeit (vgl. Abschnitt 4.2.2) zu ermöglichen, wurde die statische Sekantensteifigkeit für den im Ausschwingversuch gemessenen Durchbiegungsbereich $w_{r e l}^{+}$ bis wrel gemäss Tabelle 13 bestimmt. Für die im Bild 12 dargestell te statische Belastungseinrichtung und unter der Annahme konstanter Biegesteifigkeit längs der Balkenachse konnte die statische Sekantensteifigkeit $B_{\text {stat }}$ aus folgender Formel ermittelt werden:

$$
\Delta W=\frac{\Delta F I^{2} s}{24 B_{\text {stat }}}\left(3-4 \frac{s^{2}}{l^{2}}\right)
$$

Mit $1=7.92 \mathrm{~m}, \mathrm{~s}=2.84$ (Abstand der Einzellasten vom Auflager), $\Delta w=w_{\text {rel }}^{+}-w_{\text {rel }}^{-}$(gemäss Tabelle 13) und $\Delta F=F_{\text {stat }}^{+}\left(W_{\text {rel }}^{+}\right)-F_{\text {stat }}^{-}\left(W_{\text {rel }}^{-}\right)$ergibt sich nach Auf 1 ösung nach $B_{\text {stat }}$ :

$$
B_{\text {stat }}=\frac{\Delta F}{\Delta W} 18.45\left[\mathrm{kN} / \mathrm{m}^{3}\right]
$$


Die statische Sekantensteifigkeit $B_{\text {stat }}$ in Funktion der Relativdurchbiegung nach unten ist in den Bildern 24 a bis $24 c$ dargestellt.

Fol gende Feststellungen konnten gemacht werden:

Phase 2

$11=7.92 \mathrm{~m}$, volle Vorspannung und ungerissen in der Teilphase 2.1 , keine Vorspannung in der Teilphase

2.2, volle Vorspannung, aber gerissen in den Teilphasen 2.3 und 2.4)

- Die statische Tangentensteifigkeit war sowohl im voll vorgespannten Zustand als auch im nicht vorgespannten Zustand der Versuchsbalken erwartungsgemäss praktisch unabhängig von der Durchbiegung (1inearer Verlauf der Last-Durchbiegungs-Kurven im Bild 23b, Tei1phasen 2.1 bis 2.4).

- Die statische Sekantensteifigkeit des Leichtbetonbalkens war im voll vorgespannten Zustand ca. $32 \%$ und im nicht vorgespannten Zustand ca. $28 \%$ kleiner als die statische Sekantensteifigkeit des Betonbalkens (Bild 24a, Teilphasen 2.1 und 2.2).

- Die Reduktion der statischen Sekantensteifigkeit infolge Rissbildung betrug beim Leichtbetonbalken $70 \%$, beim Betonbalken 73\% (Bild 24a, Teilphasen 2.1 und 2.2).

- Die statische Sekantensteifigkeit wird im gerissenen Zustand durch die Mitwirkung des Betons zwischen den Rissen erhöht, d.h. der Verbundkoeffizient $\bar{x}=\varepsilon_{s m} / \varepsilon_{s m, m a x}$ ist kleiner als 1.0 (vgl. Abschnitt 2.3.2). Die Tabelle 14 zeigt die gemessenen Dehnungsdifferenzen in Balkenmitte für Relativdurchbiegungen von $w_{\text {rel }}^{+}$bis $w_{\text {rel }}^{-}$gemăss Tabelle 13. Rechnerisch liessen sich Dehnungsdifferenzen aus den in den Bildern 21a bis $21 \mathrm{f}$ dargestell ten Spannungen bestimmen. Da diese Spannungen mit $\bar{k}=1.0$ ermittelt wurden, entsprach (für die Balken im nicht vorgespannten Zustand in der Teilphase 2.2 mit guter Genauigkeit) das Verhăltnis gemessene Stahldehnungsdifferenz/gerechnete Stahldehnungsdifferenz dem Verbundkoeffizienten $\bar{\kappa}$. Für den Leichtbetonbalken liess sich auf diese Weise in der Teilphase 2.2 ein $\bar{k}$ von ca. 0.78 , für den Betonbalken ein $\bar{k}$ von ca. 0.72 berechnen.

Phase 3

$(1=7.92 \mathrm{~m}$, teilweise Vorspannung, Spannglied nicht injiziert, Zusatzmassen)

- Zunehmende Relativdurchbiegungen nach unten führten hier in den teilweise vorgespannten Durchbiegungsbereich und hatten, ausgehend von der Balkenmitte, eine allmähl iche Rissöffnung zur Folge. Die statische Tangentensteifigkeit nahm deshalb erwartungsgemäss mit zunehmender Relativdurchbiegung ab (nicht linearer Verlauf der Last-Durchbiegungs-Kurven in den Bildern 23a und 23c).

- Entgegen den Erwartungen murde in der Teilphase $3.1 \mathrm{im}$ voll vorgespannten Durchbiegungsbereich (Relativduchbiegungen nach oben, überdrückte Risse) eine kleinere státische Tangentensteifigkeit gemessen als in der Teilphase 2.4 (im Bild 23a haben die linear verlaufende Last-Durchbiegungs- Kurve der Teilphase 2.4 und der lineare Bereich der Last-Durchbiegungs-Kurve (1inks unten) der Teilphase 3.1 nicht dieselbe Steigung). Diese Feststellung konnte sowohl beim Leichtbetonbalken als auch beim Betonbalken gemacht werden.

Dieser Steifigkeitsunterschied lässt sich auch anhand der gemessenen Eigenfrequenzen (vgl. Abschnitt 4.3) feststellen. In der Teilphase 3.1 blieb die Eigenfrequenz auch bei kleinen Relativdurchbiegungen kleiner als in der Teilphase 2.4. Eine eindeutige Erklärung für dieses Phänomens koninte nicht gefunden werden. Möglicherweise hatte der Wechsel des Spannungsniveaus, d.h. die Reduktion der zentrischen Vorspannkraft bei Entfernung der zentrischen Spannstäbe, einen entscheidenden Einfluss.

|- Der Leichtbetonbalken und der Betonbalken verhielten sich grundsătzlich gleich (Bild 24b). 


\section{Phase 4}

$(1=7.92 \mathrm{~m}$, teilweise Vorspannung, Spannglied injiziert, Zusatzmassen)

- Im voll vorgespannten Durchbiegungsbereich (Relativdurchbiegungen nach oben) wurde in der Teilphase 4.1 eine nochmals wesentlich kleinere statische Tangentensteifigkeit gemessen als in der Teilphase 3.1 (im Bild 23a haben die linearen Bereiche der Last-Durchbiegungs-Kurven der Teilphasen 3.1 und 4.1 nicht dieselbe Steigung).

Es muss angenommen werden, dass die Bel astungsgeschichte in der Phase 3 (vor allem Auswirkungen der Tei1 phasen 3.3 und 3.5) die statische Tangentensteifigkeit weiter reduziert hat (Verschlechterung der Verbundeigenschaften, Kriecherscheinungen im Beton, zunehmende Mikrorissbildung in der Betondruckzone durch Wechselbeanspruchung, usw.).

- Als Folge dieser Steifigkeitsreduktion wurden im teilweise vorgespannten Durchbiegungsbereich mit hohem Vorspanngrad in den Teilphasen 4.1 und 4.3 trotz Injektion des Hüllrohrs kleinere statische Tangentensteifigkeiten gemessen als in der Tell phase $3.1 \mathrm{bzw} .3 .3$ (nicht injiziert). Erst bei kleinem Vorspanngrad in der Teil phase 4.5 (verglichen mit Tell phase 3.5 ) machte sich die versteifende Wirkung der Injektion bemerkbar.

Die Injektion des Hüllrohrs hatte somit insgesamt einen wesentlich geringeren Einfluss auf die statische Tangentensteifigkeit als die Bel astungsgeschichte.

- Leichtbeton- und Betonbalken verhielten sich grundsätzlich gleich (Bi1d 24c).

\subsubsection{Dynamische Biegesteifigkeiten}

Die dynamische Biegesteifigkeit $B_{\text {dyn }}$ in Funktion der Relativdurchbiegung nach unten kann den Bildern 24a bis $24 \mathrm{C}$ entnommen werden. Sie wurde aus der gemessenen Eigenfrequenz nach folgender Beziehung (vgl. Abschnitt 2.3.3) ermittelt:

$$
B_{d y n}=E I=\frac{4 f^{2}}{\pi^{2}} l^{3}(1 m+2 M)
$$

Wie bereits im Abschnitt 4.2.1 (Phasen 3 und 4) erwähnt, beeinflusste die Bel astungsgeschichte die Biegesteifigkeit stark (Verschlechterung der Verbundeigenschaften, Kriecherscheinungen im Beton, zunehmende Mikrorissbildung in der Betondruckzone durch Wechsselbeanspruchung, usw.). Für einen repräsentativen Vergleich der dynamischen Biegesteifigkeit bei tellweiser Vorspannung mit der dynamischen Biegesteifigkeit bei voller Vorspannung ist der Einfluss der Bel astungsgeschichte möglichst zu eliminieren. Anstelle der dynamischen Biegesteifigkeit $B_{d y n}$ in der voll vorgespannten Teilphase 2.4 wird daher für die Phasen 3 und 4 je ein neuer, die dynamische Biegesteifigkeit bei voller Vorspannung repräsentierender Bezugswert Bdyn,korr definiert.

Die Grundlage dazu bildet die statische Tangentensteifigkeit im linearen Bereich der Last-DurchbiegungsKurve der Teilphase $3.1 \mathrm{bzw}$. der Teilphase 4.1 (Bild 23a: Steigung der Kurven 3.1 und 4.1 im linearen Bereich 1 inks unten). Nach den Beziehungen im Abschnitt 4.2.1 beträgt diese:

- beim Leichtbetonbalken:

Teil phase 3.1: $B_{\text {stat }}=7.35 \times 10^{12}[\mathrm{~N} / \mathrm{mm} 2]$

Teil phase 4.2: $B_{\text {stat }}^{\text {stat }}=6.55 \times 10^{12}[\mathrm{~N} / \mathrm{mm} 2]$

(Al s Vergleich: Teilphase 2.4: $B_{\text {stat }} \approx 9.25 \times 10^{12}[\mathrm{~N} / \mathrm{mm} 2]$ )

- beim Betonbalken:

Teil phase 3.1: $B_{\text {stat }}=11.50 \times 10^{12}[\mathrm{~N} / \mathrm{mm} 2]$

Teil phase 4.1: $B_{\text {stat }}=9.30 \times 10^{12}[\mathrm{~N} / \mathrm{mm} 2]$ 
(Al s Vergleich: Tei1 phase $2.4: B_{\text {stat }}=14.45 \times 10^{12}[\mathrm{~N} / \mathrm{mm} 2]$ )

Durch Multiplikation der dynamischen Biegesteifigkeit der Teilphase 2.4 mit dem Verhältnis der oben ermittel ten statischen Tangentensteifigkeit in der Teilphase $3.1 \mathrm{bzw} .4 .1 \mathrm{zur}$ statischen Tangentensteifigkeit in der Teilphase 2.4 kann näherungsweise auf den vom Einfluss der Belastungsgeschichte befreiten Bezugswert Bdyn,korr geschlossen werden. Es ergeben sich folgende für die volle Vorspannung in den Phasen 3 und 4 repräsentative korrigierte dynamische Biegesteifigkeiten $B_{d y n, k o r r}$ :

- für Leichtbetonbal ken:

Phase 3: $B_{\text {dyn, korr }}=8.3 \times 10^{12} \quad[\mathrm{~N} / \mathrm{mm} 2]$

Phase 4: $B_{\text {dyn, korr }}=7.2 \times 10^{12}[\mathrm{~N} / \mathrm{mm} 2]$

- für Betonbalken:

Phase 3: $B_{\text {dyn, orr }}=12.4 \times 10^{12}[\mathrm{~N} / \mathrm{mm} 2]$
Phase 4: $B_{\text {dyn, korr }}=10.0 \times 10^{12}[\mathrm{~N} / \mathrm{mm} 2]$

Es konnten folgende Feststellungen gemacht werden:

- Die dynamische Biegesteifigkeit lag beim Leichtbeton- und beim Betonbalken generell 5\% bis $10 \%$ über der entsprechenden statischen Sekantensteifigkeit (Bilder 24a bis 24c).

- Die dynamische Biegesteifigkeit nahm mit abnehmender Relativdurchbiegung zu. Die Zunahme war für Relativdurchbiegungen unter 3 bis $5 \mathrm{~mm}$, insbesondere beim Betonbalken, ausgeprägt (Bilder 24a bis 24c). In diesen Bereichen betrug der Unterschied zwischen der dynamischen Biegesteifigkeit und der statischen Biegesteifigkeit $10 \%$ bis $15 \%$.

- Die dynamische Biegesteifigkeit reduzierte sich mit abnehmendem Vorspanngrad in Balkenruhelage in der Phase 4 gegenüber dem jeweiligen Bezugswert für volle Vorspannung $B_{\text {dyn,korr }}$ wie folgt (vgl. Tabelle 15):

Leichtbetonbalken (Phase 4):

$\begin{array}{lll}\kappa_{0}=1.45 & \kappa_{\text {rel, } \max }^{+}=0.69 & \text { Reduktion }=11 \% \\ \kappa_{0}=0.56 & \kappa_{\text {rel, max }}^{+}=0.56 & \text { Reduktion }=15 \% \\ \kappa_{0}=0.48 & \kappa_{\text {rel, max }}^{+}=0.48 & \text { Reduktion }=28 \%\end{array}$

Betonbalken (Phase 4):

$\kappa_{0}=1.17 \quad \kappa_{r e I}^{+}, \max =0.64 \quad$ Reduktion $=16 \%$

$\kappa_{0}=0.80 \quad K_{\text {rel, } \max }^{+}=0.54 \quad$ Reduktion $=31 \%$

$k_{0}=0.61 \quad k_{r e l, \max }^{+}=0.47 \quad$ Reduktion $=45 \%$

Die Reduktion ist im Bild 30a graphisch dargestellt. Die aufgetragenen Punkte geben die dynamische Biegesteifigkeit an, welche bei der Schwingung mit der Maximalbeanspruchung entsprechend $w_{r e l, m a x}^{+}$, d.h. mit dem minimal en Vorspanngrad $\kappa_{r e l, m a x}$, festgestellt wurde. Der jeweils 1 inks vom Punkt dargestellte horizontale Strich gibt an seinem linken Ende (vertikale Markierung) den Vorspanngrad in Balkenruhel age an $\left(\kappa_{0}\right)$.

Die dynamische Biegesteifigkeit reduzierte sich damit wesentlich weniger stark als in der Teilphase 2.2 während der Rissbildung. Es gibt dafür zwei Gründe:

Erstens erhöht das eingelegte Spannglied mit 7 Durchmesser $7 \mathrm{~mm}$ (unter der Annahme, dass dieses lediglich schl aff eingelegt ist) die Biegesteifigkeit des gerissenen Querschnittes in Balkenmitte verglichen mit der Teilphase 2.2 in der Phase 3 (nicht injiziert) um ca. 25\% und in der Phase 4 (injiziert) um ca. 40\% (Leichtbetonbalken und Betonbalken, vgl. Tabelle 8, Querschnittstyp F verglichen mit Querschnittstyp B). 
Zweitens reduziert sich die Biegesteifigkeit beim nicht vorgespannten Balken während der Rissbildung lăngs der ganzen Balkenachse auf etwa den gleichen Wert und bleibt unabhängig von der Relativdurchbiegung, währenddem die Biegesteffigkeit beim teilweise vorgespannten Balken in Balkenmitte bei maximaler Relativdurchbiegung nach unten ein Minimum erreicht und in allen anderen Querschnitts- und Durchbiegungsbereichen höher ist.

\subsection{Eigenfrequenzen}

Die Bilder 25a und 25b zeigen die gemessenen Eigenfrequenzen in Funktion der Relativdurchbiegung nach unten in Balkenmitte. Analog zum Abschnitt 4.2.2 ist auch hier für einen repräsentativen Vergleich der Eigenfrequenzen bei teilweiser Vorspannung mit den Eigenfrequenzen bei voller Vorspannung der Einfluss der Belastungsgeschichte zu eliminieren. Analog zum Abschnitt 4.2.2 wird deshalb für die Eigenfrequenzen in den Phasen 3 und 4 je ein die Eigenfrequenzen bei voller Vorspannung repräsentierender Bezugswert $f_{\text {korr }}$ definiert.

Aus der Beziehung von $B_{d y n}$ und $f$ ergibt sich für $f_{k o r r}$ aus $B_{d y n, k o r r}$ :

- für Leichtbetonbalken:

Phase 3: $f_{\text {korr }}=4.5 \mathrm{~Hz}$

Phase 4: $f_{\text {korr }}=4.19 \mathrm{~Hz}$

- für Betonbalken:

Phase 3: $f_{\text {korr }}=4.98 \mathrm{~Hz}$

Phase 4: $f_{\text {korr }}=4.47 \mathrm{~Hz}$

Das Verhăltnis der Eigenfrequenzen zwischen Leichtbeton- und Betonbalken ist im wesentlichen vom Verhältnis der entsprechenden Leichtbeton- bzw. Betonfestigkeitswerte abhängig (Einfluss des Elastizitätsmoduls auf die Biegesteifigkeit). Bei den nachfolgenden Vergleichen ist somit den Festigkeitswerten gemăss Tabelle 5 Beachtung zu schenken.

Es ist ferner zu beachten, dass im Verlauf des Ausschwingvorgangs in den Phasen 3 und 4 die sich mit abnehmender Relativdurchbiegung verändernde dynamische Biegesteifigkeit die Eigenfrequenz unmittelbar bestimmt. Das heisst, dass die Eigenfrequenz in nachfolgenden Schwingzyklen durch die Eigenfrequenz vorangehender Schwingzyklen nicht beeinflusst wird.

Dies ist aufgrund der folgenden Plausibil itätsbetrachtung leicht einzusehen:

Es gilt die Annahme, dass ein Schwingzykl us seinen Anfang im Bewegungsstillstand, d.h. im Umkehrpunkt der Schwingbewegung, hat. Die Dauer des Schwingzyklus wird durch die am Anfang im Bewegungsstillstand gespeicherte potentielle Energie sowie durch die momentane Biegesteifigkeit, die momentane Balkenmasse und die momentane Balkenspannweite bestinmt. Ob der Balken vorgängig schnell oder langsam in den Bewegungsstil1stand gebracht wurde, hat somit keinen Einfluss auf die nachfolgende Schwingdauer.

Die im Abschnitt 3.3 beschriebene gewähl te Auswertmethode ergibt daher auch für Ausschwingvorgänge mit grösserer oder kleinerer Anfangsamplitude repräsentative Resulate. 
Es konnten folgende Feststellungen gemacht werden:

\section{Allgemein}

Bei beiden Balken war während des Ausschwingversuchs generell eine Abnahme der Eigenfrequenz mit zunehmender Relativdurchbiegung festzustellen. Diese Abnahme war beim Leichtbetonbalken etwas geringer ausgeprägt als beim Betonbalken. Die nachfolgenden Zahlenangaben gelten für eine Relativdurchbiegung nach unten von $1 \mathrm{~mm}$ in der Phase 1, $5 \mathrm{~mm}$ in der Phase 2 und $10 \mathrm{~mm}$ in den Phasen 3 und 4.

\section{Phase 1}

(1 = $5.04 \mathrm{~m}$, volle Vorspannung, zentrische Vorspannkraft)

- Die Eigenfrequenzen waren beim voll vorgespannten Leichtbetonbalken mit $9.8 \mathrm{~Hz}$ trotz geringerer Masse infolge des wesentlich geringeren E-Moduls um $13 \%$ kleiner als beim analogen Betonbalken mit $11.25 \mathrm{~Hz}$ (Bild 25a, Teilphase 1.2).

- Die Grösse der zentrischen Vorspannkraft hatte beim Leichtbetonbalken praktisch keinen Einfluss auf die Eigenfrequenz (Bild 25a, Teilphasen 1.1 bis 1.3). Beim Betonbalken konnte eine geringe Zunahme der Eigenfrequenz mit steigender zentrischer Vorspannkraft festgestellt werden.

- Der Versuchszeitpunkt nach Veränderung der Vorspannkraft hatte (im Gegensatz zur Dämpfung) auf die Eigenfrequenzen praktisch keinen Einfluss, weshalb auf eine graphische Darstellung verzichtet wurde.

\section{Phase 2}

$11=7.92 \mathrm{~m}$, volle Vorspannung und ungerissen in der Teilphase 2.1, keine Vorspannung in der Teilphase 2.2; volle Yorspannung, aber gerissen in den Teil phasen 2.3 und 2.4)

- Die Eigenfrequenzen waren beim voll vorgespannten ungerissenen Leichtbetonbalken mit 5.05 Hz um $11 \%$ kleiner als beim analogen Betonbalken mit $5.65 \mathrm{~Hz}$ (Bild 25a, Teilphase 2.1).

- Durch Vergrösserung der Spannweite von $5.04 \mathrm{~m}$ in der Phase 1 auf 7.92 in der Phase 2 verkleinerte sich die Eigenfrequenz beim Leichtbetonbalken um $49 \%$ und beim Betonbalken un $50 \%$ (Bild 25a, Teilphasen 1.3 und 2.1).

- Die vollständige Weqnahme der Vorspannkraft und die damit verbundene Rissbildung hatten beim Leichtbetonbalken eine Reduktion der Eigenfrequenz von $5.1 \mathrm{~Hz}$ auf $2.85 \mathrm{~Hz}$ um $44 \%$ und beim Betonbalken von $5.65 \mathrm{~Hz}$ auf $3.1 \mathrm{~Hz}$ un $45 \%$ zur Folge (Bild 25a, Teilphasen' 2.1 und 2.2 ).

Schliesslich war die Eigenfrequenz beim nicht vorgespannten gerissenen Leichtbetonbalken um $8 \%$ kleiner als beim analogen Betonbalken.

Vergleich mit der 1. Versuchsreihe dieses Forschungsprojektes: Bei den analogen Versuchsbalken LB 1 und B 1 konnten bei einer Relativdurchbiegung von $5 \mathrm{~mm}$ nach unten für den Leichtbetonbalken und den Betonbalken je etwa dieselbe Eigenfrequenz von $2.8 \mathrm{~Hz}$ gemessen werden (anderes Verhăltnis der Festigkeitswerte von Leichtbeton und Beton).

- Die Einflüsse überdeckter Risse sowie des gekrümmten, nicht injizierten Spannglieds auf die Eigenfrequenz waren sowohl beim Leichtbetonbalken als auch beim Betonbalken erwartungsgemäss klein Bild 25a, Teil phasen 2.1, 2.3 und 2.4).

- Die Einflüsse überdeckter Risse sowie des gekrümmten, nicht injizierten Spanngl ieds auf die Eigenfrequenz waren sowohl beim Leichtbetonbalken als auch beim Betonbalken erwartungsgemäss klein (Bild 25a, Teilphasen 2.1, 2.3 und 2.4). 
Phase 3

$(1=7.92 \mathrm{~m}$, teilweise Vorspannung, Spannglied nicht injiziert, Zusatzmassen)

- Die aufgebrachten Zusatzmassen verkleinerten die Eigenfrequenz aus zweierlei Gründen. Einerseits wurde dadurch der Vorspanngrad in Balkenruhelage und damit die Biegesteifigkeit reduziert, andererseits erhöhte sich gleichzeitig die mitschwingende Masse. Durch das Aufbringen von $100 \%$ Zusatzmassen (Bild 7) reduzierte sich die Eigenfrequenz des Leichtbetonbalkens von $4.32 \mathrm{~Hz}$ auf $2.42 \mathrm{~Hz}$ um $44 \%$ und diejenige des Betonbalkens von $4.49 \mathrm{~Hz}$ auf $2.38 \mathrm{~Hz}$ um $47 \%$ (Bild 25b, Teilphasen 3.1 und 3.5).

- Die Abhängigkeit der Eigenfrequenz vom Vorspanngrad allein ist in der Tabelle 15 dargestellt. Als Bezugsgrösse für volle Vorspannung diente dabei die korrigierte Eigenfrequenz $f_{\text {korr }}$ in der Phase 3 . Um den Einfluss des Vorspanngrades allein auf die Eigenfrequenz ermitteln zu können, mussten die gemessenen Werte mit Hilfe der Beziehungen im Abschnitt 2.3.3 auf eine einheitl iche Balkenmasse ungerechnet werden.

Die Reduktion der Eigenfrequenz bei der Verkleinerung des Vorspanngrades in Balkenruhelage war beim Leichtbetonbalken geringer als beim Betonbalken.

Bei ungefähr gleichem Yorspanngrad und ungefähr gleicher Gesamtmasse lagen die Eigenfrequenzen beim Leichtbetonbalken um 16\% (LB in der Teilphase 3.2 und $B$ in der Teilphase $3.1, K_{0}=1.16$ und $\mathrm{K}_{\text {rel, }}^{+}$, 0,63 ) bzw. um $10 \%$ (LB in der Teilphase 3.4 und $B$ in der Teilphase 3.3, $\kappa_{0}=0.8$ und $\kappa_{\text {rel, }}^{+}$max $=0.53$ ) tiefer als beim Betonbalken (Bild 25b, Tabelle 3 ).

\section{Phase 4}

$(1=7.92 \mathrm{~m}$, teilweise Vorspannung, Spannglied injiziert, Zusatzmassen)

- Entgegen den Erwartungen lagen die Eigenfrequenzen nach der Injektion des Hüllrohrs in der Teilphase 4.1 nicht höher, sondern beim Leichtbetonbalken um 7\% und beim Betonbalken um $8 \%$ tiefer als in der Jeil phase 3.1 (Bild 25b).

Damit wurde die im Abschnitt 4.2.1 in der Phase 4 erwähnte Steifigkeitsreduktion infolge der Belastungsgeschichte (vor allem Auswirkungen der Tell phasen 3.3 und 3.5) bestätigt.

- Die Abhängigkeit der Eigenfrequenz vom Vorspanngrad allein ist in der Tabelle 15 und im Bild 30b dargestellt. Als Bezugsgrösse für volle Vorspannung diente dabei die korrigierte Eigenfrequenz $f_{\text {korr }}$ der Phase 4. Die aufgetragenen Punkte im Bild $30 \mathrm{~b}$ geben die auf eine einheitliche Balkenmasse umgerechnete Frequenz $\bar{f}$ an, die bei der Schwingung mit der Maximalbeanspruchung entsprechend $w_{\text {rel, max }}^{+}$d.h. mit dem minimal en Vorspanngrad $\kappa_{\text {rel, }}^{+}$fexax festgestel1t wurde. Der jeweils 1 inks vom Punkt angebrachte Strich gibt an seinem linken Ende (vertikale Markierung) den Vorspanngrad $k_{j}$ in Balkenruhel age an.

Die Eigenfrequenz reduzierte sich mit abnehmendem Vorspanngrad in Balkenruhelage in der Phase 4 bezogen auf den jeweiligen Bezugswert für volle Vorspannung, $f_{\text {korr }}$, wie folgt (Einfluss der Zusatzmassen el iminiert):

\begin{tabular}{|c|c|c|c|}
\hline$k_{0}=1.45$ & $\mathrm{~K}_{\mathrm{rel}, \max }^{+}$ & $=0.69$ & Reduktion $=6 \%$ \\
\hline$=0.91$ & & $=0.56$ & $=8 \%$ \\
\hline$=0.48$ & & $=0.48$ & $=16 \%$ \\
\hline
\end{tabular}

\begin{tabular}{|c|c|c|c|}
\hline$\kappa_{0}=1.17$ & $K_{\text {rel, } \max }^{+}$ & $=0.64$ & Reduktion $=9 \%$ \\
\hline$=0.80$ & & $=0.54$ & $=17 \%$ \\
\hline$=0.61$ & & $=0.47$ & $=26 \%$ \\
\hline
\end{tabular}


- Im übrigen verhielten sich die Eigenfrequenzen der injizierten Versuchsbalken in der Phase 4 gleich wie beim nicht injizierten Versuchsbalken in der Phase 3 (Bild 25b).

\subsection{Beschleunigungen}

Im Bild 26 sind die in Balkenmitte gemessenen maximal en Beschleunigungen in Funktion der Relativdurchbiegung nach unten dargestellt. Die Beschleunigung a wird im wesentlichen durch die Relativdurchbiegung w re sowie durch die Masse und die Steifigkeit bestimmt. Für den Einmassenschwinger mit sinusförmigem Schwingverlauf gilt:

$$
a=w_{\text {rel }} \frac{k}{m}
$$

Darin sind $k$ die Federsteifigkeit und $m$ die Masse des Einmassenschwingers.

Dies ergibt für den Versuchsbalken unter der Annahme konstanter Biegesteifigkeit lăngs der Balkenachse $E I=B=$ konstant (vgl. auch Abschnitt 2.3.3):

$$
a_{\text {rechn }}=w_{r e l} \frac{\pi^{4}}{1^{3}}\left(\frac{B}{1 m+2 M}\right)
$$

Darin sind $m$ die Masse pro $m^{\prime}$ Balkenlänge (inkl. Zusatzmassen) und $M$ die Masse des servohydraulischen Schwingers.

Im Bild 27 ist für die Phasen 2 bis 4 das Verhältnis Rechnerische Beschleunigung a rechn (mit dynamischer Biegesteifigkeit aus dem Bild 24 längs der Balkenachse als konstant angenommen)/Effektiv in der Balkenmitte gemessene Beschleunigung $a_{\text {ef }}^{+}$für Relativdurchbiegungen nach unten dargestellt.

Es konnten fol gende Feststel lungen gemacht werden:

- Das Verhältnis $a_{\text {rechn }} / a_{\text {ef }}^{+}$betrug in den Phasen 1 und 2 nahezu 1.0. Da die Rechenannahmen gut mit der Wirklichkeit übereinstimmten (konstante Biegesteifigkeit längs der Balkenachse; Biegesteifigkeit praktisch unabhängig von der Relativdurchbiegung), entsprach dies den Erwartungen (Bild 27, Teilphasen 2.1 bis 2.4).

- Das Verhältnis a ${ }_{\text {rechn }} / a_{\text {ef }}^{+}$bei Relativdurchbiegungen nach unten stieg in den Phasen 3 und 4 bis auf 1.25 (Bild 27, Teil phasen 3.1 bis 4.5 ). Im Gegensatz dazu war das Verhältnis a rechn $/ a_{\text {ef }}^{-}$bei Relativdurchbiegungen nach oben deutlich kleiner als 1.0 (nicht dargestellt). Dies kommt daher, dass im Durchbiegungsbereich unterhalb der Balkenruhelage der Balken weicher war. Die Balkenmasse wird auf einer grösseren Durchbiegungsstrecke verzögert bzw. beschleunigt als im Beanspruchungsbereich oberhalb der Balkenachse.

- Das Verhăltnis $a_{\text {rechn }} / a_{\text {ef }}^{+}$lag in den Phasen 3 und 4 nur dann etwa bei 1.0, wenn sich die Relativbeanspruchungen grösstenteils im Bereich der vollen Vorspannung (Leichtbeton Teilphasen 3.1 und 4.1) oder grösstenteils im Bereich der teilweisen Vorspannung (Beton Teilphasen 3.5 und 4.5) bewegten (Bild 27), d.h., wenn sich die statische Tangentensteifigkeit im Durchbiegungsbereich eines Schwingzyklus nur unwesentl ich veränderte. 
Die Bilder 28a bis 28d zeigen den aus den Ausschwingversuchen ermittelten Verlauf des logarithmischen Dekrementes $\diamond \mathrm{bzw}$. des Dämpfungsmasses $\xi$ in Funktion der Relativdurchbiegung nach unten in Balkenmitte. Definitionen und Berechnungshinweise $z u$ v und $\xi$ sind im Abschnitt 3.3 gegeben.

Es ist zu beachten, dass die Dämpfungswerte bei nicht vorgespannten Balken gemăss [1] auf etwa die Hălfte reduziert werden, falls der Gehalt an schlaffer Bewehrung etwa verdreifacht wird. Dasselbe dürfte auch für teilweise vorgespannte Balken zutreffen.

Es konnten folgende Feststellungen gemacht werden:

Phase 1

$(1=5.04 \mathrm{~m}$, volle Vorspannung, zentrische Vorspannkraft $)$

- Die Dämpfung des voll vorgespannten Leichtbeton- und des Betonbalkens reduzierte sich mit zunehmender Zei tspanne zwischen Veränderung der Vorspannkraft und Versuchszeitpunkt. Die Dămpfungswerte erreichten ca. 48 Stunden nach Veränderung der Vorspannkraft asymptotisch den Tiefstwert (Bi1d 29).

- Der Tiefstwert der Dämpfung des voll vorgespannten Leichtbetonbalkens war mit $\xi=0.4 \%$ un $44 \%$ geringer als derjenige des analogen Betonbalkens mit $\xi=0.7 \%$ (Bild $28 \mathrm{~b}$, Teilphase $1.3, t=48 \mathrm{~h}$ ). Die Reduktion der Dämpfung innert 48 Stunden betrug beim Leichtbetonbalken ca. $27 \%$ und beim Betonbalken ca. $20 \%\left(w_{\text {rel }}^{+}=3.5 \mathrm{~mm}\right)$.

- Die Grösse der Relativdurchbiegung hatte sowohl beim Leichtbeton- als auch beim Betonbalken praktisch keinen Einfluss auf die Dämpfung (Bild 28b, Tellphasen 1.1 bis 1.3 ).

- Die Höhe der zentrischen Vorspannkraft hatte sowohi beim Leichtbeton- als auch beim Betonbalken nur einen geringen Einfluss auf die Dămpfung (Bild 28b, Teilphasen 1.1 bis $1.3, t=1 \mathrm{~h}$ ). Bei grösserer zentrischer Vorspannung ergab sich generell eine etwas grössere Dämpfung. Es darf vermutet werden, dass sich für einen Versuchszeitpunkt $t=\infty$ nach Veränderung der Vorspannkraft etwa dieselben Dämpfungswerte, praktisch unabhängig von der Grösse der Vorspannkraft, ergeben.

Phase 2 (voll vorgespannt):

$(1=7.92 \mathrm{~m}$, volle Vorspannung und ungerissen in der Teil phase 2.1, volle Vorspannung, jedoch gerisssen in den Teil phasen 2.3 und 2.41

- Trotz Halbierung der Eigenfrequenz gegenüber Phase 1 durch Vergrösserung der Spannweite, veränderte sich die Dämpfung nicht wesentlich (Bild 28a, Teilphasen 1.3 und 2.1, $t=1 \mathrm{~h}$ ). Die Dämpfung in voll vorgespannten Leichtbeton- und Betonbalken war somit praktisch unabhängig von der Eigenfrequenz und betrug eine Stunde nach Veränderung der Vorspannkraft beim Leichtbetonbalken ca. $\xi=0.5 \%$ und beim Betonbalken ca. $\xi=0.8 \%$.

- Im vollständig gerissenen, jedoch wieder voll vorgespannten Zustand (Risse überdrückt) erhöhte sich die Dämpfung verglichen mit dem voll vórgespannten ungerissenen Zustand beim Leichtbetonbalken um ca. $35 \%$, beim Betonbalken um ca. $31 \%$ (Bild 28b, Teilphasen 2.1 und 2.3). Eine eindeutige Erk1ärung hiefür ist schwierig zu geben, doch es handelt sich vermutlich um eine Energiedissipation in den überdrückten Rissen.

- Der Wechsel von zwei geraden exzentrischen Spannstäben zum gekrümmten Spannglied hatte keinen Einfl uss auf das Dămpfungsverhal ten (Bild 28b, Teilphasen 2.3 und 2.4). In der Teil phase 2.4 fanden so- 
mit zwischen dem Hüllrohr und dem Spannstahl (Paralleldrahtbündel) keine merklichen Relativverschiebungen statt (ausreichende Haftreibung dank Hüllrohrkrümmung).

Phase 2 (ohne Vorspannung):

$(1=7.92 \mathrm{~m}$, Spannstäbe entfernt, gerissen, Teilphase 2.2)

- Bei gleicher Relativdurchbiegung war die Dämpfung beim Leichtbetonbalken $28 \%$ bis $32 \%$ kleiner als beim Betonbalken (Bild 28c, Teilphase 2.2). Die Dämpfungsmass varijerte beim Leichtbetonbalken zwischen etwa $\xi=2 \%$ und $3.5 \%$ und beim Betonbalken zwi schen etwa $3.5 \%$ und $4.5 \%$.

- Ein Vergleich mit den gleich bewehrten Versuchsbalken LB 1 und B 1 aus der 1 . Versuchsreihe zeigt eine befriedigende Uebereinstimmung der gemessenen Dămpfungswerte beim Leichtbetonbalken und eine gute Uebereinstimung beim Betonbalken (Bild 28c, Teilphase 2.2, LB 1, B 1).

Phase 3:

$(1=7.92 \mathrm{~m}$, teilweise Vorspannung, Spannglied nicht injiziert, Zusatzmassen)

- Bei gleichem Vorspanngrad (Zusatzmassen verschieden) war die Dämpfung beim Leichtbetonbalken ca. 30\% kleiner als beim Betonbalken (Tabelle 3, LB Teilphase 3.2 und B Teilphase 3.1, LB Teilphase 3.4 und B Teilphase 3.3).

- Bei gleich viel Zusatzmassen war die Dämpfung beim Leichtbetonbalken um ca. $40 \%$ kleiner als beim Betonbalken (Bild 28c, Teilphasen 3.1 bis 3.5 ).

- Mit zunehmender Relativdurchbiegung nahm die Dămpfung analog wie beim nicht vorgespannten Balken stark ab und strebte asymptotisch einem verhältni smässig tiefen Endwert zu (Bild $28 \mathrm{c}$, Teilphasen 3.1 bis 3.5). Das Dämpfungsmass varifierte dabei beim Leichtbetonbal ken zwischen etwa $\xi=2.5 \%$ und $1.0 \%$ und beim Betonbalken zwi schen etwa $4.0 \%$ und $1.5 \%$.

- Die Dämpfung erhöhte sich im Bereich grösserer Relativdurchbiegungen durch Reduktion des Vorspanngrades in Balkenruhel age $\kappa_{0}$ von 1.45 auf 0.67 beim Leichtbetonbalken und um ca. $30 \%$ und bei Reduktion von $k_{0}$ von 1.17 auf 0.61 beim Betonbalken um ca. $40 \%$ (Bild 28c, Teilphasen 3.1 und 3.5).

- Im Zusammenhang mit den sich öffnenden Rissen entstanden erhebliche Relativverschiebungen zwischen dem Spannstahl und dem gekrïmmten Hüllrohr. Diese konnten während des Ausschwingens akustisch wahrgenommen werden.

\section{Phase 4:}

( 1 = $7.92 \mathrm{~m}$, teilweise Vorspannung, Spannglied injiziert, Zusatzmassen)

- Die Injektion des Hüllrohrs führte sowohl beim Leichtbetonbalken als auch beim Betonbalken bei hohem Vorspanngrad (Teilphase 4.1) zu einer teilweisen Erhöhung, bei kleinem Vorspanngrad (Teilphase 4.5) zu einer leichten Reduktion der Dămpfung (Bild 28a, Teil phasen 3.1 und 4.1, Teil phasen 3.5 und 4.5).

- Mit zunehmender Relativdurchbiegung nahm die Dämpfung ähnlich wie bei den nicht vorgespannten Bal ken und analog zu der Phase 3 ab und strebte asymptotisch einem verhältnismässig tiefen Endwert zu (Bild 28d, Teil phasen 4.1 bis 4.5). Das Dämpfungsmass variierte dabei etwa zwischen den 'gleichen Grenzen wie in der Phase 3.

- Eine eindeutige Abhängigkeit der Dämpfung vom Vorspanngrad in Balkenruhelage $\kappa_{0} 1$ iess sich in Phase, 4 nicht feststellen (Bild 28d). Grundsătzlich liegt die Dämpfung bef teilweiser Vorspannung zwischen den beiden Extremwerten des voll vorgespannten Balkens und des nicht vorgespannten Balkens (schema- 
tisch dargestellt im Bild 30c; die aufgetragenen vollen Punkte geben die Dämpfung an, welche bei der Schwingung mit Maximalbeanspruchung entsprechend $w_{\text {rel, max }}^{+}$, d.h. mit dem minimalen Vorspanngrad $\kappa_{\text {rel, max }}^{+}$, festgestellt wurde. Die Pfeile geben an, in welcher Richtung sich die Dämpfung mit abnehmender Rel ativdurchbiegung veränderte.) 
Neue Materialien wie Leichtbeton und neue Technologien wie Vorspannung ermöglichen den Bau ausgesprochen schlanker Bauwerke, die leicht in Schwingungen mit grossen Amplituden versetzt werden können. Im Rahmen des Forschungsprojektes "Dynamisches Verhalten von Leichtbeton- und Betonbalken" wurden am Institut für Baustatik und Konstruktion der Eidgenössischen Technischen Hochschule Zürich (ETH Zürich) an je einem vorgespannten Leichtbeton- und Betonbaiken Versuche durchgeführt. Diese Versuche ergänzen eine erste im Jahr 1979 abgeschlossene Versuchsreihe an nur schlaff bewehrten Leichtbeton- und Betonbalken.

Die im vorliegenden Bericht beschriebenen Versuche hatten zum Ziel, den Einfluss des Vorspanngrades, der Rissbildung und der Beanspruchungshöhe auf die dynamischen Eigenschaften wie Biegesteifigkeit, Eigenfrequenz und Dämpfungsverhalten von voll und teilweise vorgespannten Leichtbeton- und Betonbalken im quasielastischen Beanspruchungsbereich zu untersuchen. Ferner sollte ein Vergleich zwischen Leichtbetonbalken und analogen Betonbalken gemacht werden.

Die untersuchten Leichtbeton- und Betonbalken wiesen eine Länge von $8.4 \mathrm{~m}$ und einen Rechteckquerschnitt von $0.4 \mathrm{~m}$ Breite und $0.24 \mathrm{~m}$ Höhe auf. Die dynamischen Eigenschaften der als einfache Balken gelagerten und vorspannbaren Träger wurden anhand einfacher Ausschwingversuche ermittelt. Die Anregung erfolgte mit einem servohydraulisch gesteuerten Schwinger in Balkenmitte. Mit Hilfe einer statischen Belastungseinrichtung konnte zudem die Biegesteifigkeit bei statischer Relativbeanspruchung gemessen werden.

Das Versuchsprogramm umfasste bei beiden Balken vier Phasen:

In der Phase 1 wurden die Balken mit einer reduzierten Spannweite von $1=5.04 \mathrm{~m}$ und auskragenden Enden von je $1.68 \mathrm{~m}$ im voll vorgespannten Zustand gepriff. Mit Hilfe von sechs geraden, nicht injizierbaren Spannstäben konnte eine veränderbare zentrische Vorspannkraft aufgebracht werden.

In der Phase 2 wurde die Spannweite auf $7.92 \mathrm{~m}$ erhöht. Volle Vorspannung durch gerade Spannstäbe in der ersten Teilphase, keine Vorspannung (Rissbildung) nach Entfernung der Spannstäbe in der zweiten Teilphase und wiederum volle Vorspannung (Risse unterdrückt) in der dritten Teilphase ergaben Grenzwerte für das dynamische Verhalten. In der vierten Teilphase wurden die geraden Spannstäbe teilweise durch eine parabelförmig gekrümmtes Spannglied ersetzt, um den Einfluss der Spanngliedführung zu untersuchen.

In der Phase 3 konnten durch schrittweises Aufbringen von Zusatzmassen der Vorspanngrad in Balkenruhelage reduziert und die Balken im teilweise vorgespannten Zustand geprüft werden. Das gekrümmte Spannglied war in dieser Phase nicht injiziert.

Die Phase 4 verlief analog zur Phase 3, jedoch mit injiziertem gekrümmtem Spannglied.

Im folgenden werden die Resultate zusammengefasst. Der Vorspanngrad $k$ gilt jeweils für die Balkenmitte. $\kappa_{0}$ ist der Vorspanngrad in Balkenruhelage, $\kappa_{r e l, \max }^{+}$ist der Vorspanngrad bei maximalen Relativdurchbiegungen nach unten. Dem direkten Vergleich zwischen Leichtbeton und Beton dienende Textteile sind links am Rand mit einem vertikalen Strich versehen.

\section{Biegesteifigkeiten}

- Die Reduktion der dynamischen Biegesteifigkeit bei teilweiser Vorspannung gegenüber dem voll vorgespannten Zustand betrug: Leichtbetonbalken (Phase 4):

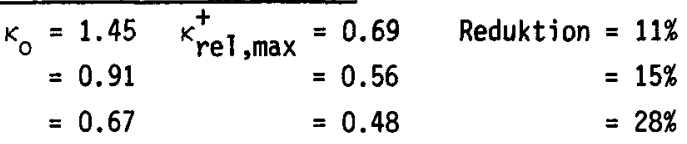

\section{Betonbalken (Phase 4):}

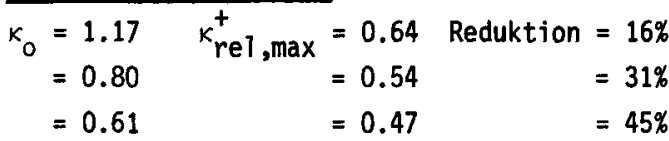

Die Reduktion war damit beim Leichtbetonbalken erheblich geringer als beim Betonbalken.

- Die statische Biegesteifigkeit (Sekantensteifigkeit) lag für die Leichtbeton- und Betonbalken im untersuchten Frequenzbereich ( $f=2$ bis $6 \mathrm{~Hz}$ ) bei grossen Relativdurchbiegungen ca. 5\% bis 10\% tiefer als die 
dynamische Biegesteifigkeit. Bei kleinen Relativdurchbiegungen erhöhte sich der Unterschied auf $10 \%$ bis $15 \%$.

- Die dynamische und die statische Biegesteifigkeit waren beim Leichtbetonbalken im voll vorgespannten Zustand ca. $32 \%$, im nicht vorgespannten Zustand ca. $28 \%$ kleiner als diejenigen des analogen Betonbalkens.

- Die Belastungsgeschichte hatte einen grossen Einfluss auf die Biegesteifigkeiten.

\section{Eigenfrequenzen}

- Die Eigenfrequenzen variierten beim Leichtbetonbalken zwischen $9.8 \mathrm{~Hz}$ (volle Vorspannung, Spannweite $1=5.04 \mathrm{~m}$ ) und $2.4 \mathrm{~Hz}$ (teilweise Vorspannung, Zusatzmassen, Spannweite $1=7.72 \mathrm{~m}$ ), beim Betonbalken zwischen $11.25 \mathrm{~Hz}$ und $2.4 \mathrm{~Hz}$.

- Die Reduktion der Eigenfrequenzen bei teilweiser Vorspannung betrug gegenüber dem voll vorgespannten Zustand (Einfluss der Zusatzmassen eliminiert):

Leichtbetonbalken (Phase 4):

$\begin{aligned} \kappa_{0} & =1.45 & \kappa_{\text {rel, } \max }^{+} & =0.69 & \text { Reduktion } & =6 \% \\ & =0.91 & & =0.56 & & =8 \% \\ & =0.67 & & =0.48 & & =16 \%\end{aligned}$
Betonbalken (Phase 4):

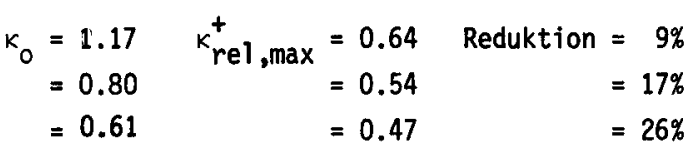

Die Reduktion war damit beim Leichtbetonbalken wesentlich geringer als beim Betonbalken.

- Die Belastungsgeschichte hatte einen grossen Einfluss auf die Eigenfrequenzen.

Dämpfung

Im voll vorgespannten Zustand:

- Die Dämpfung reduzierte sich mit zunehmender Zeitspanne zwischen Veränderung der Vorspannkraft und Versuchszeitpunkt. Sie erreichte ca. 48 Stunden nach Veränderung der Vorspannkraft etwa asymptotisch den Tiefstwert und lag beim Leichtbetonbalken $27 \%$ und beim Betonbalken $20 \%$ tiefer als 1 Stunde nach Veränderung der Vorspannkraft. Der erreichte Tiefstwert des Dämpfungsmasses betrug beim Leichtbetonbalken $\xi=c / c_{c r}=0.4 \%$, beim Betonbalken $\xi=c / c c r=0.7 \%$.

Die Dämpfung des voll vorgespannten Leichtbetonbalkens war damit $44 \%$ geringer als beim analogen Betonbalken.

- Die Dämpfung war praktisch unabhängig von der Relativdurchbiegung und der Grösse der zentrischen Vorspannkraft, d.h. praktisch unabhängig von der Beanspruchungshöhe (innerhalb des quasi-elastischen Bereichs).

- Die Dämpfung war unabhängig von der Frequenz.

\section{Im teilweise vorgespannten Zustand:}

Es ist zu beachten, dass die Dämpfungswerte bei nicht vorgespannten Balken gemäss [1] auf etwa die Hälfte reduziert werden, falls der schlaffe Bewehrungsgehalt verdreifacht wird. Dasselbe dürfte für teilweise vorgespannten Balken zutreffen.

- Die Dämpfung nahm mit zunehmender Relativbeanspruchung analog den nicht vorgespannten Balken stark ab und strebte asymptotisch einem tiefen Endwert zu. Das Dämpfungsmass vari ierte dabei beim Leichtbetonbalken zwischen $\xi=2.5$ und $\xi=1.0 \%$ und beim Betonbalken zwischen $\xi=4 \%$ und $\xi=1.5 \%$. Die Dämpfung erhöhte sich somit bei teilweiser Vorspannung (nach vollständiger Rissbildung) gegenüber voller Vorspannung um rund das Sechsfache bei kleiner Relativbeanspruchung und um rund das Zweifache bei grosser Relativbeanspruchung. Die Grössenordnung der Dämpfung wurde durch die Spanngliedinjektion nur unwesentlich beeinflusst.

- Die Dämpfung erhöhte sich bei Balken mit nicht injiziertem Spannglied durch Reduktion des Vorspanngrades in Balkenruhelage $\kappa_{0}$ von 1.45 auf 0.67 beim Leichtbetonbalken um ca. $30 \%$ und bei Reduktion von $\kappa_{0}$ um 1.17 auf 0.61 beim Betonbalken um ca. $40 \%$.

- Die Dämpfung war beim teilweise vorgespannten Leichtbetonbalken bei gleichem Vorspanngrad in Balkenruhelage ca. $30 \%$ kleiner als beim Betonbalken. 


\section{Im nicht vorgespannten Zustand:}

Es ist zu beachten, dass die Dämpfungswerte für höhere Bewehrungsgehalte gemäss [1] erheblich niedriger ausfallen können als die hier angegebenen Werte (vgl. Abschnitt 1.4).

- Das Dämpfungsmass vari ierte beim Leichtbetonbalken zwischen $\xi=3.5 \%$ und $\xi=2.0 \%$, beim Betonbalken $z$ wischen $\xi=4.5 \%$ und $\xi=3.5 \%$.

- Die Uebereinstimmung mit den Balken mit gleichem schlaffem Bewehrungsgehalt der 1. Versuchsreihe dieses Forschungsprojektes (LB 1 und B1 in [1]) war gut. 
De nouvaux matériaux corme le béton léger et de nouvelles techniques corme la précontrainte permettent 1a construction d'ouvrages extraordinairement élancês, qui peuvent être facilement mis en vibration avec de grandes amplitudes. Dans le cadre du projet de recherche "Comportement dynamique de poutres en béton léger et en béton normal" des essais ont été effectués à l'institut de statique et construction de 1 'Ecole Polytechnique fédérale de Zürich. Ces essais portẻrent sur deux poutres précontraintes, l'une avec du béton léger et l'autre avec du béton normal; ils complètent une premiēre série d'essais, terminée en 1979, sur des poutres en béton léger et en bêton noma1, armées seulement d'armatures passives.

Les essais dẽcrits dans le présent rapport avaient pour but d'étudier l'influence du degré de précontrainte, de la formation des fissures et de la hauteur des sollicitation sur les propriétés dynamique tel que la rigidité à la flexion, les fréquences propres et le comportement de l'amortissement des poutres en béton léger ou en béton normal complètement ou partiellement précontraintes dans le domaine des sollicitations quasi-êlastiques. De plus une comparaison entre des poutres en bēton léger et en béton normal est faite.

Les poutres étudiées en bēton léger ou en béton normal avaient une longueur de $8.4 \mathrm{~m}$ et une section rectangulaire de $0.4 \mathrm{~m}$ de large et de $0.24 \mathrm{~m}$ de haut. Les propriétés dynamiques des poutres ont êté déterminées à l'aide d'essais d'oscillations simples. Les poutres êtaient prēcontraintes et les conditjons d'appuis étaient celles d'une poutre simple. Les sollicitations étaient produites à l'aide d'une masse vibrante, asservie hydrauliquement, située au milieu des poutres. La rigidité à la flexion pouvait être en outre mesurée a l'aide d'une installation de chargement statique pour des sollicitation relatives statiques.

Le programme d'essais comportait pour les deux poutres quatre phases:

- Dans la phase I, les poutres étaient testees avec une portée réduite de $1=5.04 \mathrm{~m}$ et un port-à-faux de $1.68 \mathrm{~m}$, dans un état de précontrainte totale. Une force de précontrainte centrée, modifiable, pouvait être exercée à l'aide de six barres d'aciers qui n'étaient pas injectables.

- Dans le phase Il, la portée était augmentée à $7.92 \mathrm{~m}$. Les valeurs limités du comportement dynamique étaient mesurées à partir des trois tests suivants: dans le permier test précontrainte totale à l'aide des barres d'acier droite, dans le deuxième test pas de prēcontrainte (formation de fissures) après enlèvement des barres d'acier, et dans le troisième test à nouveau précontrainte totale (compression des fissures). Dans le quatrième test de la phase II, les barres d'acier droites furent partiellement remplacées par un câble de précontrainte parabolique, pour étudier l'influence de la forme de la précontrainte.

- Dans la phase III, le degré de précontrainte de la poutre dans la position d'équilibre a pu être réduit en rajoutant par étape des masses supplémentaires, pour que les poutres soient testées dans un état de précontrainte n'était pas injecté.

- La phase IV se déroula d'une façon analogue à la phase III, mais le câble de précontrainte parabolique êtait injectē.

Les résultats des essais sont résumés ci-dessous. Le degré de précontrainte $\kappa$ est valable pour le milieu de la poutre. $\kappa_{0}$ est le degré de précontrainte dans la position au repos. $\kappa_{r e l \text {, max }}^{+}$est le degré de précontrainte pour une déflexion maximale relative en direction du bas.

Les parties du texte comparant directement le béton léger avec le béton normal sont indiquées par une ligne verticale dans la marge de gauche.

\section{Rigidité à la flexion}

- La réduction de la rigidité dynamique à la flexion pour un état de précontrainte partielle par rapport à un état de précontrainte totale se montait: 
Poutres en bëton léger (Phase 4):

$$
\begin{aligned}
& \kappa_{0}=1.45 \quad k_{r e l, \max }^{+}=0.69 \text { Réduction }=11 \% \\
& =0.91=0.56=15 \% \\
& =0.67=0.48=28 \%
\end{aligned}
$$

\section{Poutres en béton normal (Phase 4):}

$\begin{aligned} K_{0} & =1.17 & k_{\text {re1, max }}^{+} & =0.64 & \text { Réduction } & =16 \% \\ & =0.80 & & =0.54 & & =31 \% \\ & =0.61 & & =0.47 & & =45 \%\end{aligned}$

La rêduction était ainsi bien inférieure pour le béton léger que pour le béton normal.

- La rigidité à la flexion statique (rigidité sécante) est inférieure à la rigidité dynamique, pour des poutres en béton léger et en bêton normal dans le domaine de fréquences étudiées (f $=2$ a $6 \mathrm{~Hz}$ ) pour des déflexions relatives d'environ 5 à $10 \%$. Pour de petites déflextions la différence s'êlève à $10 \%$, jusqu'à $15 \%$.

|- La rigidité à la flexion dynamique et statique de la poutre en béton léger étaient par rapport à la poutre en bẻton normal d'environ $32 \%$ inférieure dans un état de précontrainte totale et d'environ $28 \%$ inférieures dans un état non-précontraint.

- L'histoire de la charge avait une grande influence sur la rigiditê à la flexion.

\section{Fréquences propres}

- Les fréquences propres varièrent pour les poutres en béton léger entre $9.8 \mathrm{~Hz}$ (prêcontrainte totale, portée $1=5.04 \mathrm{~m}$ ) et $2.4 \mathrm{~Hz}$ (précontrainte partielle, masses additionnelles, portée $1=7.72 \mathrm{~m}$ ) pour les poutres en béton normal entre $11.25 \mathrm{~Hz}$ et $2.4 \mathrm{~Hz}$, respectivement.

- La réduction de la fréquences propres pour un état de précontrainte partielle par rapport à un état de précontrainte totale se montaits (influence des masses additionnelles éliminée):

\section{Poutres en béton léger (Phase 4):}

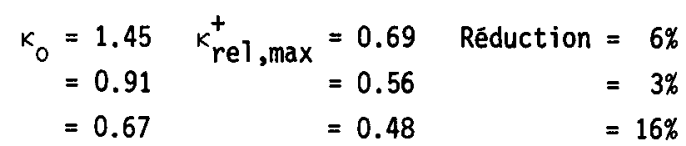

Poutres en béton normal (Phase 4):

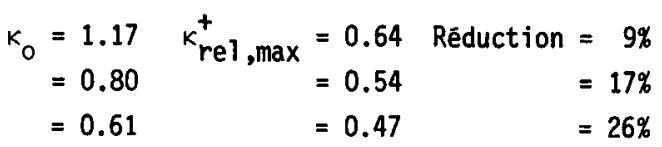

La réduction était ainsi considérablement inférieure pour le béton léger que pour le béton normale.

- L'histoire de la charge avait une grande importance sur les fréquences propres.

\section{Amortissement}

\section{Dans un état précontrainte totale:}

- L'amortissement se réduisait aux cours des essais entre le moment où la force de précontrainte était modifiée et le moment où les essais étaient effectués. L'amortissement atteignait, environ 48 heures après modification de la force de précontrainte, d'une manière asymptotique la valeur la plus basse. Par rapport à une heure après la modification de la force de précontrainte, 1 'amortissement était de $27 \%$ inférieur pour du béton léger et de $20 \%$ inférieur pour du béton normal.

La valeur minimale d'amortissement atteinte se montait (par rapport à l'amortissement critique): Pour la poutre en béton léger $\xi=c / c_{c r}=0.4 \%$, pour la poutre en béton $\xi=c / c_{c r}=0.7 \%$.

$L$ 'amortissement de la poutre en béton léger totalement précontrainte était ainsi de $44 \%$ inférieur à celui de la poutre analogue en béton normal.

- L'amortissement était pratiquement indépendant de la déflexion relative et de l'importance de la force de précontrainte centrée, cette-à-dire pratiquement indêpendante de la hauteur des sollicitations (dans le domaine quasi-élastique).

- L'amortissement était indépendant de la fréquence.

Dans un état de précontrainte partielle:

Il est à remarquer, que les valeurs d'amortissement sont à réduire d'environ de moitié pour des poutres 
non-précontraintes selon [1], si le pourcentage d'armature passive de $0.57 \%$ est à peu près triplé. La même chose serait à attendre pour des poutres partiellement précontrainte.

- D'une maniẻre analogue aux poutres non-précontrainte, l'amortissement dominua fortement pour des sollicitations relatives superieures et tendait asymptotiquement vers un minimum.

Variation du coefficient d'amortissement: pour le bẻton léger entre $\xi=2.5 \%$ et $\xi=1.0 \%$, pour le béton normal entre $\xi=4.0 \%$ et $\xi=1.5 \%$.

L'amortissement était augmenté en cas de précontrainte partielle (après la formation complète des fissures) par rapport à la précontrainte totale d'un facteur 6 dans le cas de petites sollicitations relatives et d'un facteur 2 dans le cas de grandes sollicitations relatives. L'ordre de grandeur de l'amortissement $n$ 'a été influencée que d'une façon insignificante par l'injection du cáble de précontrainte.

- L'amortissement augmenta, pour les poutres dont les cábles de précontrainte n'étaient pas injectés, d'environ $30 \%$ pour les poutres en béton léger en réduisant le degré de précontrainte de la poutre au repos de 1.45 a 0.67 , et d'environ $40 \%$ pour la poutre en béton normal en réduisant le degré de précontrainte de la poutre au repos de 1.17 à 0.61 .

- L'amortissement des poutres en béton léger partiellement précontrainte était de 30\% inférieur ả celui des poutres en béton normal pour de mêmes dégrés de précontrainte de la poutre au repos.

\section{Dans un état de non-précontrainte:}

Selon [1] il est à remarquer, que les valeurs d'amortissement pour des pourcentages d'armatures élévês peuvent être très inférieures aux valeurs données ici (comparē paragraph 1.4).

- Le coefficient d'amortissement varia: pour la poutre en béton léger entre $\xi=3.5 \%$ et $\xi=2.0 \%$, pour la poutre en bēton normal entre $\xi=4.5 \%$ et $\xi=3.5 \%$.

- La correspondance avec les poutres, de la première série d'essais de ce projet de recherche, qui avaient le même pourcentage d'armature passive (LB 1 et BI dans [1]) est bonne. 
By victue of lightweight concrete as a new building material and the recent techniques of prestressing, structures of extraordinary slenderness have become feasible, which are prone to vibrations of large amplitudes. The "Dynamic Behaviour of Lightweight and Normalweight Reinforced Concrete Beams" has hence made a project of research at the Institute of Structural Engineering of the Swiss Federal Institute of Technology (ETH) Zurich, Switzerland. As a part of this project, tests were conducted on too prestressed beams, one of lightweight concrete and the other of normalweight concrete, that supplement an older test series finished in 1979 on reinforced concrete beams without prestress.

The current tests of this report aimed at a better understanding of the effects three key parameters (degree of prestress, cracking, level of excitation) have on the dynamic properties (i.e. flexural rigidity, natural frequencies, damping characteristics) of lightweight and normalweight concrete beams when they are fully, or partially prestressed, and respond quasi-elastically.

The beams investigated were $8.4 \mathrm{~m}$ long with a cross-section of $0.4 \mathrm{~m}$ width and $0.24 \mathrm{~m}$ height. They were simply supported, could be prestressed to a variable degree, and were tested for their dynamic properties by standard vibration decay methods. Excitation was achieved by a vibrating mass in midspan with servohydraulic control. The beams's flexural rigidity could also be measured under static load providing an equivalent deflection from the at-rest position.

The research programme comprised the following four phases for each of the too beams:

- Phase 1:

The beams were tested with reduced free span of $1=5.40 \mathrm{~m}$ (and cantilevering ends of $1.68 \mathrm{~m}$ on either side) under full prestress. Six straight and unbonded prestressing rods permitted to apply a centric prestressing force of variable magnitude.

- Phase 2:

The span was increased to $7.92 \mathrm{~m}$. The prestressing rods were first tensioned to provide full prestress, then removed to permit cracking, and finally tensioned to restore full prestress, closing the cracks. This sequence gave bracketing values of the dynamic behaviour. Afterwards, some of the straight rods were replaced by a parabolic tendon to investigate the influence of cable profile.

- Phase 3:

The degree of prestress in the beams at rest could be gradually reduced by applying additional weights on their top, thus permitting tests under partial prestress, the curved tendon being still ungrouted.

- Phase 4:

Analogous to phase 3 , but after grouting of the tendon.

An overview of the test results is given below, with the notation as follows: $k$ designates the degree of prestress in midspan, $k_{0}$ in the at-rest position, $k_{r e l, m a x}^{+}$at maximum relative deflection downwards from the at-rest position. Conclusions comparing lightweight and normalweight concrete are indicated by a vertical bar at the left margin.

\section{Flexural Rigidity}

- Amount of reduction of dynamic rigidity from fult to partial prestressing:

\section{Lightweight concrete beams (Phase 4):}

$\begin{aligned} K_{0} & =1.45 & K_{\text {rel, } \max }^{+} & =0.69 & \text { reduction } & =11 \% \\ & =0.91 & & =0.56 & & =15 \% \\ & =0.67 & & =0.48 & & =28 \%\end{aligned}$

\section{Normalweight concrete beams (Phase 4):}

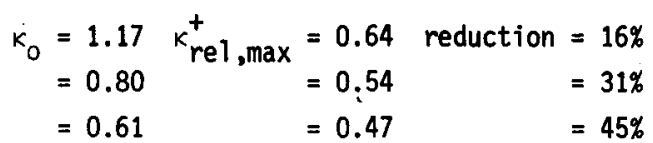

The decay of flexural rigidity was considerably less for lightweight concrete than for normalweight concrete.

- The static flexural rigidity (secant modulus) was $5 \%$ to $10 \%$ less for both kinds of concrete beams than the dymamic one in the investigated frequency range $(f=2$ to $6 \mathrm{~Hz}$ ). This difference increased to $10 \%$ at small deflections from at-rest. 
- Both the dynamic and the static rigidity of the lightweight concrete beam was $32 \%$ (full prestress) resp. $28 \%$ (no prestress) smaller than those of the normalweight concrete beam.

- The loading history had a large effect on the rigidity (both static and dynamic).

\section{Natural Frequencies}

- The natural frequencies of the lightweight concrete beam ranged from $9.8 \mathrm{~Hz}$ (full prestress, $1=5.04 \mathrm{~m}$ span) to $2.4 \mathrm{~Hz}$ (partial prestress, additional weights, $1=7.72 \mathrm{~m}$ span), those of the normalweight concrete from $11.25 \mathrm{~Hz}$ to $2.4 \mathrm{~Hz}$.

- Reduction of frequency from full to partial prestress (compensated for additional weights):

Lightweight concrete beams (Phase 4):

$\begin{aligned} \kappa_{0} & =1.45 & k_{\text {rel, } \max }^{+} & =0.69 & \text { reduction } & =6 \% \\ & =0.91 & & =0.56 & & =8 \% \\ & =0.67 & & =0.48 & & =16 \%\end{aligned}$

\section{Norma Iweight concrete beams (Phase 4):}

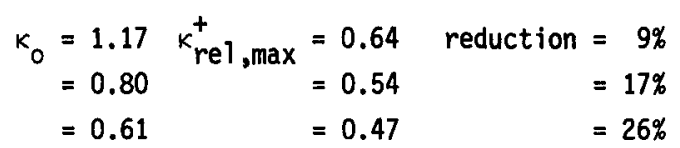

The reduction of frequency was considerably less for lightweight concrete than for normalweight concrete.

- The loading history had a large effect on the frequency as well.

Damping

In the state of full prestress:

- The damping decreased with time passed between instant of change in prestress and test. About 47 hours after change in prestress, the damping had asymptotically approached its minimum, being $27 \%$ lower (1ightweight concrete) resp. $20 \%$ lower (normalweight concrete) than one hour after changing the magnitude of prestress. Minimum of the damping coefficient (with respect to critical): lightweight concrete $\xi=c / c c r$ $=0.4 \%$, norma Iweight concrete $\xi=c / c_{c r}=0.7 \%$.

The damping for full prestress was $44 \%$ lower for lightweight concrete than for normalweight concrete.

- The amount of damping changed negligibly with relative deflection or with the magnitude of the prestressing force. That is, it was virtually independent of the stress level (within the quasi-elastic range).

- The damping was independent of frequency.

\section{In the state of partial prestress:}

Remembering that for non-prestressed beams the damping was roughly halved by trippling the percentage of reinforcement (from initially $\rho=0.57 \%$, of [1]), about the same could be expected for partially prestressed beams.

- Similar to the observations on non-prestressed beams, the damping decreased rapidly with higher levels of relative loading and tended asymptotically to a minimum. Range of the damping coefficient: lightweight concrete beams $\xi=2.5$ to $\xi=1.0 \%$, normalweight concrete beams $\xi=4 \%$ to $\xi=1.5 \%$.

Compared to full prestress, partial prestress (after previous complete cracking) enhanced the damping by a factor of 6 for small relative loading and a factor of 2 for large relative loading. Grouting the prestressing tendon did not significantly change the order of magnitude of damping.

- Reducing the degree of prestress while the tendon was ungrouted increased the damping: for the beam of lightweight concrete by about $30 \%$ when $\kappa_{0}$ dropped from 1.45 to 0.67 (with reference to at-rest), for that of normalweight concrete by about $40 \%$ when $k_{0}$ dropped from 1.17 to 0.61 .

- For the same degree of prestress at-rest, the damping in the 1ightweight concrete beam was about $30 \%$ smaller than in the normalweight concrete beam. 


\section{In the state of no prestress:}

From previous tests [1] it is known that damping may be much less for higher percentages of reinforcement than the current results suggest (see Section 1.4).

- Range of the damping coefficient:

lightweight concrete

$$
\zeta=c / c \text { cr: }=3.5 \text { to } 2.0 \%
$$

normalweight concrete

- For the same percentage of reinforcement (non-prestressed), these results compare well with previous data (beams LB1 and B1) from the first series of tests [1] of this research project. 
Der vorl iegende Versuchsbericht ist im Rahmen des Forschungsprojektes "Dynamisches Verhalten von Leichtbeton- und Betonkonstruktionen" als Tei1 2 ausgearbeitet worden. Dieses Projekt wurde durch einen finanziel len Beitrag der Firma

Hunziker \& Cie AG, Baustoffabriken, Zürich,

ermöglicht. Für die grosszügige Unterstützung möchten die Verfasser der Firma Hunziker und insbesondere den Herren Direktoren W. Thut, Dipl. Ing. ETH, und E. Setz, Dipl. Ing. ETH, herzlich danken.

Für die Veberlassung der benötigten Bewehrungsstähle danken die Verfasser der Firma

von Moos Stahi AG, Luzern.

Bei der Versuchsdurchführung hat Herr $K$. Bucher mitgearbeitet. Herr M. Baumann, dipl. Bauing. ETH, hat die messtechnischen Belange betreut. Herr G. Göseli zeichnete die Bilder und betreute die Drucklegung. Frl. S. Burki schrieb die Druckbogen. Für die wertvolle Unterstiutzung und die gute Zusanmenarbeit danken die Verfasser allen Genannten sowie allen anderen, die zum Entstehen dieses Versuchsberichtes beigetragen haben, bestens. 
[1] Dieterle R., Bachmann H.: "Versuche über den Einfluss der Rissbildung auf die dynamischen Eigenschaften von Leichtbeton- und Betonkonbalken", Institut für Baustatik und Konstruktion, ETH Zürich, Versuchsbericht Nr. 7501-1, Dezember 1979. Birkhäuser Verlag Basel und Stuttgart.

[2] Dieterle R.: "Modelle für das Dämpfungsverhalten von schwingenden Stahlbetonträgern im ungerissenen und gerissenen Zustand", Institut für Baustatik und Konstruktion, ETH Zürich, Bericht Nr. 111, April 1981, Birkhäuser Verlag Basel und Stuttgart.

[3] Swamy R.N.: "Damping Mechanics of Cementition Systems", Chapter 30 in 'Dynamic Waves in Civil Engineering', Wiley Interscience, 1974.

[4] Rigon C., Thürlimann B.: "Fatigue Tests on Post-Tensioned Concrete Beams", Institut für Baustatik und Konstruktion, ETH Zürich, Versuchsbericht Nr. 8101-1, August 1985 (in Englisch), Birkhäuser Verlag Basel und Stuttgart.

[5] Bachmann H.: "Von der vollen zur teilweisen Vorspannung", Institut für Baustatik und Konstruktion, ETH Zürich, Bericht Nr. 132, August 1982, Birkhäuser Verlag Basel und Stuttgart.

[6] Bachmann H.: "Design of Partially Prestressed Concrete Structures Based on Swiss Experiences", Journal of the Prestressed Concrete Institute (PCI), No. 4, July/August 1984. 


\section{Bezeichnungen}

(In Klammern sind die Bezeichnungen aufgefüht, die in [1] verwendet worden sind.)

\section{Geometrische Grössen}

$\begin{array}{lll}A_{c} & \left(F_{b}\right) & \text { Leichtbeton- bzw. Betonquerschnittsfläche } \\ A_{s} & \left(F_{e}\right) & \text { Fläche der unteren schlaffen Balkenlängsbewehrung } \\ A_{s}^{\prime} & \left(F_{e}^{\prime}\right) & \text { Fläche der oberen schlaffen Balkenlängsbewehrung } \\ A_{p l} & & \text { Fläche der geraden Spannstäbe } \\ A_{p z} & & \text { Fläche der Spanndrähte im gekrümmten Spannglied } \\ A_{i} & \left(F_{i d}\right) & \text { ideelle Querschnittsfläche } \\ b & (B) & \text { Balkenbreite } \\ h & (H) & \text { Balkenhöhe } \\ I & (I) & \text { Trägheitsmoment } \\ I_{i} & \left(I_{i d}\right) & \text { Trägheitsmoment der ideellen Querschnittsfläche } \\ 1 & (1) & \text { Balkenspannweite } \\ s & & \text { Abstand der statischen Einzel kräfte vom Auflager } \\ t & & \text { Bügelabstand, Zeit } \\ x & (x) & \text { Abstand der neutralen Achse vom Druckrand } \\ y & & \text { Abstand der Schwerachse vom Oruckrand } \\ D & (D) & \text { Durchmesser }\end{array}$

\section{Kraftgrössen}

D

$F(t) \quad\left(P_{G r}\right) \quad$ Anregungskraft vor Ausschwingversuch

$F_{\text {stat }}\left(P_{s t}\right) \quad$ Einzelkraft beim statischen Belastungsversuch

M (M) Biegemoment

$M_{0} \cdot\left(M_{0}\right) \quad$ Biegemoment in Balkenruhelage infolge Balkeneigengewicht, Zusatzmassen, Schwinger und Ankerplatten

$M_{\text {rel }}\left(M_{\text {rel }}\right)$

$M_{p}$

$M_{\text {op }}$

$M_{\text {Dec }}$

$M_{r} \quad\left(M_{R}\right)$

Mro

$M_{\mathrm{pl}} \quad\left(M_{\mathrm{pl}}\right)$

$N_{p}$

P

$\mathrm{Z}_{\mathrm{c}}$

$\mathrm{Z}_{\mathrm{s}}$
Biegemoment infolge Relativdurchbiegung

Biegemoment infolge Vorspannung

Biegemoment in Balkenruhelage infolge Balkeneigengewicht, Zusatzmassen, Schwinger, Ankerplatte und Vorspannung $\left(M_{o p}=M_{0}+M_{p}\right)$

Dekompressionsmoment

Rissmoment

Rissöffnungsmoment

plastisches Moment

Normalkraft infolge Vorspannung

Vorspannkraft

Zugkraft im Beton

Zugkraft im schlaffen Stahl 


\section{Massen und Gewichte}

\begin{tabular}{|c|c|c|}
\hline$G_{s}$ & (g) & $\begin{array}{l}\text { Eigengewicht des Balkens ohne Zusatzmassen pro m' } \\
\text { Eigengewicht des servohydraulischen Schwingers }\end{array}$ \\
\hline$G_{A}$ & & Eigengewicht der Ankerplatten \\
\hline m & (m) & Masse des Balkens inkl. Zusatzmassen pro ' ${ }^{\prime}$ \\
\hline M & (G) & Masse des servohydraulischen Schwingers \\
\hline$\Delta \mathrm{M}$ & $(\Delta M)$ & Zusatzmassen pro Paket \\
\hline$a$ & & Gewicht der Zusatzmassen pro $\mathrm{m}^{\prime}$ \\
\hline$\gamma$ & & Raumgewicht von Leichtbeton und Beton \\
\hline
\end{tabular}

\section{Festigkeitswerte, Spannungen}

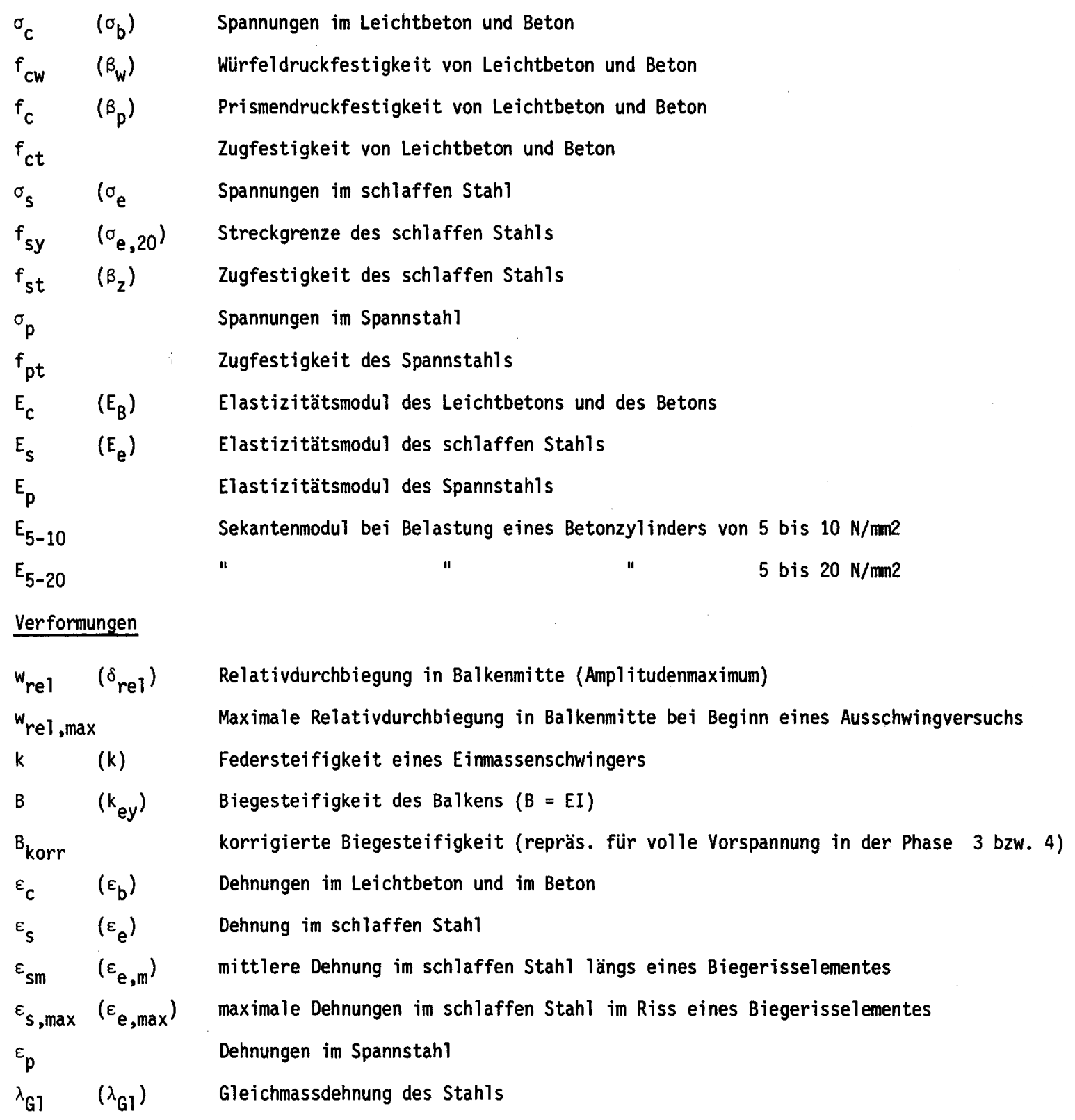




\section{Dynamische Grössen}

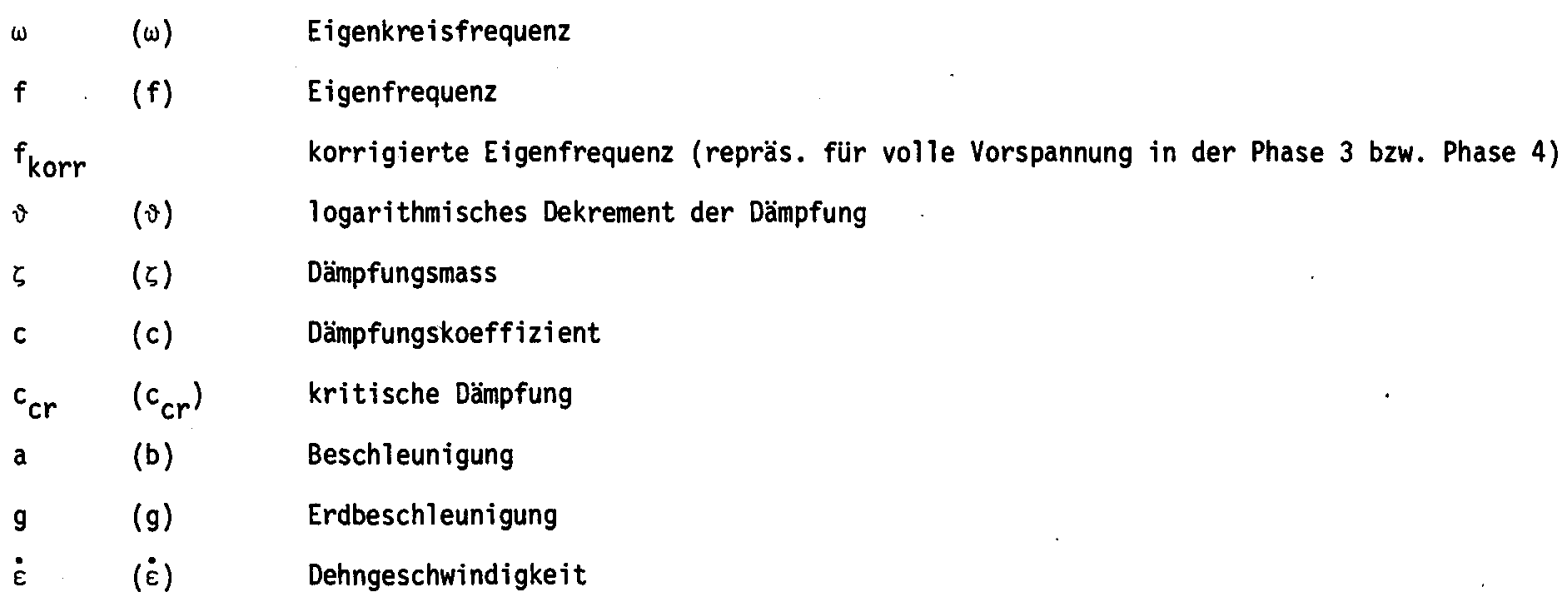

Dimensionslose Parameter

\begin{tabular}{|c|c|c|}
\hline$\rho$ & $(\mu)$ & Bewehrungsgehalt der unteren Längsbewehrung \\
\hline$\rho^{\prime}$ & $\left(\mu^{\prime}\right)$ & Bewehrungsgehalt der oberen Längsbewehrung \\
\hline$k_{0}$ & & Vorspanngrad in Balkenruhelage \\
\hline$\kappa_{\text {re }}$ & & Vorspanngrad bei maximalen Relativdurchbiegungen \\
\hline & $(\kappa)$ & Verbundkoeffizient \\
\hline
\end{tabular}

\section{Hochgeste 11 te Indizes}

Durchbiegungen nach unten

Durchbiegungen nach oben 


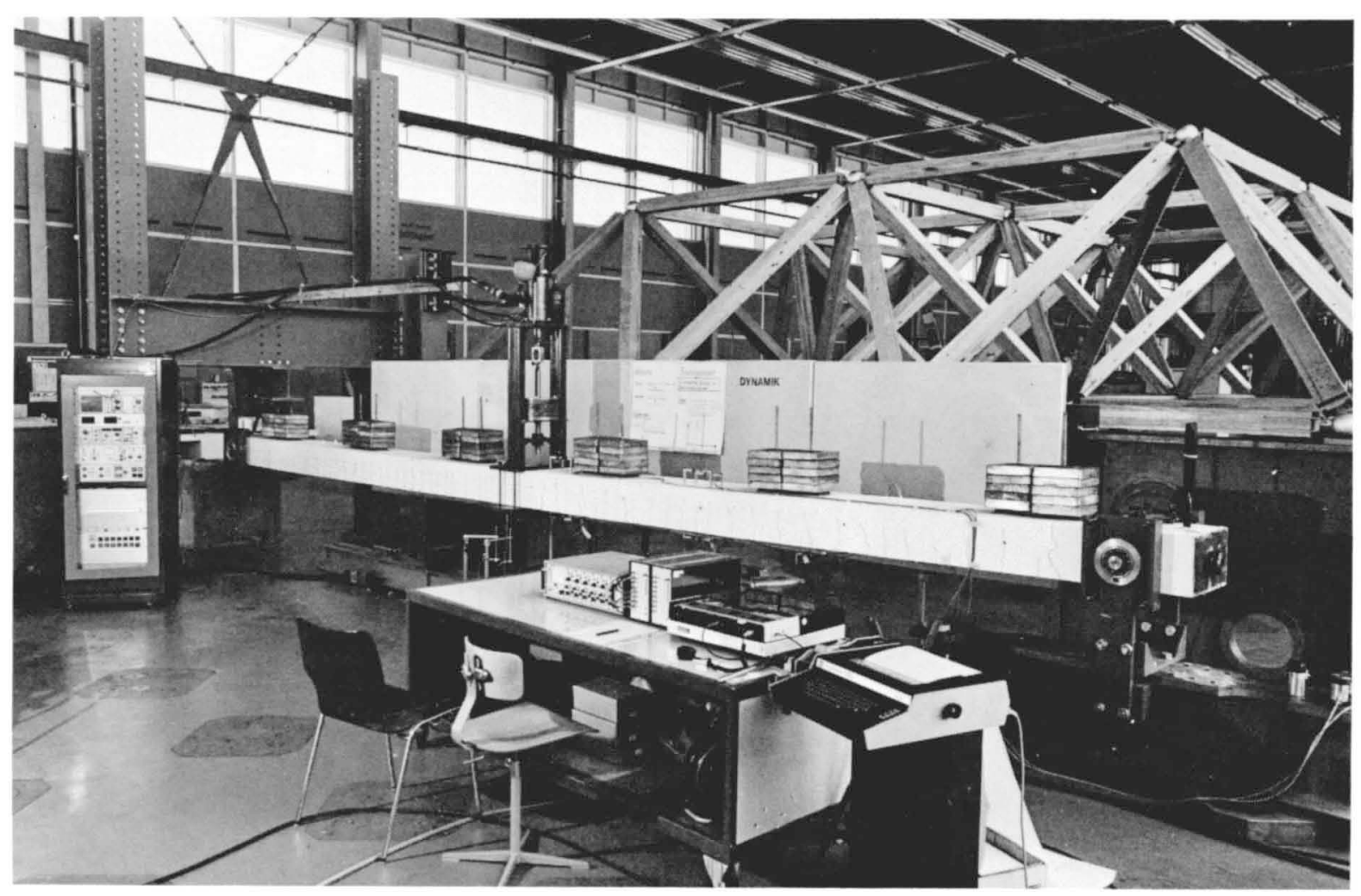

Bild 1: Versuchsanlage (Teilphase 3.3) 


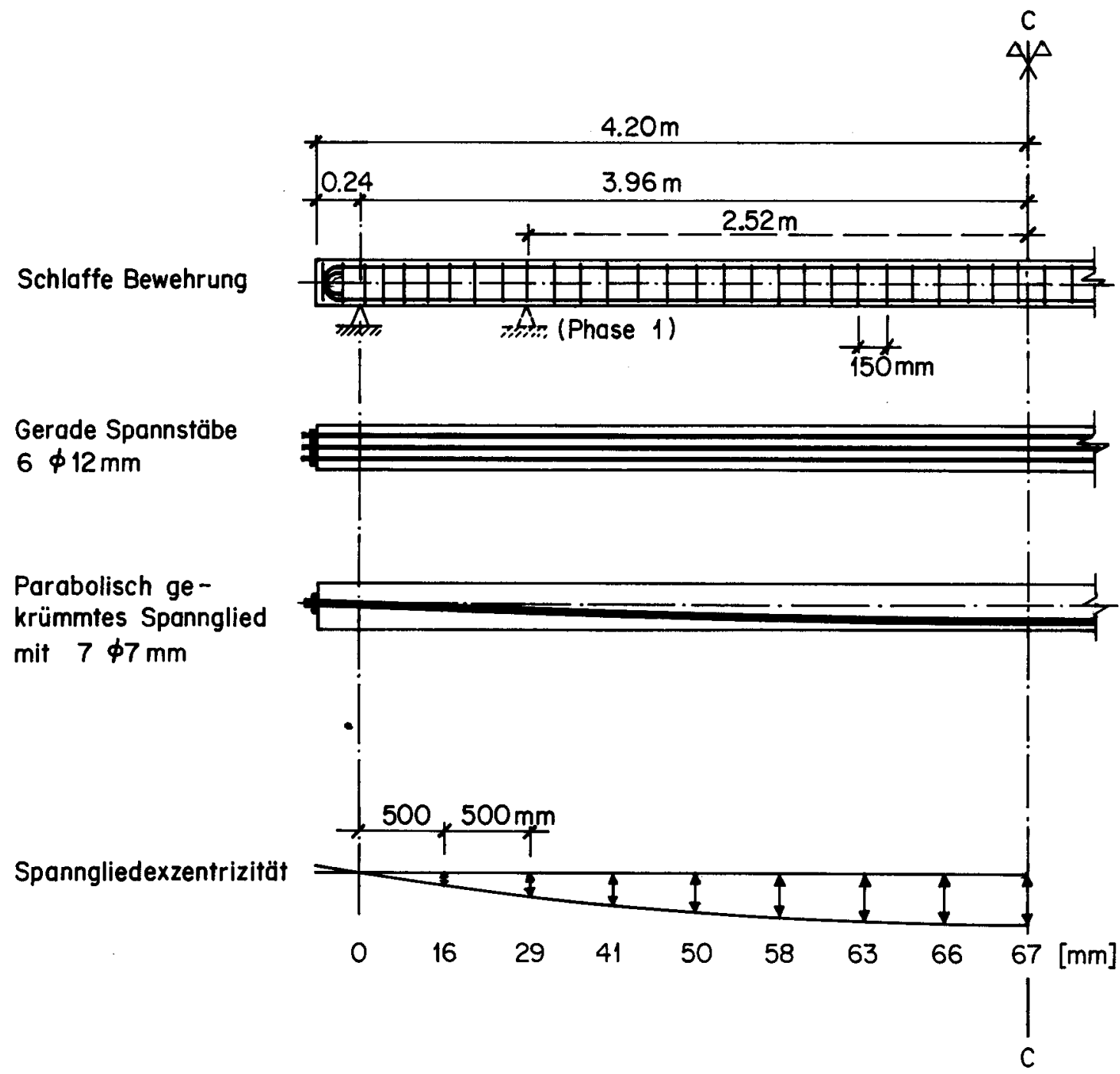

Schnitt C-C :

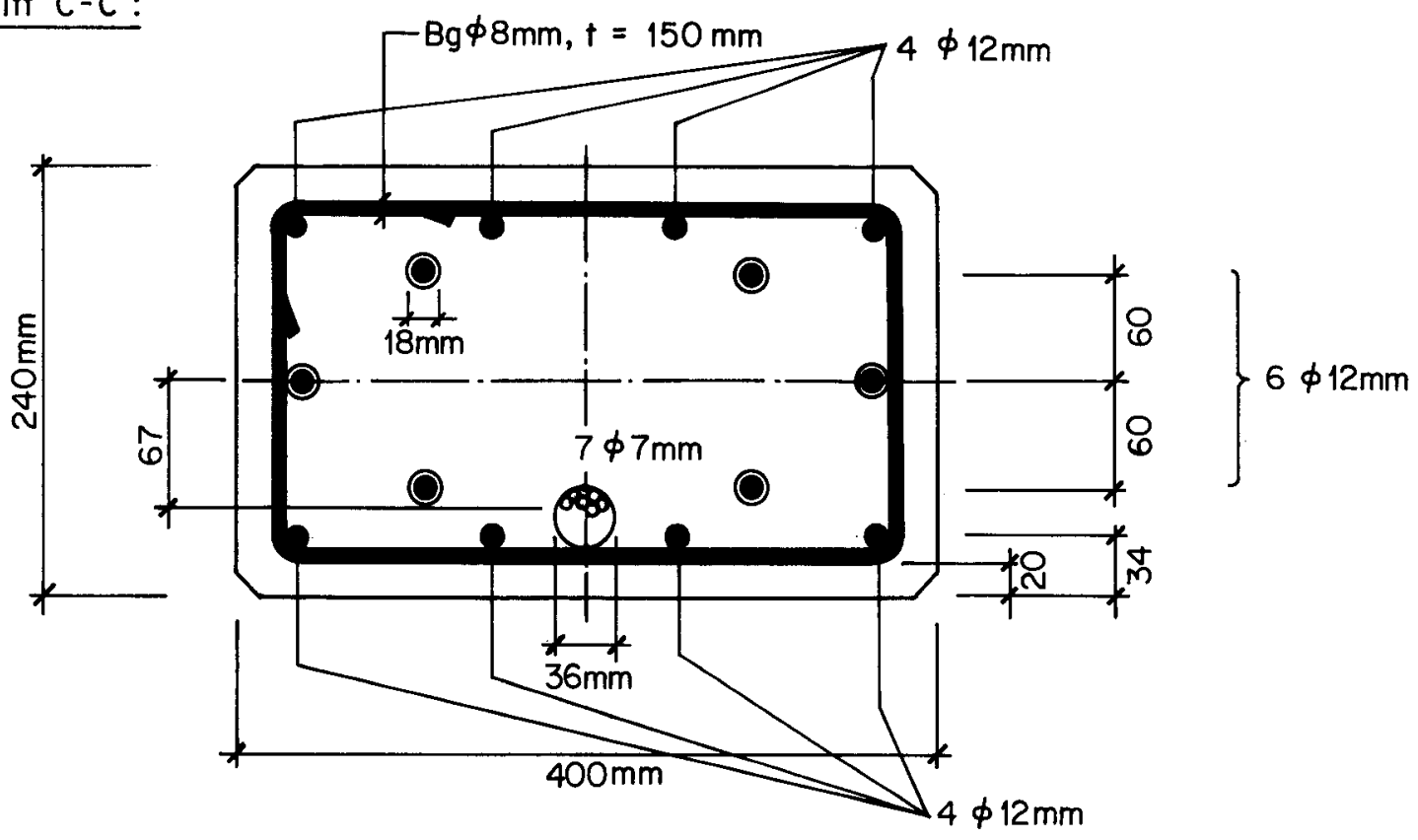

Bild 2 : Abmessungen und Bewehrungen 

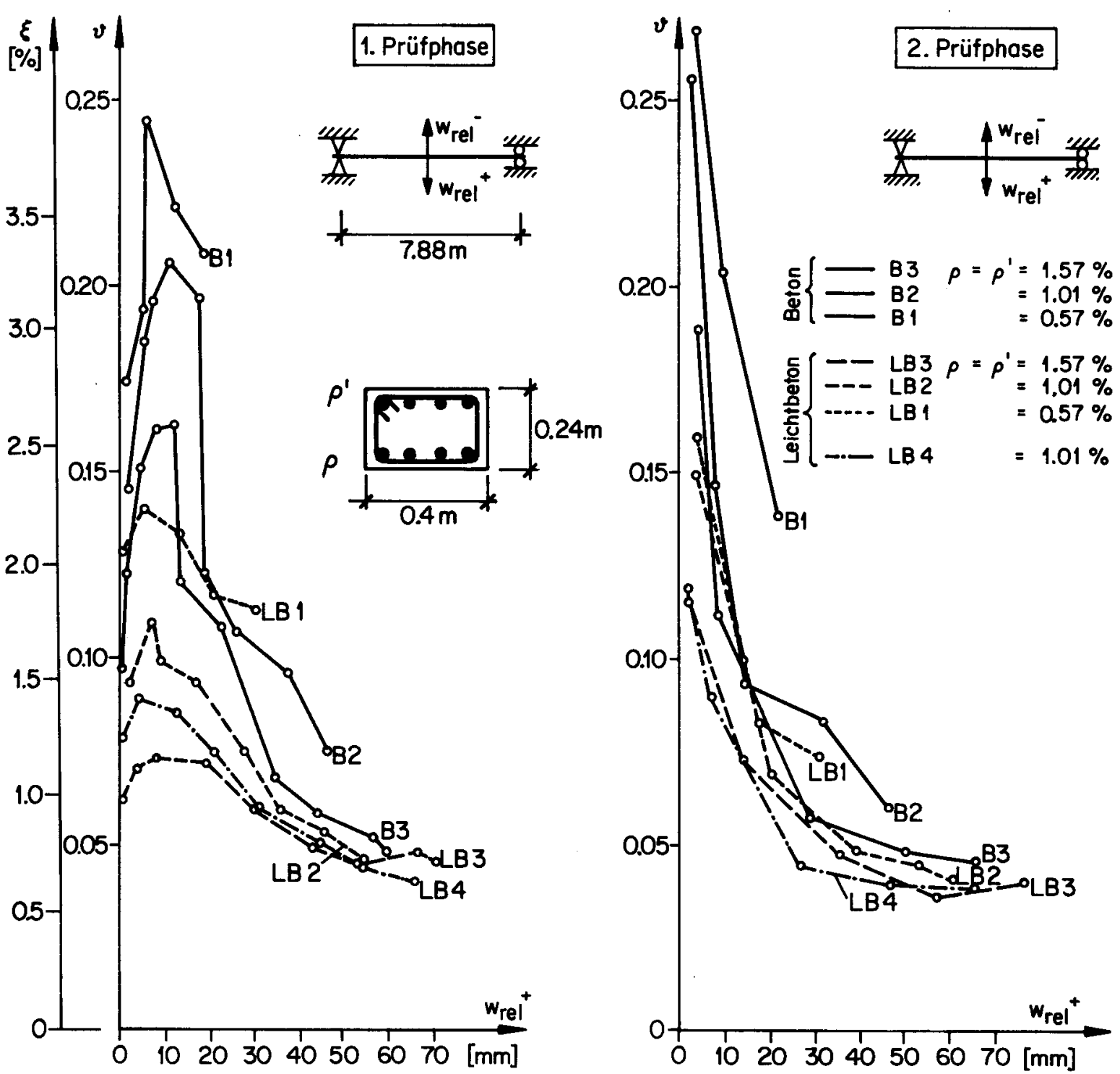

Bild 3: Dämpfung (logarithmisches Dekrement $v$ bzw. Dämpfungsmass $\xi$ ) schlaff bewehrter Balken in Funktion der Relativdurchbiegung $w_{\text {rel }}{ }^{+}$ in Balkenmitte (aus [1]) 
Biegeelement

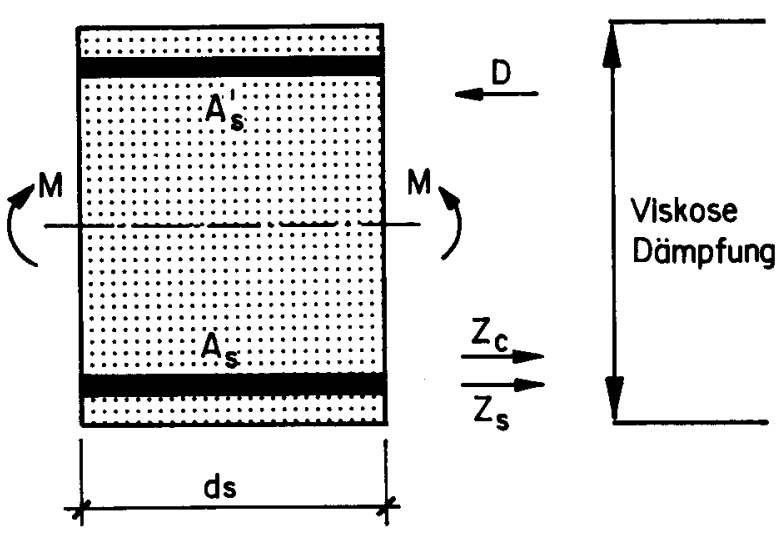

Gerissenes Biegeelement

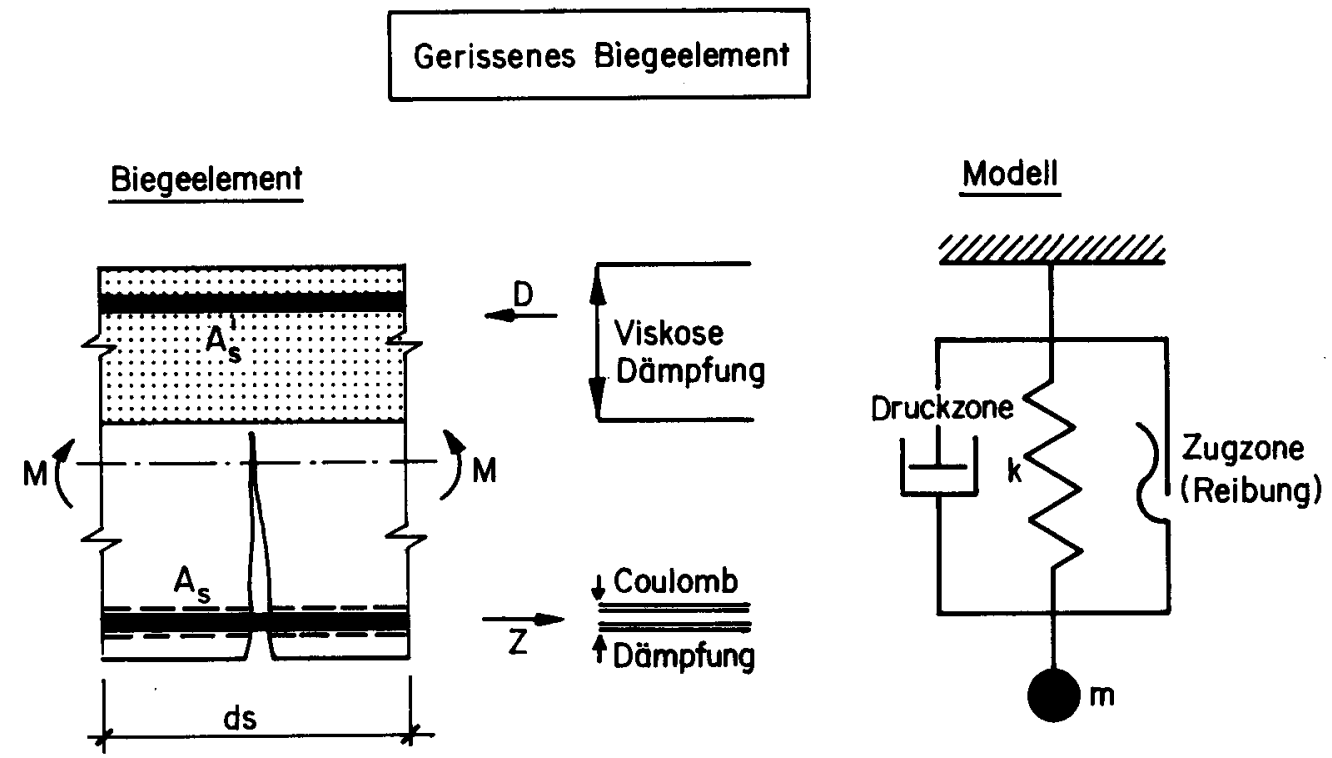

Bild 4 : Dämpfungsmodelle für das ungerissene und das gerissene Biegeelement (aus [2]) 


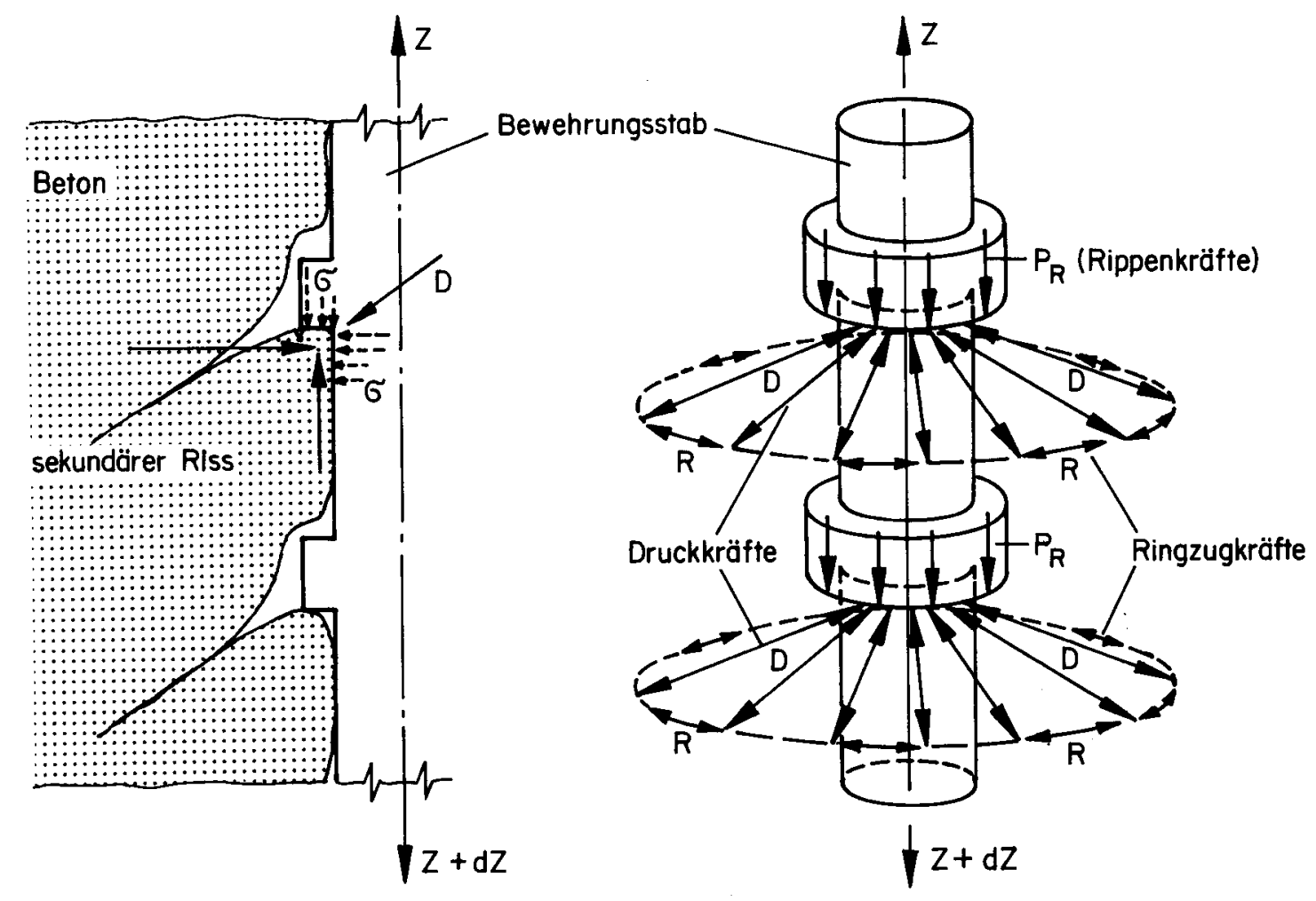

Bild 5 : Übertragung der Verbundspannungen (aus [2])

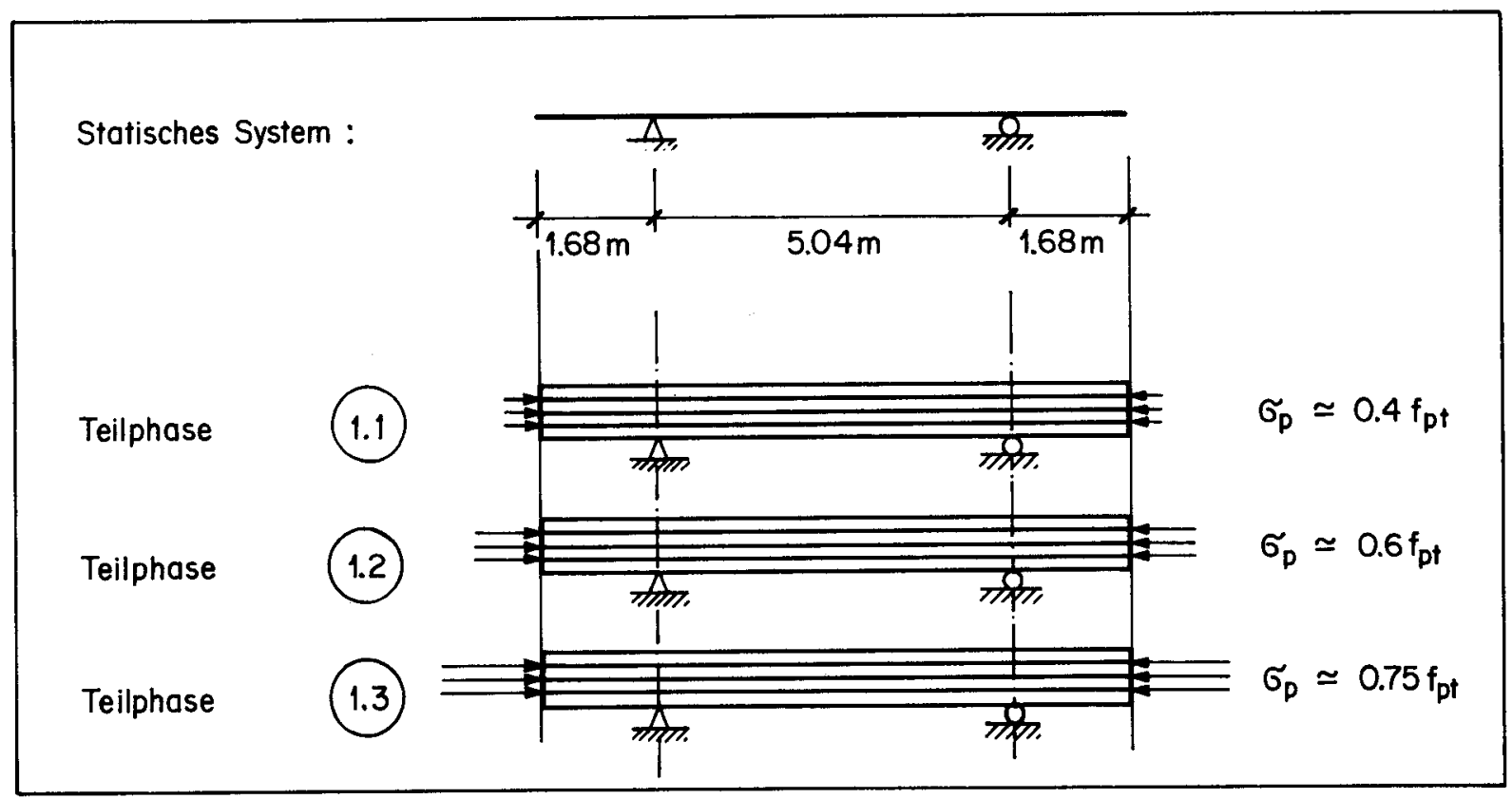

Bild 6a: Übersicht Phase 1 (schematisch) 


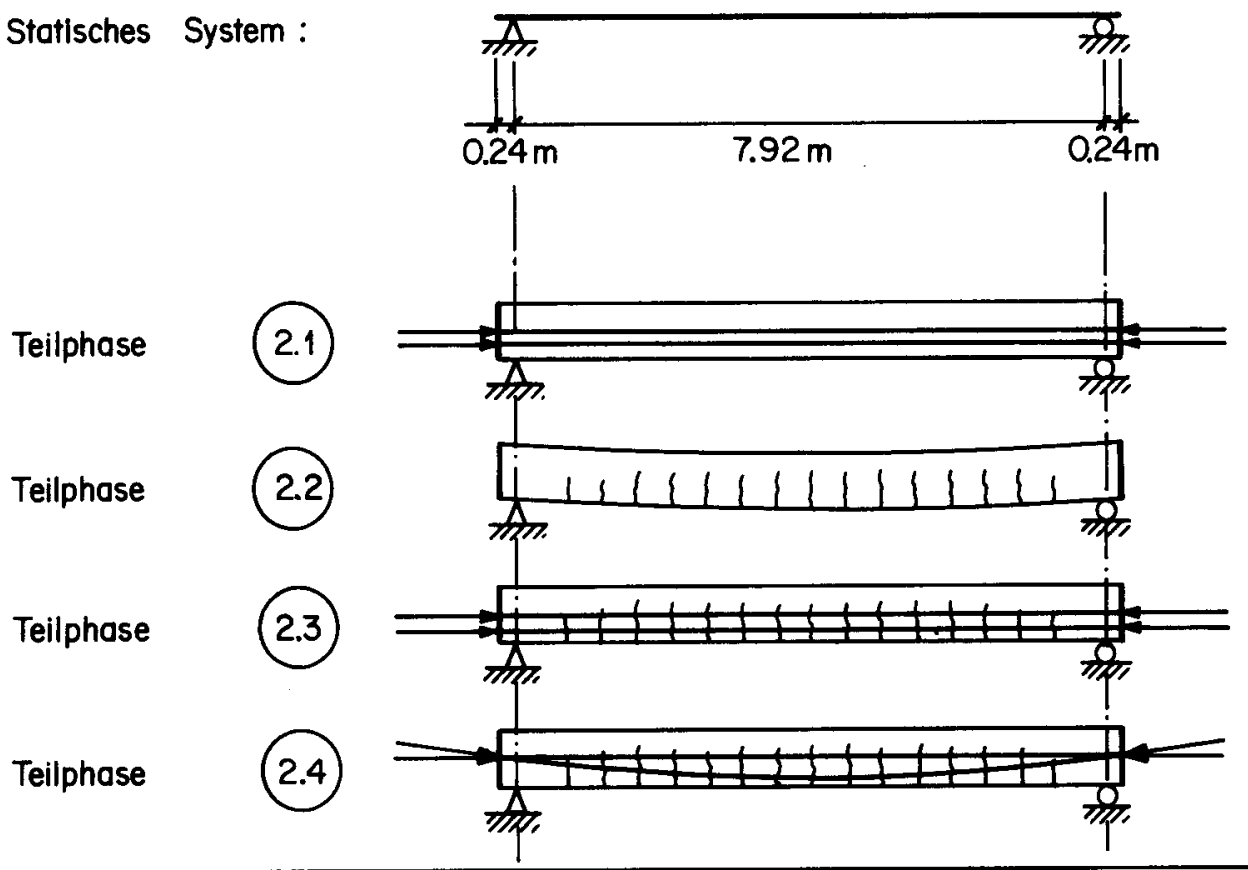

Bild 6b : Übersicht Phase 2 (schematisch)

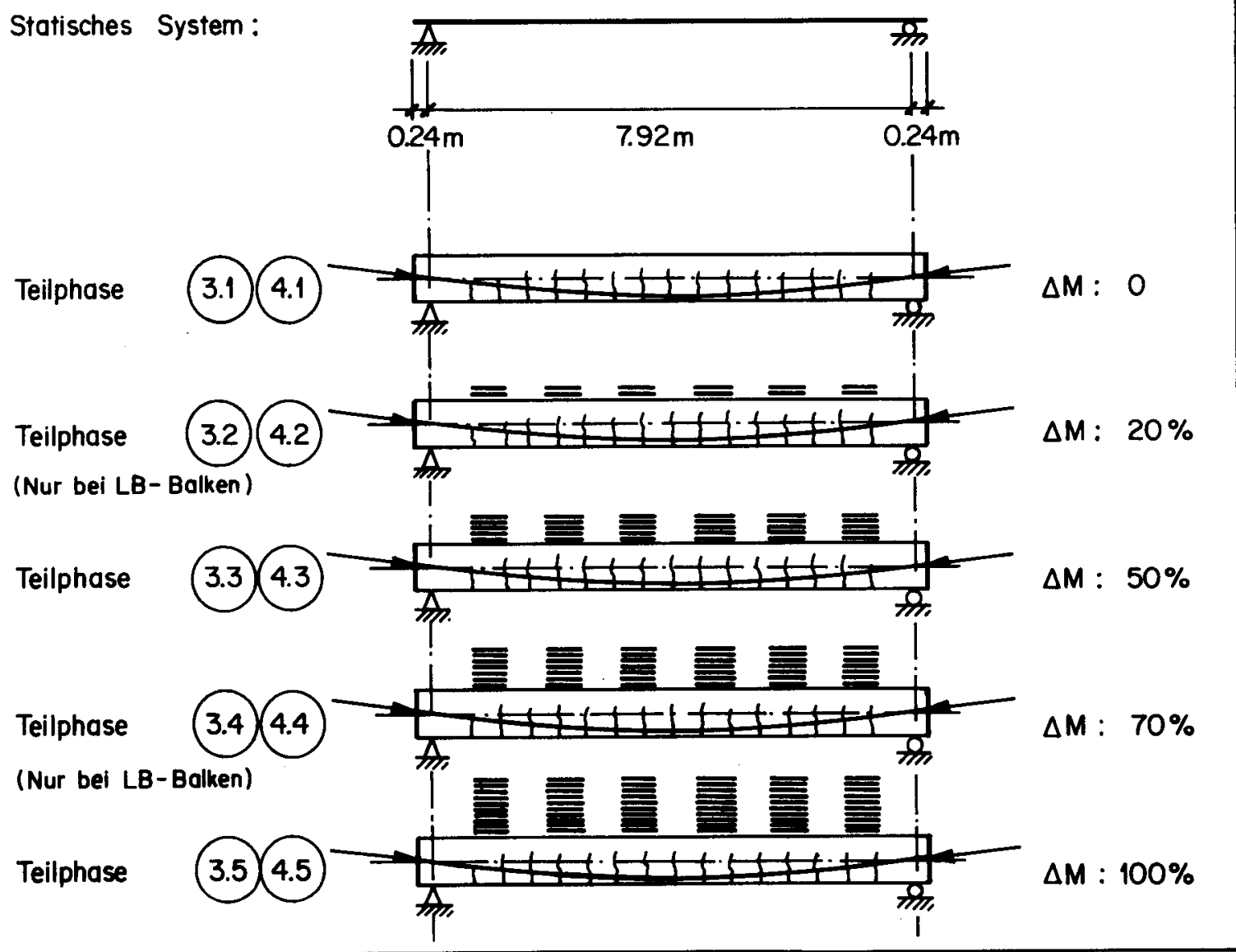

Bild 6c : Übersicht Phasen 3 und 4 (schematisch) 

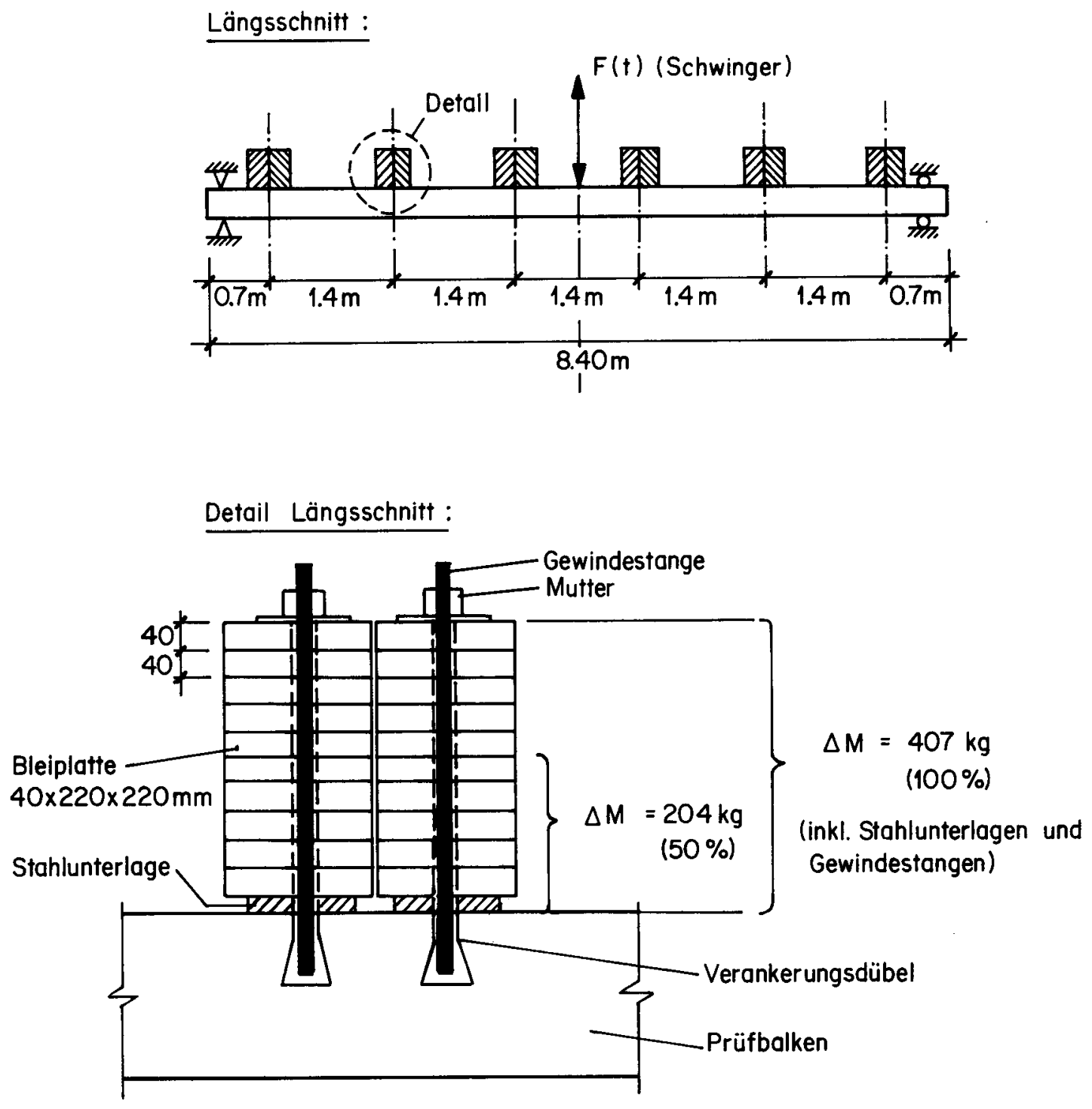

Detail Grundriss :

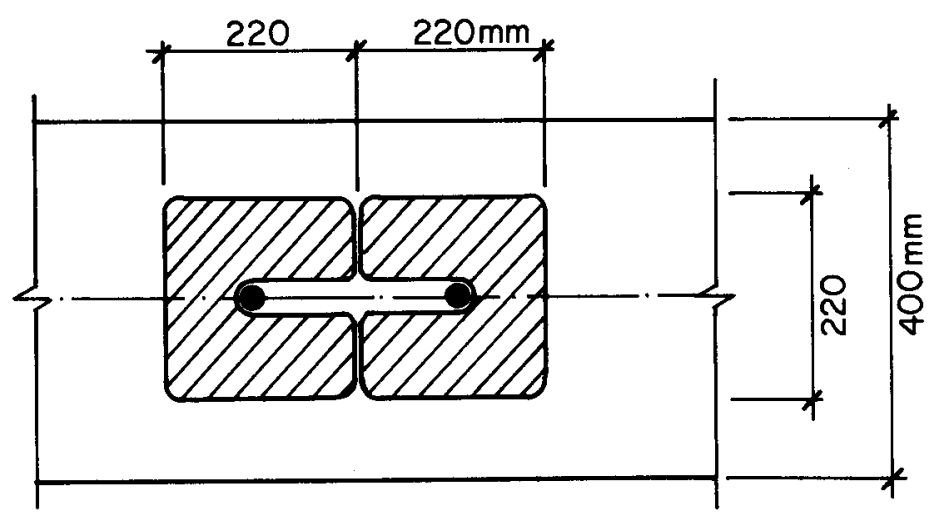

Bild 7: Lage und Anordnung der Zusatzmassen 

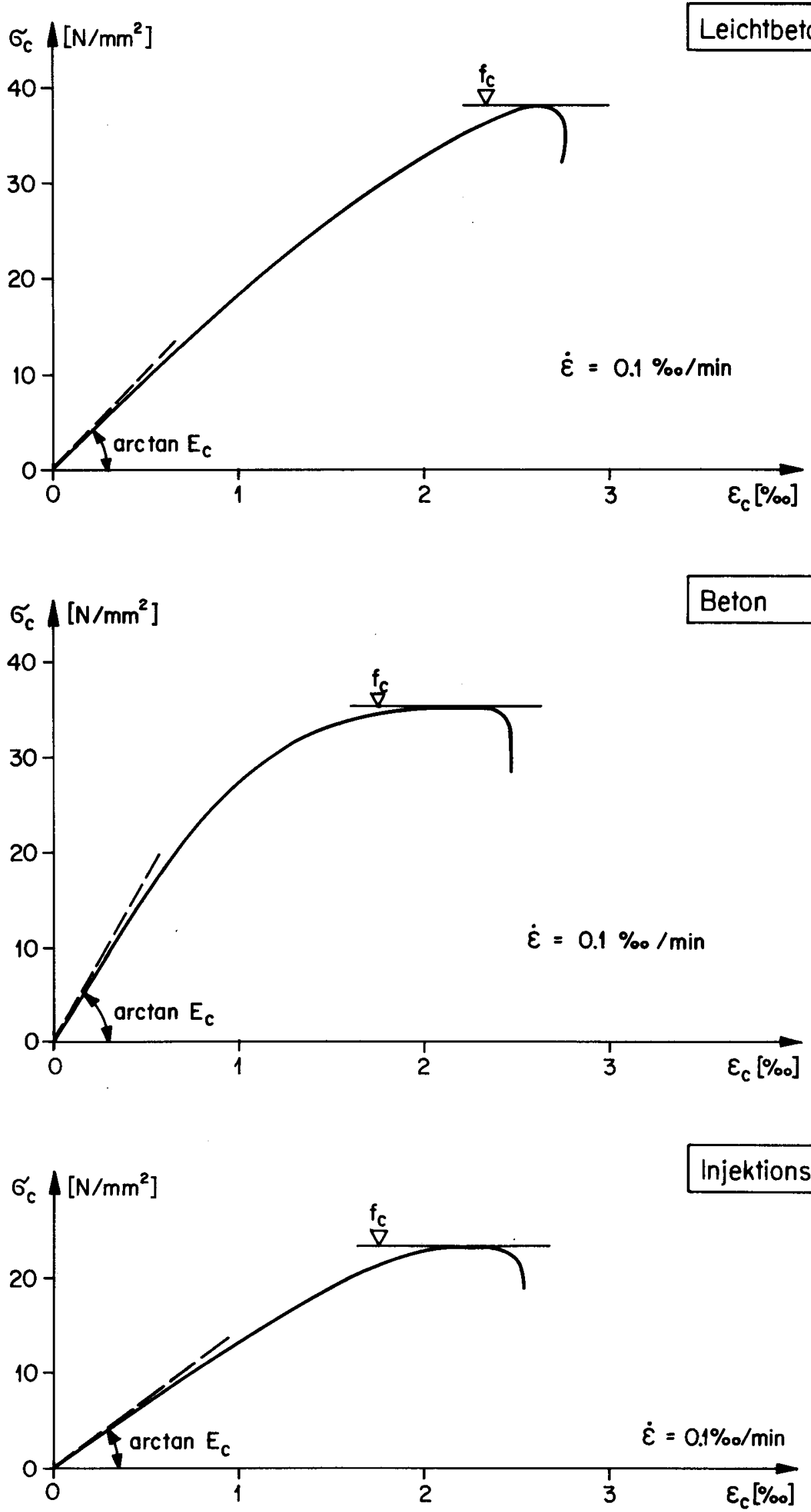

Bild 8: Spannungs-Dehnungs-Diagramme der Betone 


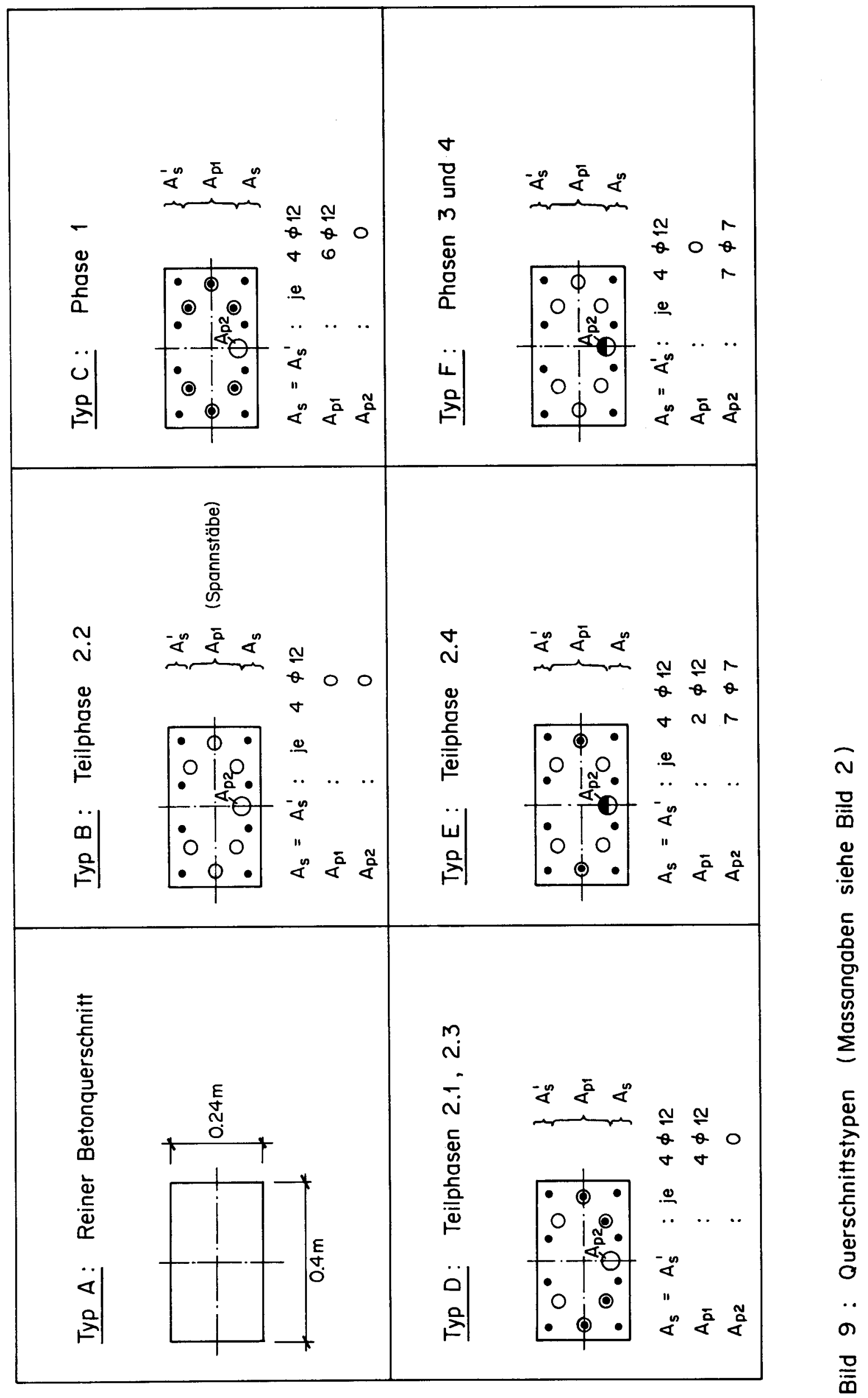




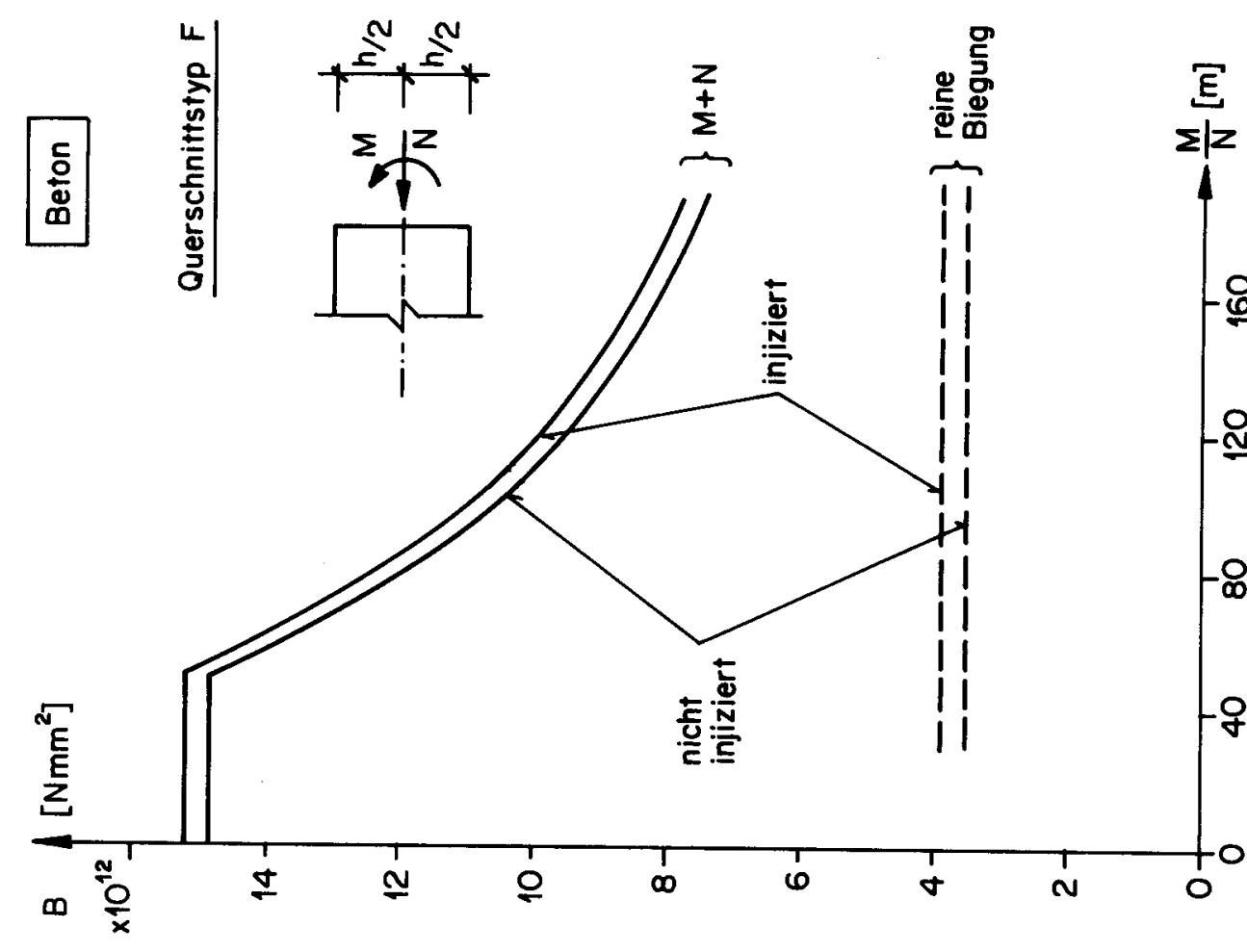

क

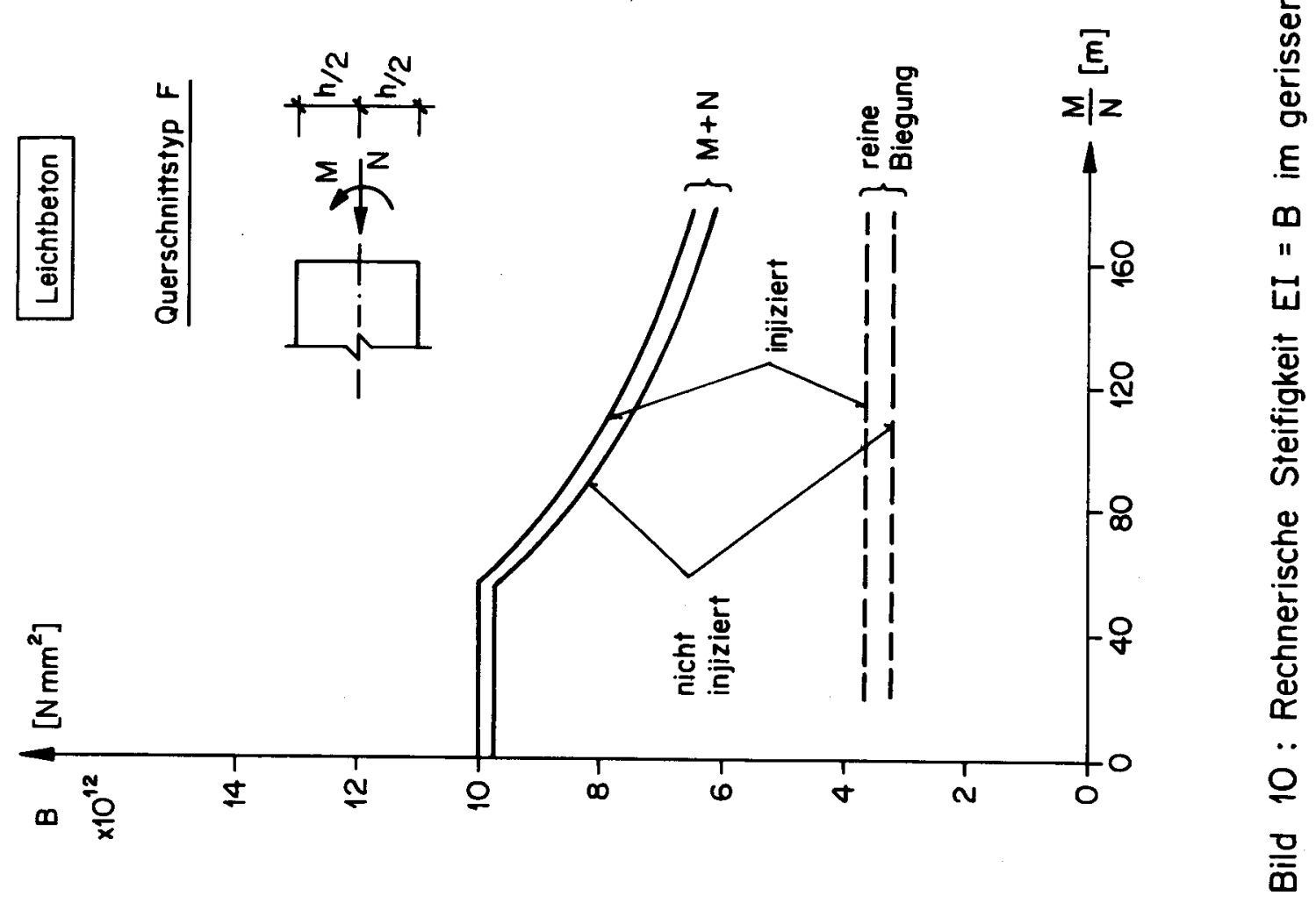




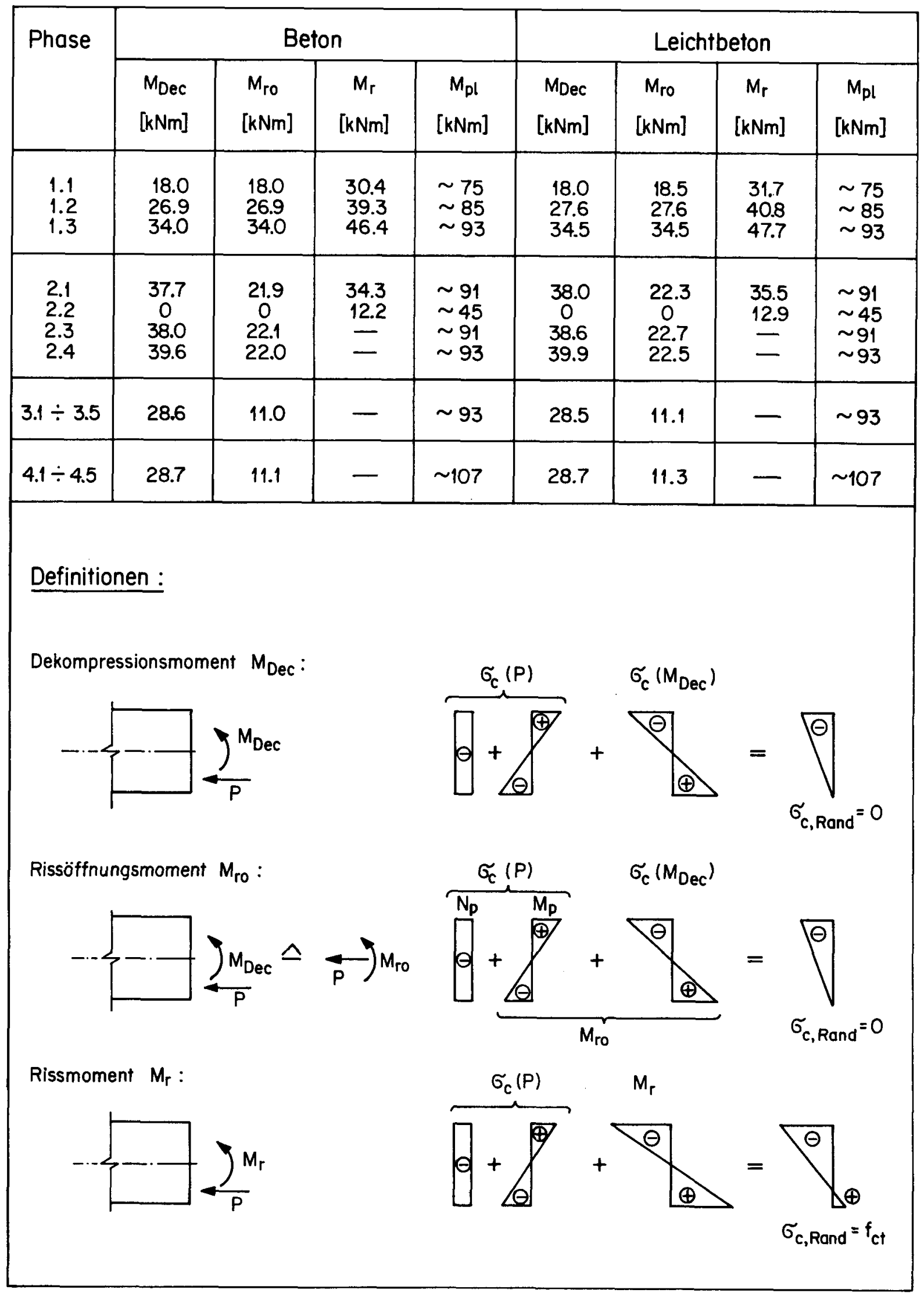

Bild 11 : Dekompressionsmomente $M_{\text {Dec }}$, Rissöffnungsmomente $M_{\text {ro }}$, Rissmomente $M_{r}$ und plastische Momente $M_{p l}$, Rechnerische Werte und Definitionen 


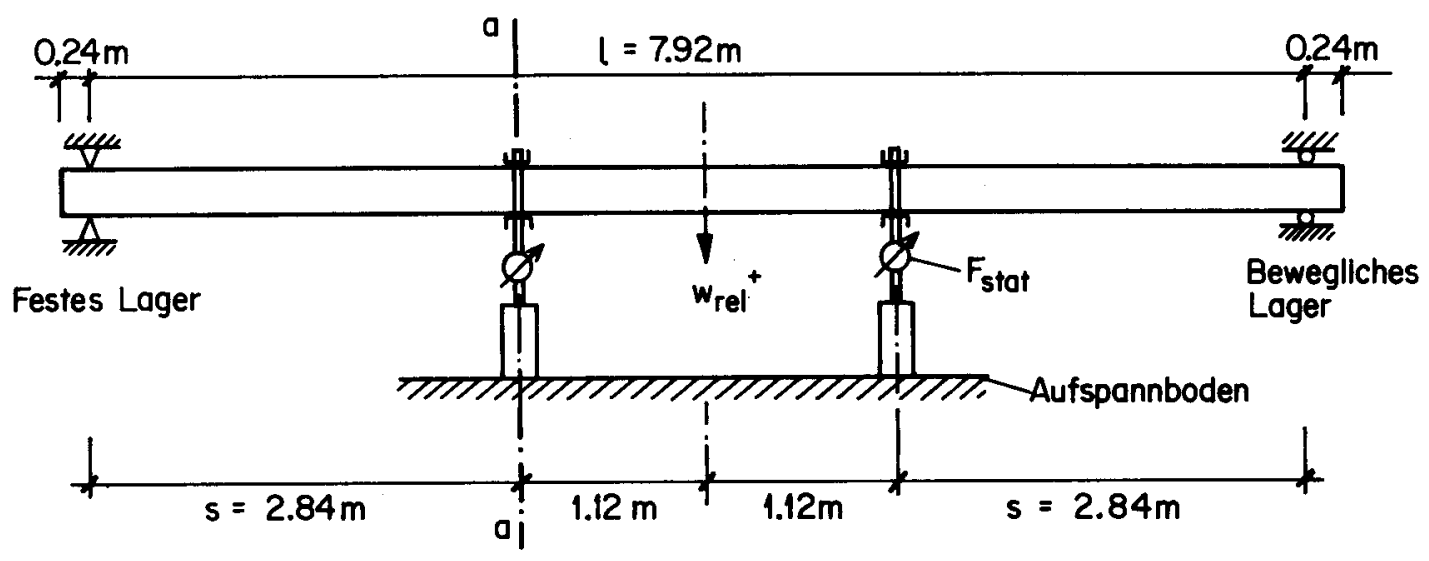

Schnitt $a-a$ schematisch :

Durchbiegungen nach oben

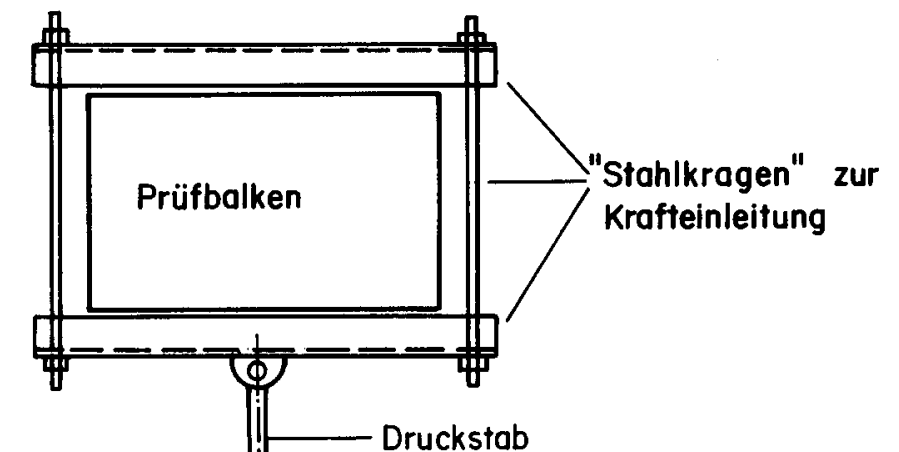

Durchbiegungen nach unten

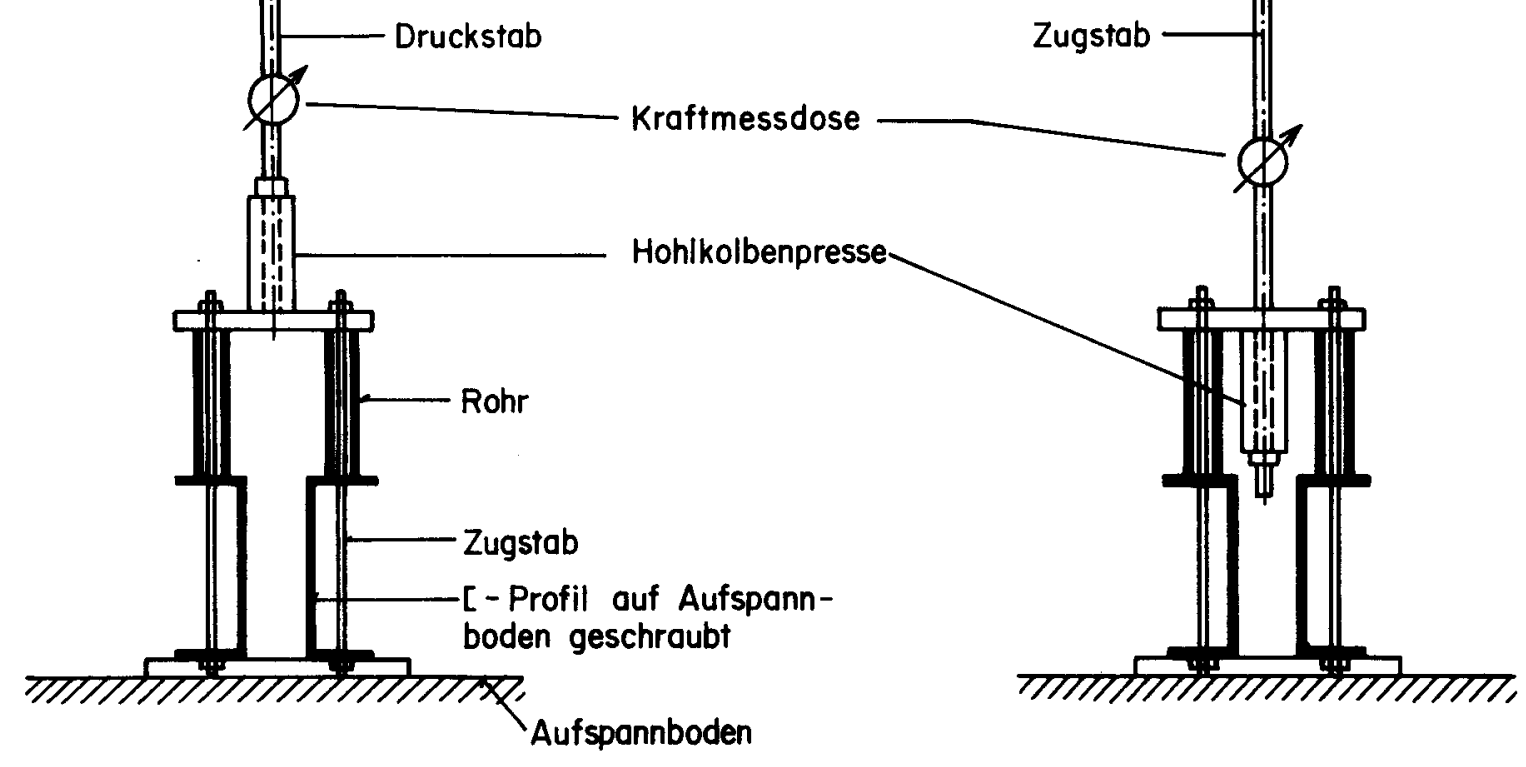

Bild 12 : Statische Belastungseinrichtung (schematisch) 


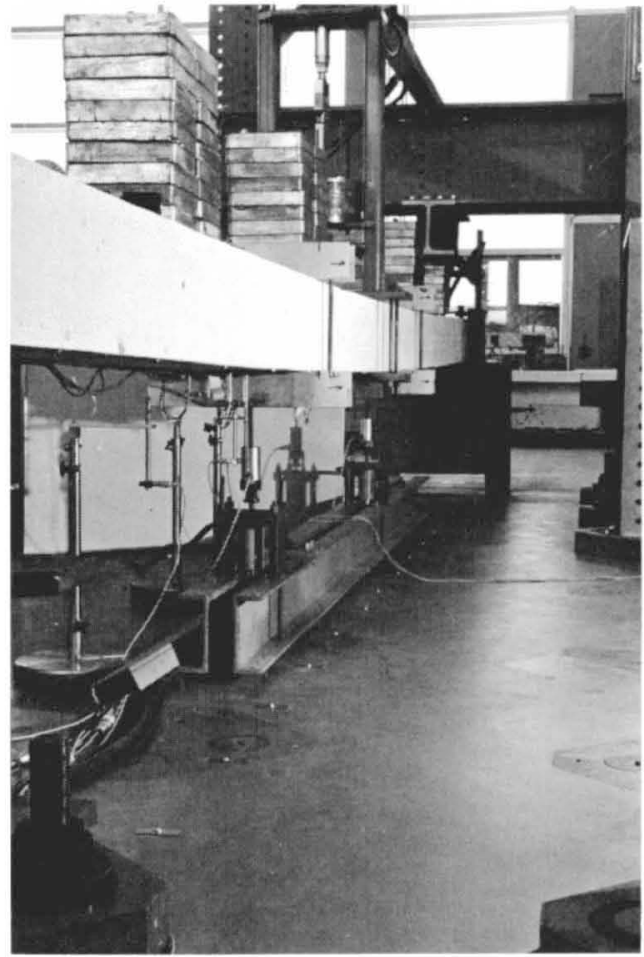

Bild 13: Statische Belastungseinrichtung (Abbildung für Relativ durchbiegungen nach unten)

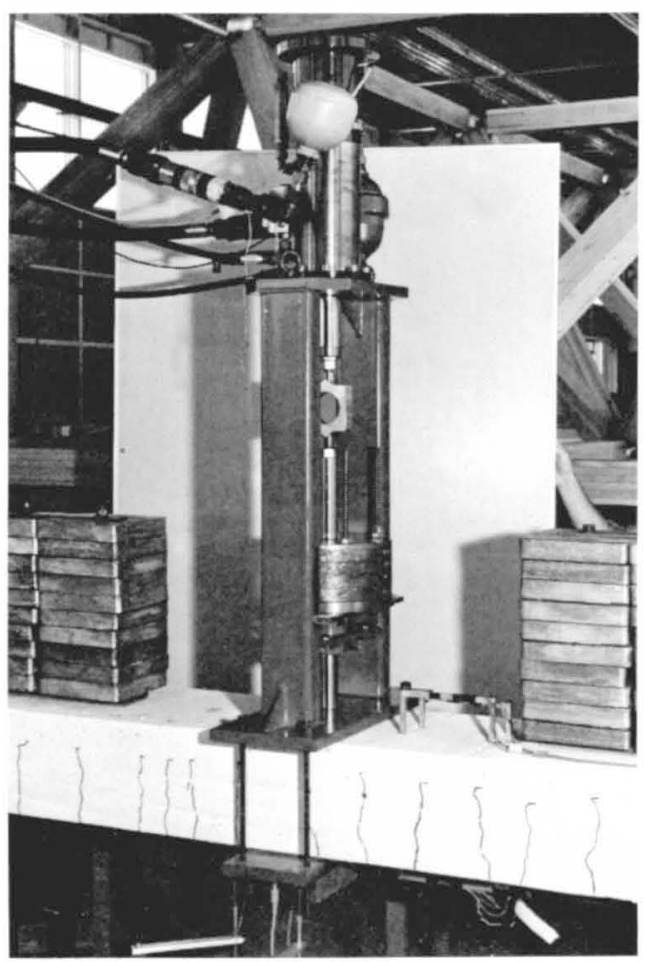

Bild 15: Servohydraulischer Schwinger

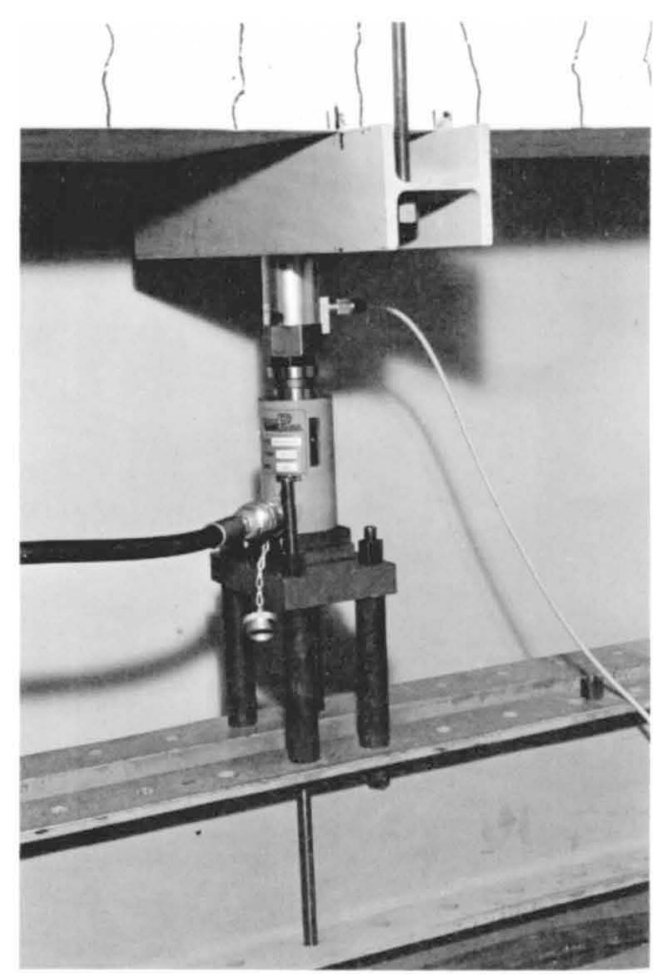

Bild 14 : Detail der stat. Belastungsein richtung (Abbildung für Relativdurchbiegungen nach oben)

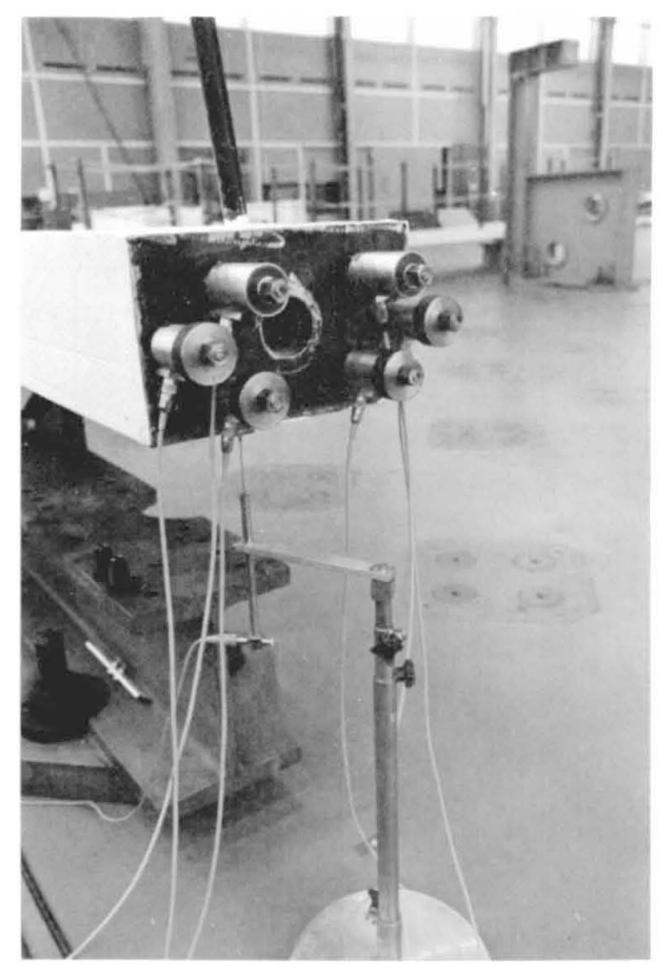

Bild 16: Ankerplatte Kraftmessdosen für Spannkraftmessung in den Spannstäben (Phase 1) 


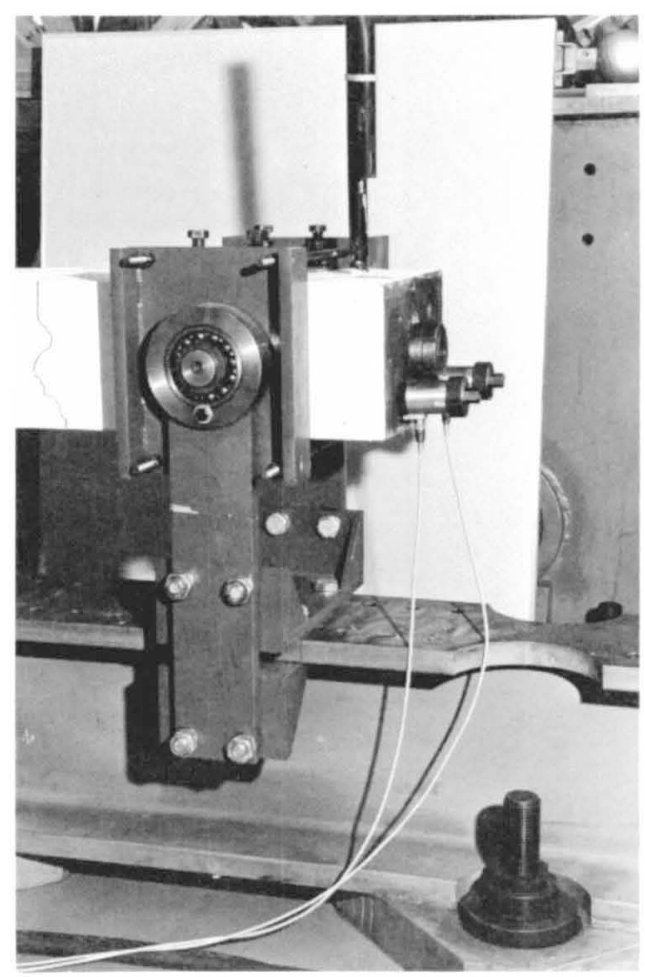

Bild 17: Festes Lager

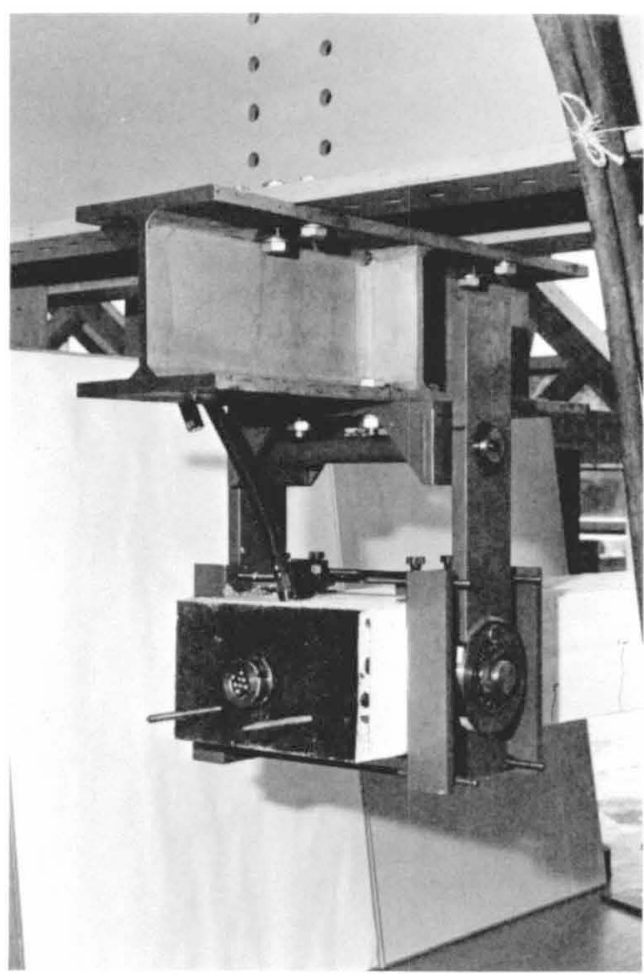

Bild 18: Bewegliches Lager

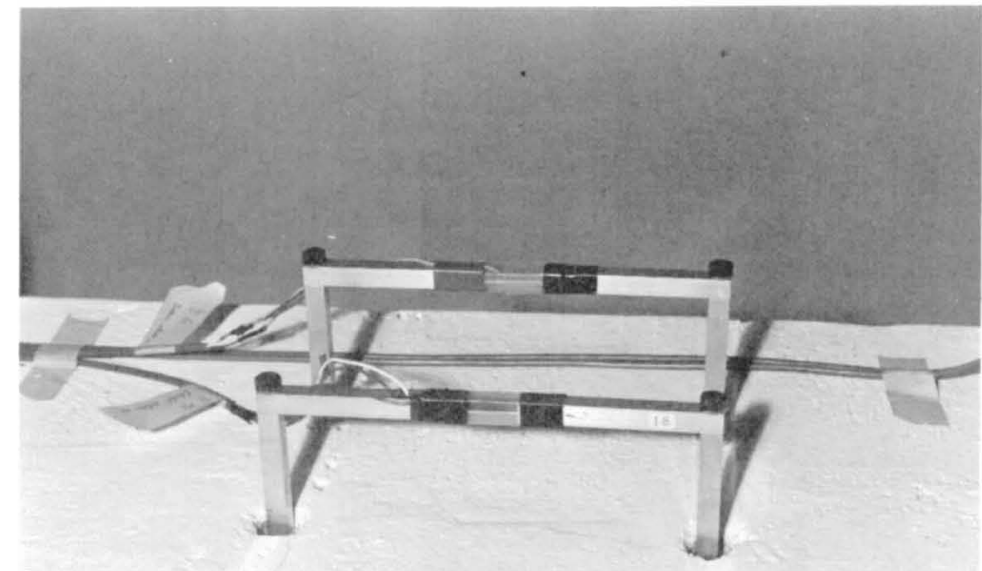

Bild 19: Dehnmessrahmen für Dehnungsmessung von Beton und Stahl 

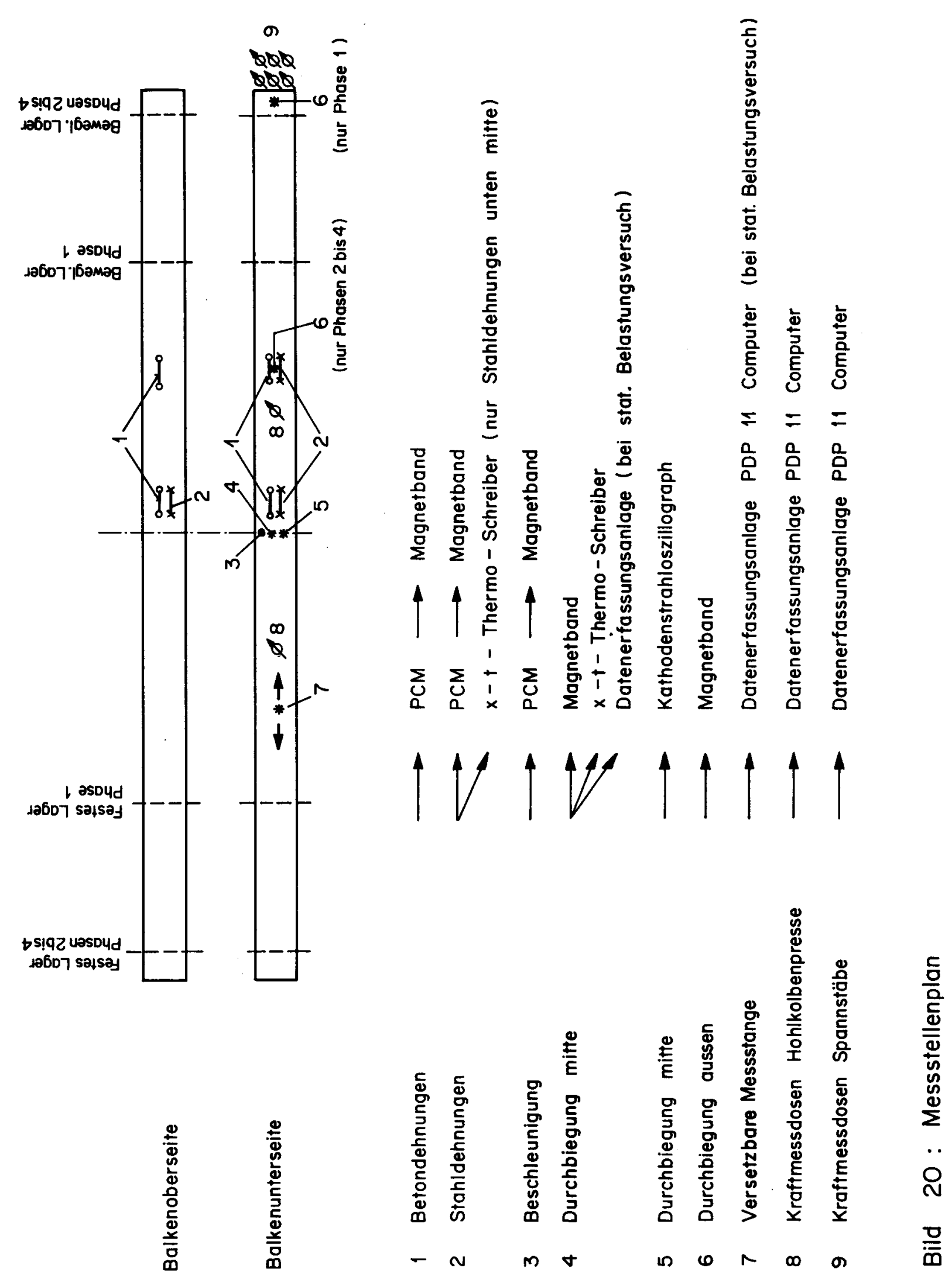


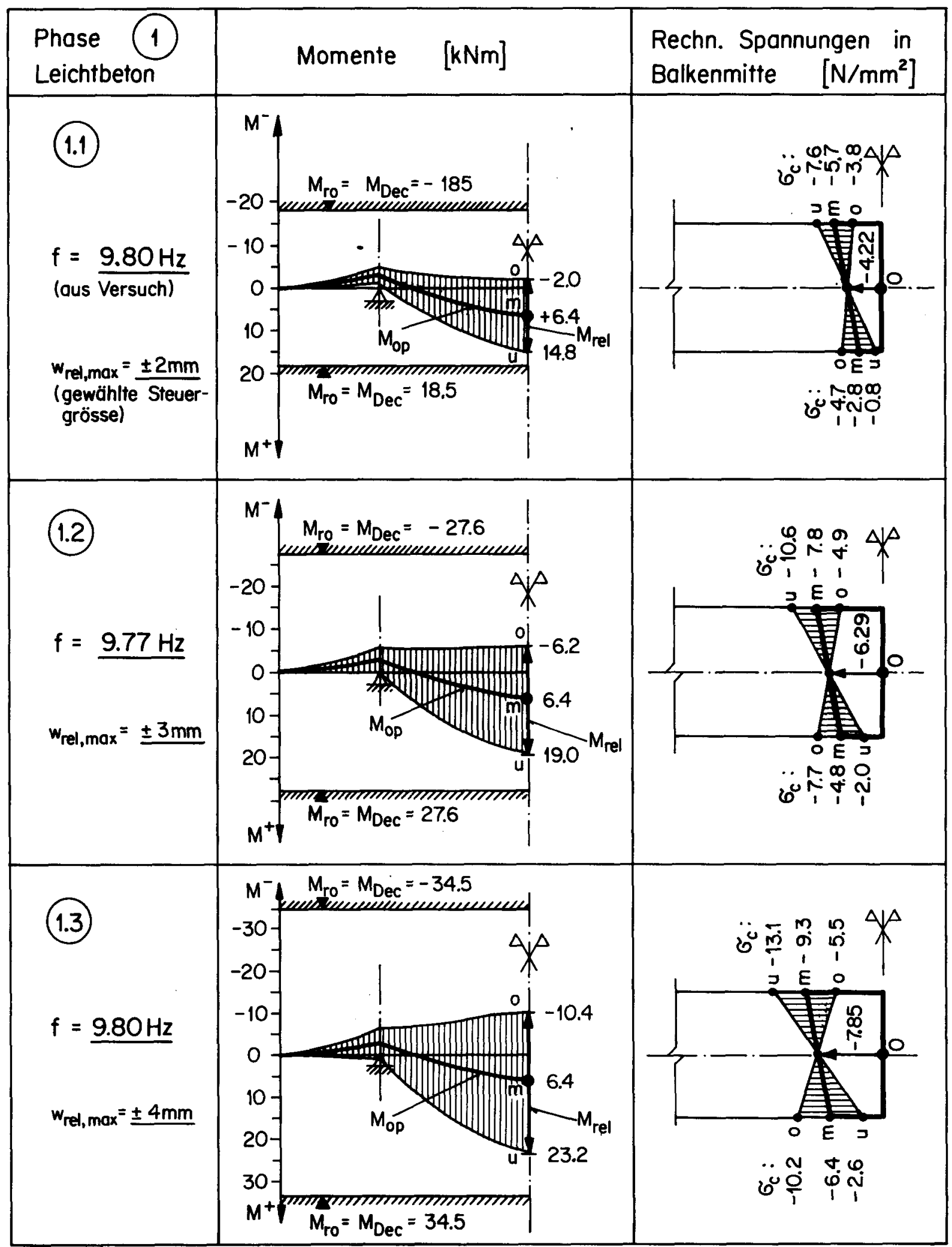

Annahmen: Biegesteifigkeit unabhängig von Relativdurchbiegung und konstant längs Balkenachse

Bild 21a : Verlauf der Biegebeanspruchungen in Balkenruhelage $M_{o p}$ (inf. Balkeneigengewicht, Zusatzmassen, Schwinger und Vorspannung) sowie bei maximaler Relativdurchbiegung $M_{\text {rel }}$, Phase 1, Leichtbetonbalken 


\begin{tabular}{|c|c|c|}
\hline $\begin{array}{l}\text { Phase (1) } \\
\text { Beton }\end{array}$ & Momente $\quad[\mathrm{kNm}]$ & $\begin{array}{l}\text { Rechn. Spannungen in } \\
\text { Balkenmitte } \quad\left[\mathrm{N} / \mathrm{mm}^{2}\right]\end{array}$ \\
\hline $\begin{array}{l}\text { (1.1) } \\
f=11.4 \mathrm{~Hz} \\
\text { (aus Versuch) } \\
w_{\text {rel,max }}= \pm 1 \mathrm{~mm} \\
\begin{array}{c}\text { (gewählte } \\
\text { grösse) }\end{array}\end{array}$ & $M_{\mathrm{rO}}=\mathrm{M}_{\mathrm{Dec}}=-18.0$ & 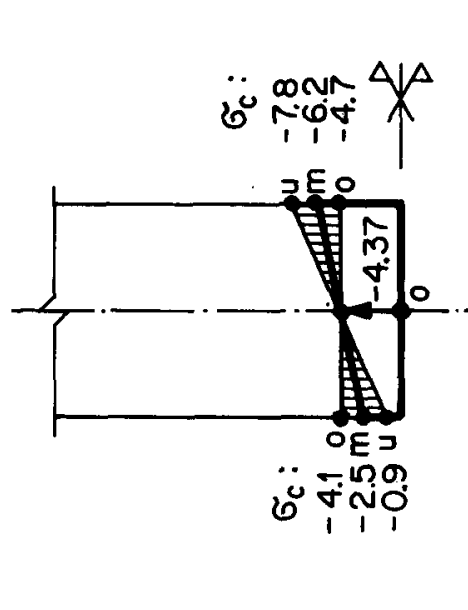 \\
\hline $\begin{array}{l}1.2 \\
f=11.23 \mathrm{~Hz} \\
w_{\text {rel, } \max }= \pm 2 \mathrm{~mm}\end{array}$ & 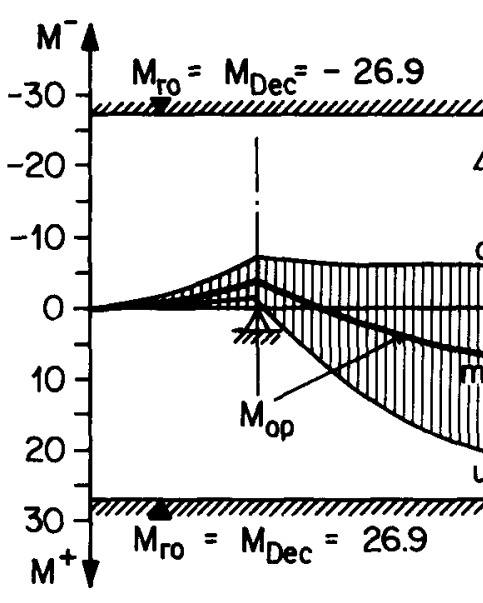 & 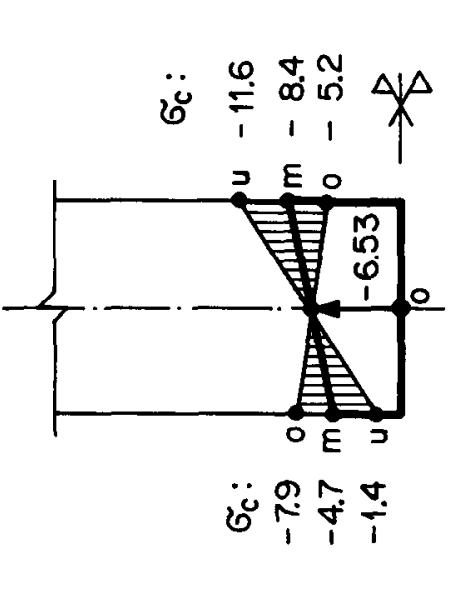 \\
\hline $\begin{array}{l}\text { (1.3) } \\
f=11.24 \mathrm{~Hz}\end{array}$ & 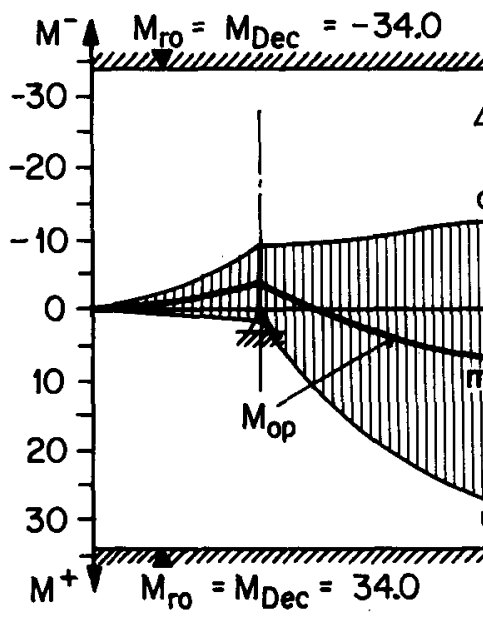 & $\ddot{0}$ \\
\hline
\end{tabular}

Annahmen: Biegesteifigkeit unabhängig von Relativdurchbiegung und konstant längs Balkenachse

Bild 21b: Verlauf der Biegebeanspruchungen in Balkenruhelage $M_{\text {op }}$ (inf. Balkeneigengewicht, Zusatzmassen, Schwinger und Vorspannung) sowie bei maximaler Relativdurchbiegung $M_{\text {rel }}$,

Phase 1, Betonbalken 


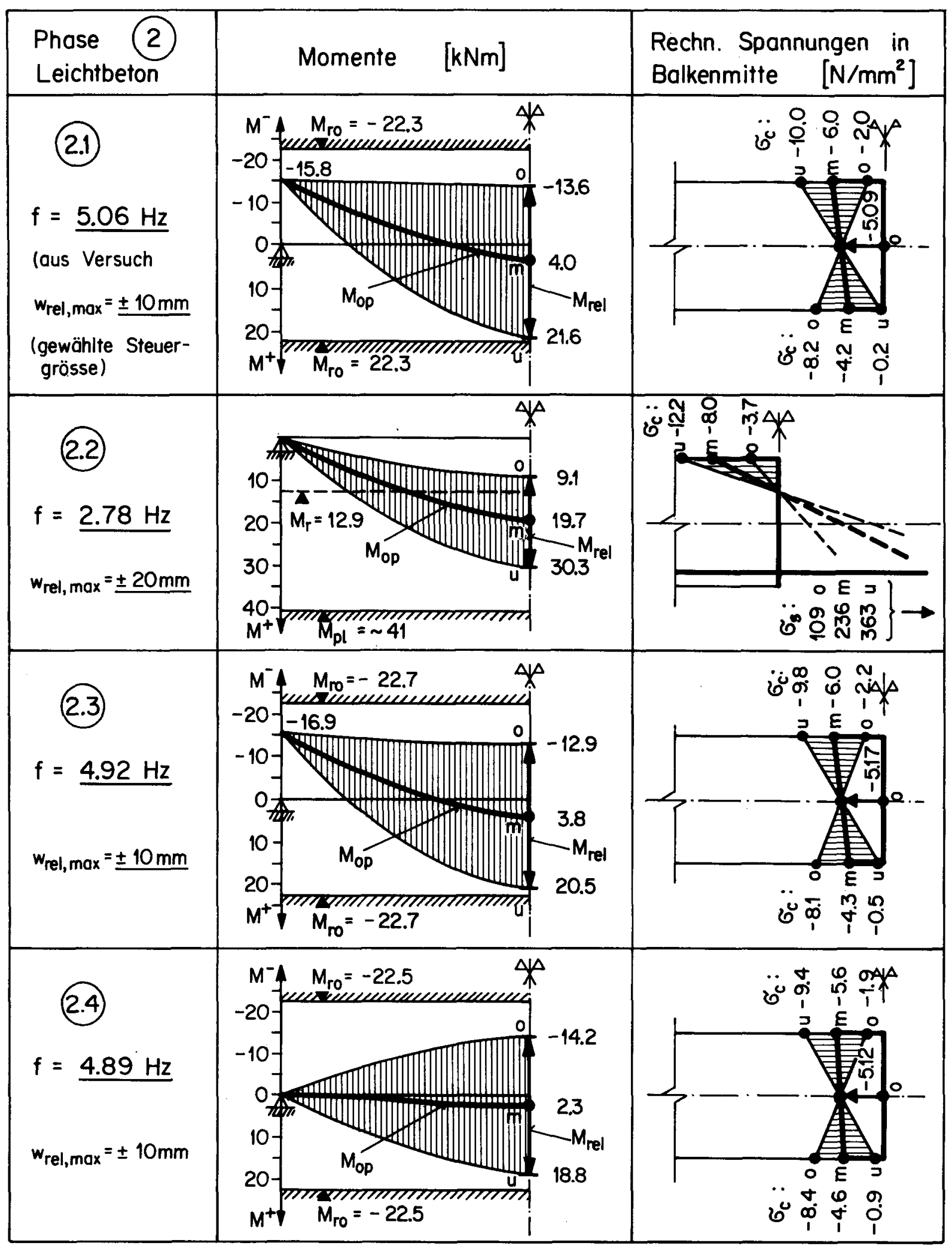

Annahmen: Biegesteifigkeit unabhängig von Relativdurchbiegung und konstant längs Balkenachse

Bild 21c : Verlauf der Biegebeanspruchungen in Balkenruhelage $M_{\text {op }}$ (inf. Balkeneigengewicht, Zusatzmassen, Schwinger und Vorspannung) sowie bei maximaler Relativdurchbiegung $M_{\text {rel }}$, Phase 2, Leichtbetonbalken 


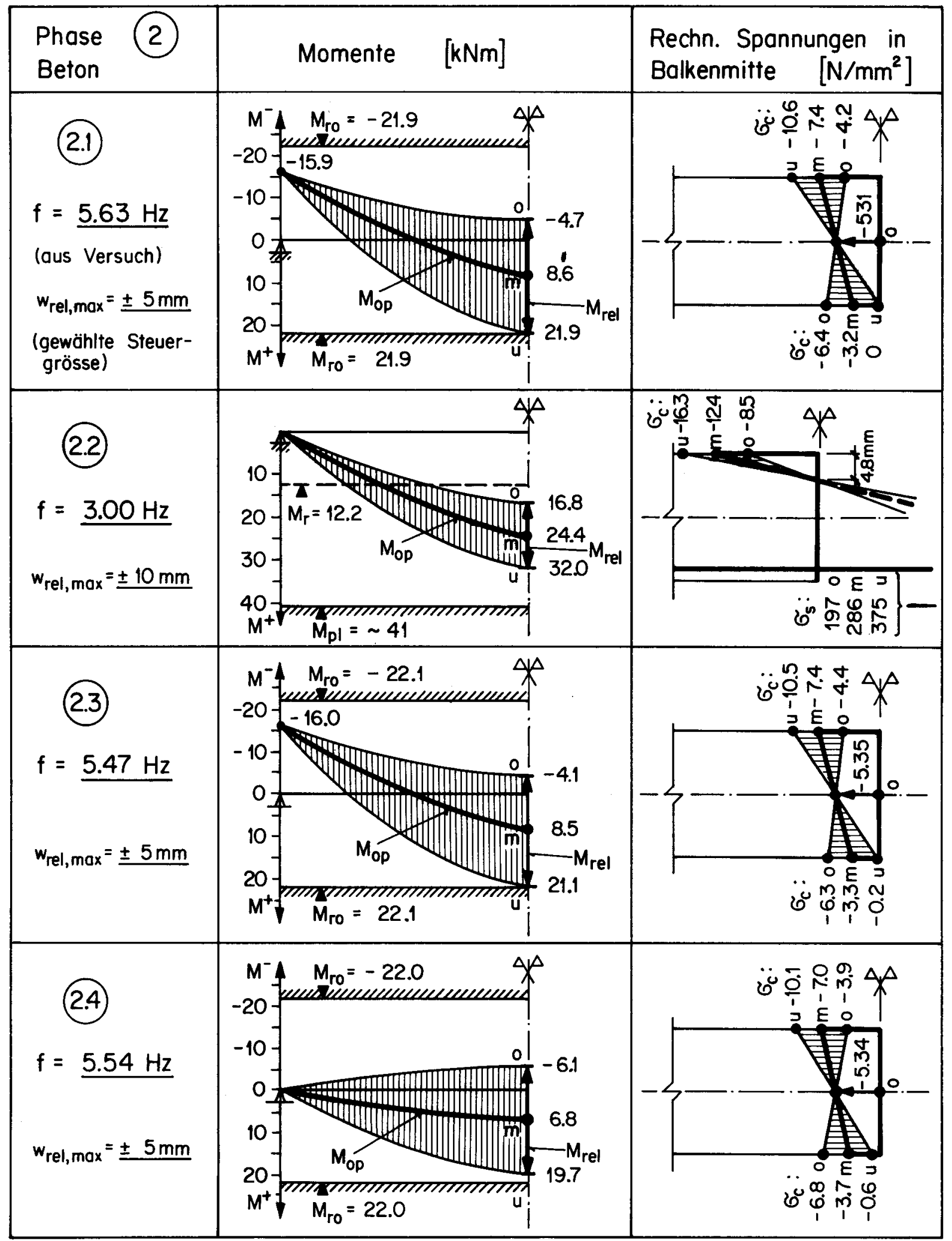

Annahmen: Biegesteifigkeit unabhängig von Relativdurchbiegung und konstant längs Balkenachse

Bild 21d: Verlauf der Biegebeanspruchungen in Balkenruhelage $M_{\text {op }}$ (inf. Balkeneigengewicht, Zusatzmassen, Schwinger und Vorspannung) sowie bei maximaler Relativdurchbiegung $M_{\text {rel }}$, Phase 2, Betonbalken 


\begin{tabular}{|c|c|c|}
\hline $\begin{array}{l}\text { Phase } \\
\text { Beton }\end{array}$ & Momente $\quad[\mathrm{kNm}]$ & $\begin{array}{l}\text { Rechn. Spannungen in } \\
\text { Balkenmitte } \quad\left[\mathrm{N} / \mathrm{mm}^{2}\right]\end{array}$ \\
\hline $\begin{array}{l}\text { (4.1) } \\
f=\frac{3.98 \mathrm{~Hz}}{\text { Versuch) }} \\
\text { (aus } \\
w_{\text {rel,max }}= \pm 20 \mathrm{~mm} \\
\text { (gewöhlte Steuer- } \\
\text { grösse) }\end{array}$ & $M_{5}=-24.7$ & (1) \\
\hline $\begin{array}{l}\text { (4.3) } \\
f=3.02 \mathrm{~Hz} \\
w_{\text {rel, } \max }= \pm 20 \mathrm{~mm}\end{array}$ & (10) & 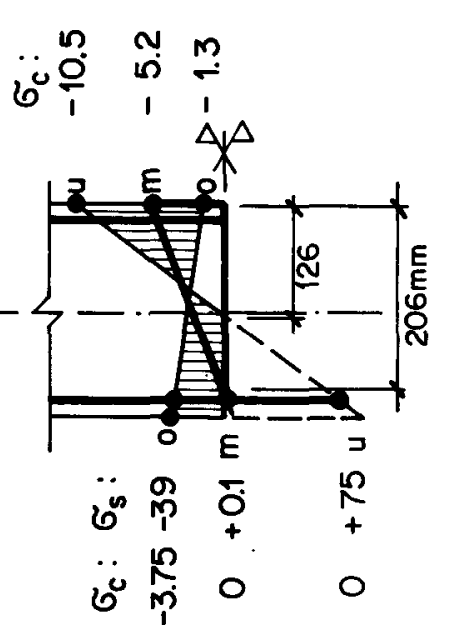 \\
\hline $\begin{array}{l}\text { (4.5) } \\
f=2.4 \mathrm{~Hz} \\
w_{\text {rel, } \text { max }}= \pm 20 \mathrm{~mm}\end{array}$ & (10) & 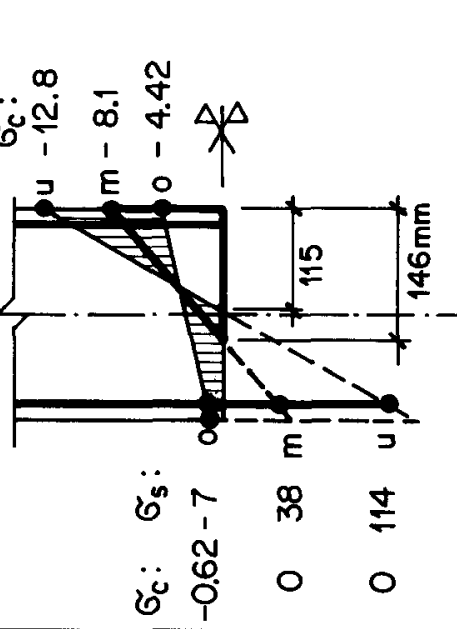 \\
\hline
\end{tabular}

Annahmen: Biegesteifigkeif unabhängig von Relativdurchbiegung und konstant längs Balkenachse

Bild 21e: Verlauf der Biegebeanspruchungen in Balkenruhelage $M_{o p}$ (inf. Balkeneigengewicht, Zusatzmassen, Schwinger und Vorspannung) sowie bei maximaler Relativdurchbiegung $M_{\text {rel }}$, Phase 4, Leichtbetonbalken (Phase 3 praktisch identisch) 


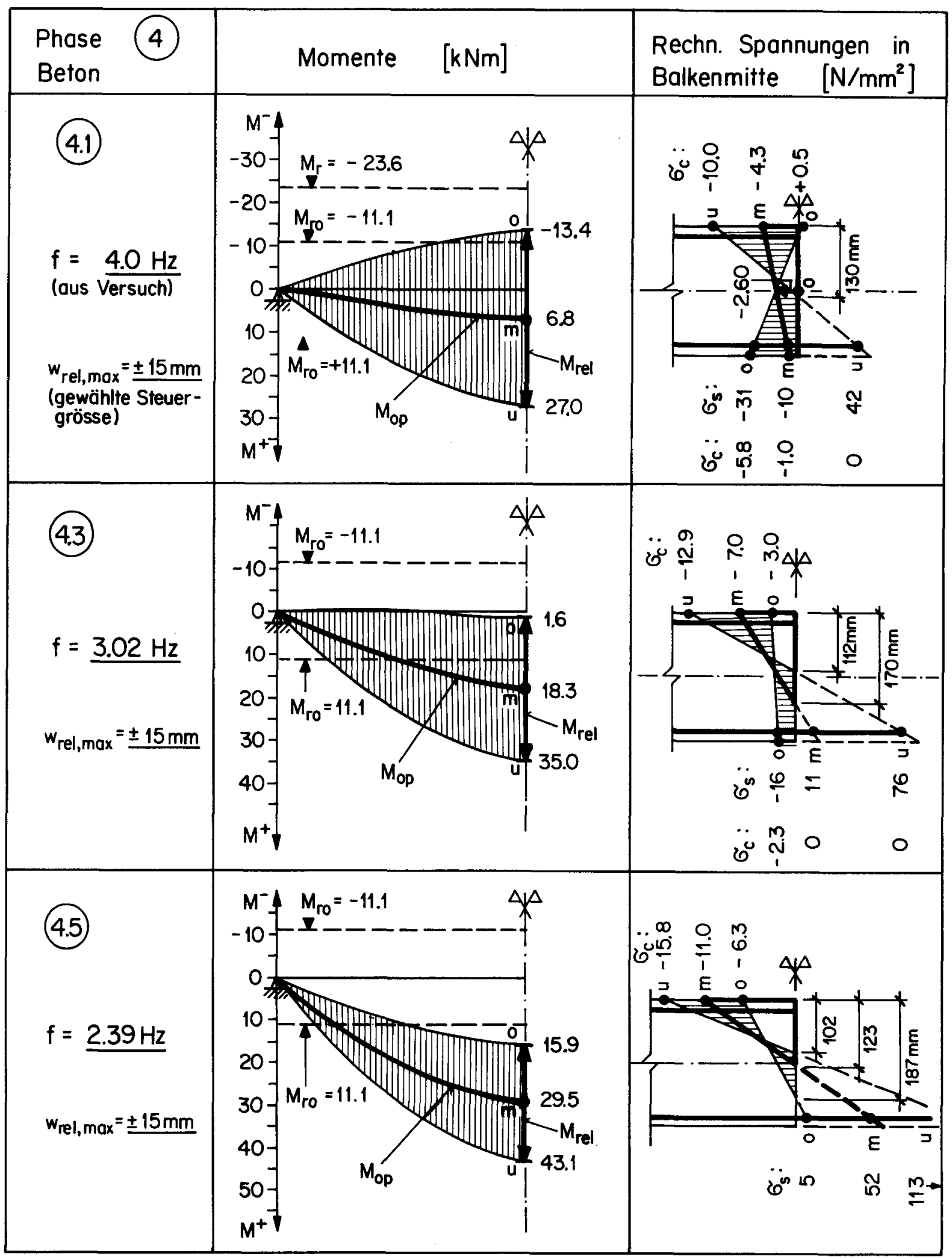

Annahmen: Biegesteifigkeit unabhängig von Relativdurchbiegung und konstant längs Balkenachse

Bild 21f: Verlauf der Biegebeanspruchungen in Balkenruhelage $M_{\text {op }}$ (inf. Balkeneigengewicht, Zusatzmassen, Schwinger und Vorspannung) sowie bei maximaler Relativdurchbiegung $M_{\text {rel }}$, Phase 4, Leichtbetonbalken (Phase 3 praktisch identisch) 

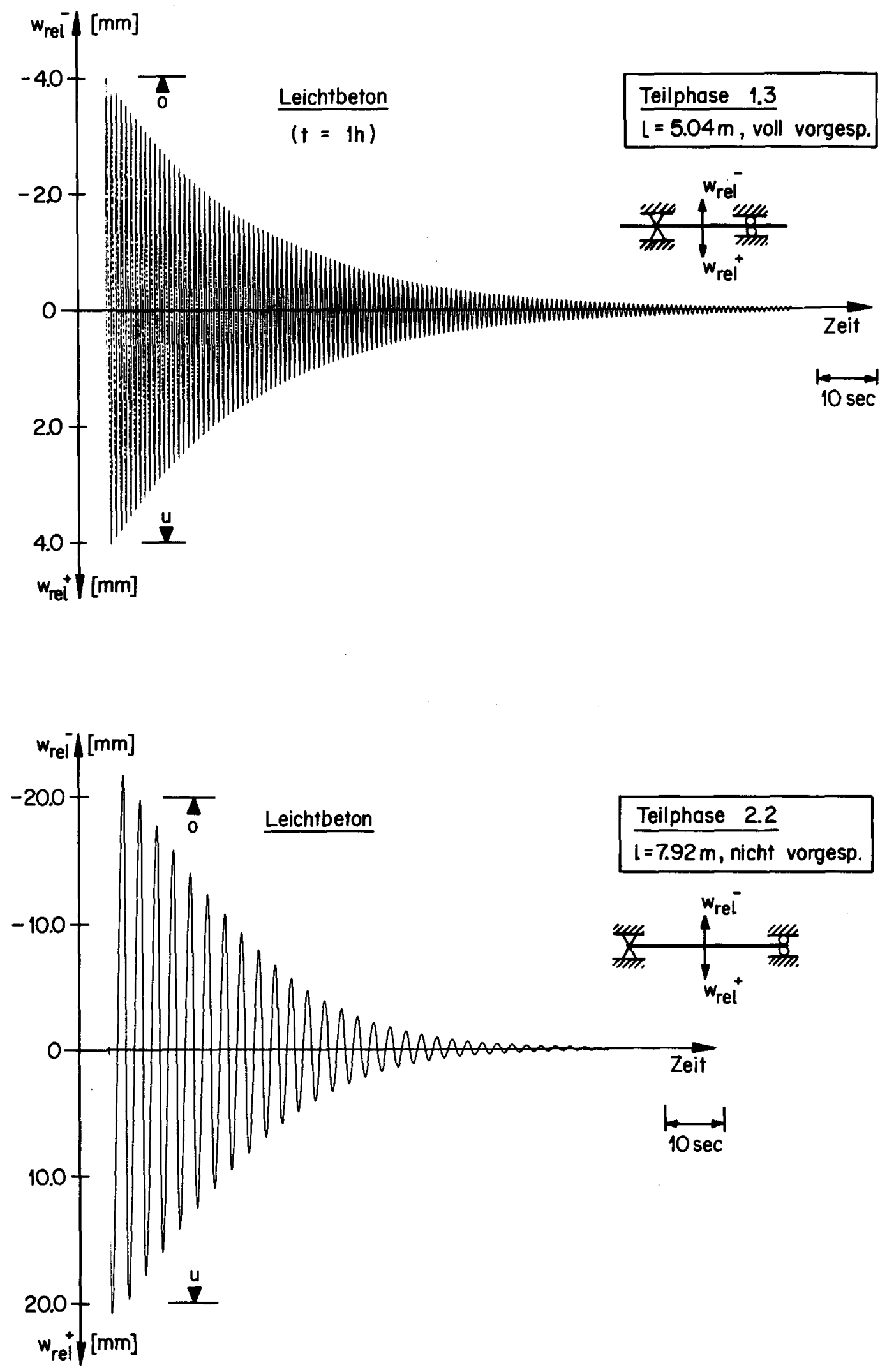

Bild 22a: Ausschwingkurven der Relativdurchbiegungen in Balkenmitte, Teilphasen 1.3, 2.2, Leichtbetonbalken 
$-65-$
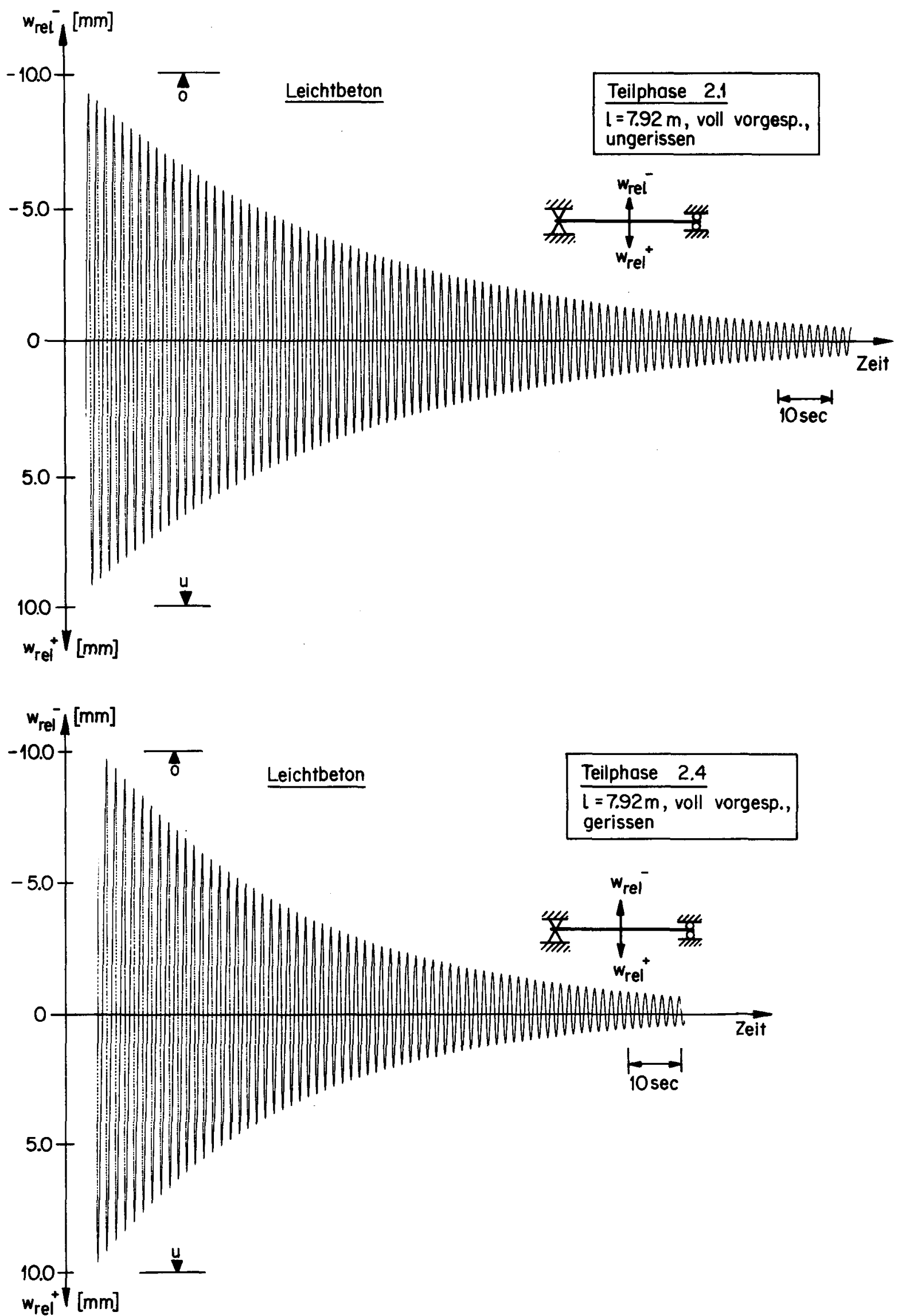

Bild 22b: Ausschwingkurven der Relativdurchbiegungen in Balkenmitte, Teilphasen $2.1,2.4$, Leichtbetonbalken 


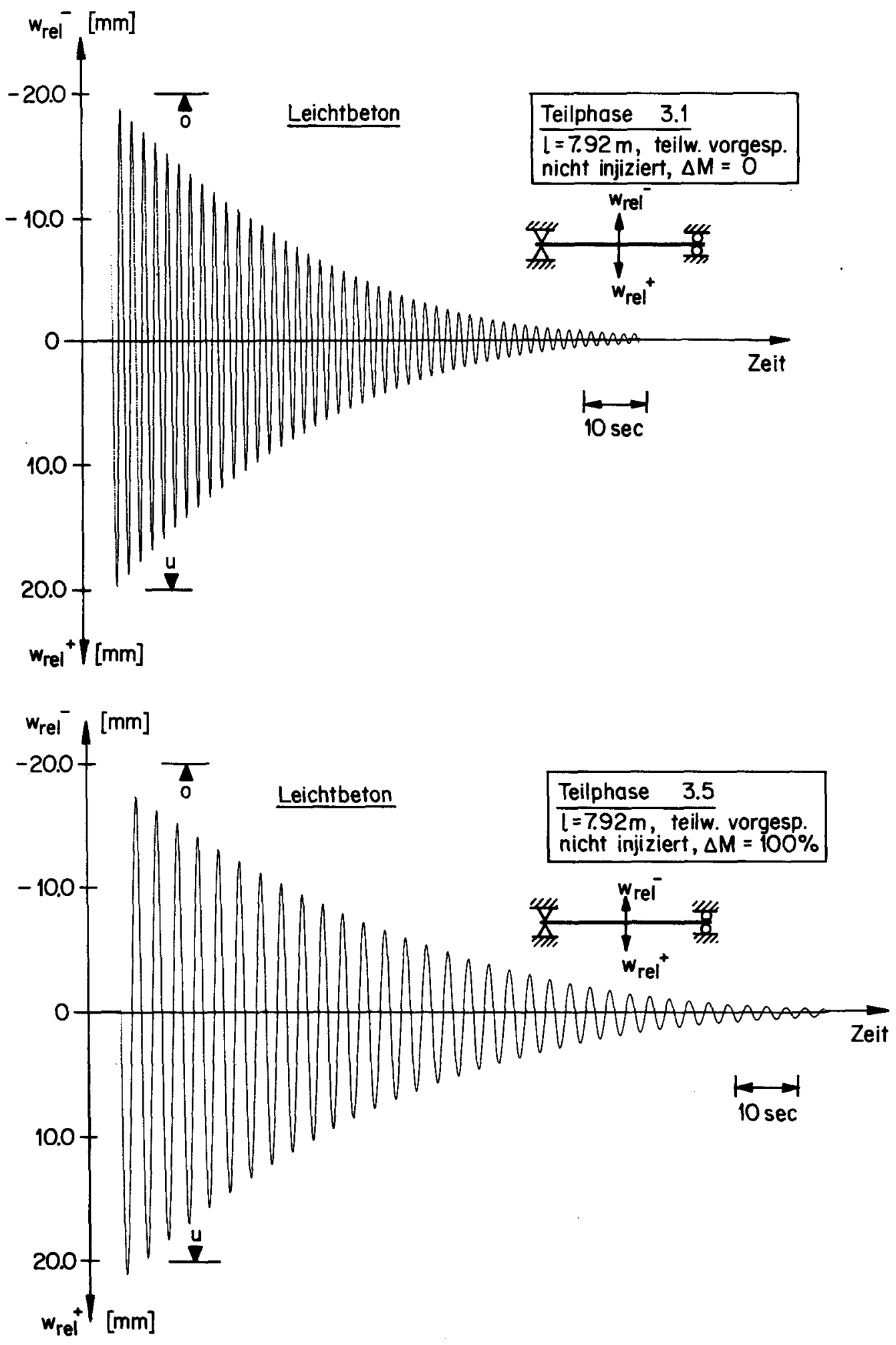

Bild 22c: Ausschwingkurven der Relativdurchbiegungen in Balkenmitte, Teilphasen $3.1,3.5$, Leichtbetonbalken 


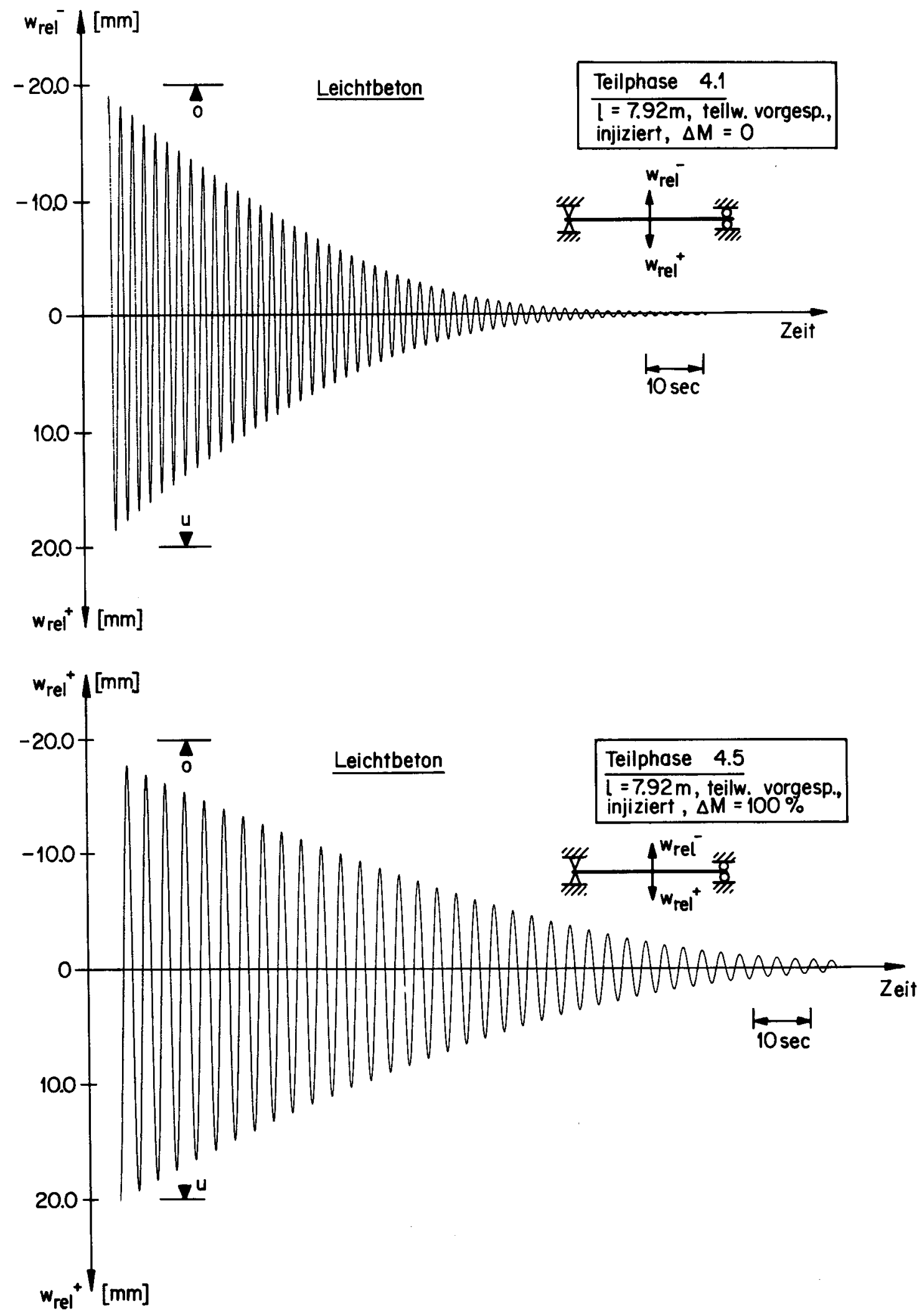

Bild 22d: Ausschwingkurven der Relativdurchbiegungen in Balkenmitte, Teilphase 4.1, 4.5, Leichtbetonbalken 


\section{Betonbalken}

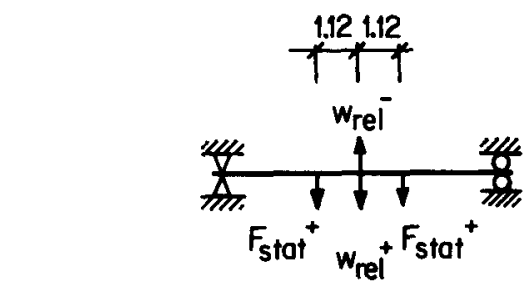

Last - Durchbiegung

Übersicht

$$
F_{\text {stat }}+[k N] \uparrow \text { Zug pro Kolben }
$$

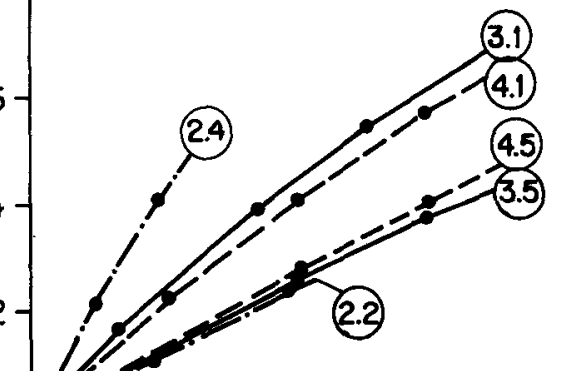

$w_{\text {rei }}[\mathrm{mm}] \quad-20 \quad-16 \quad-12 \quad-8 \quad-4$

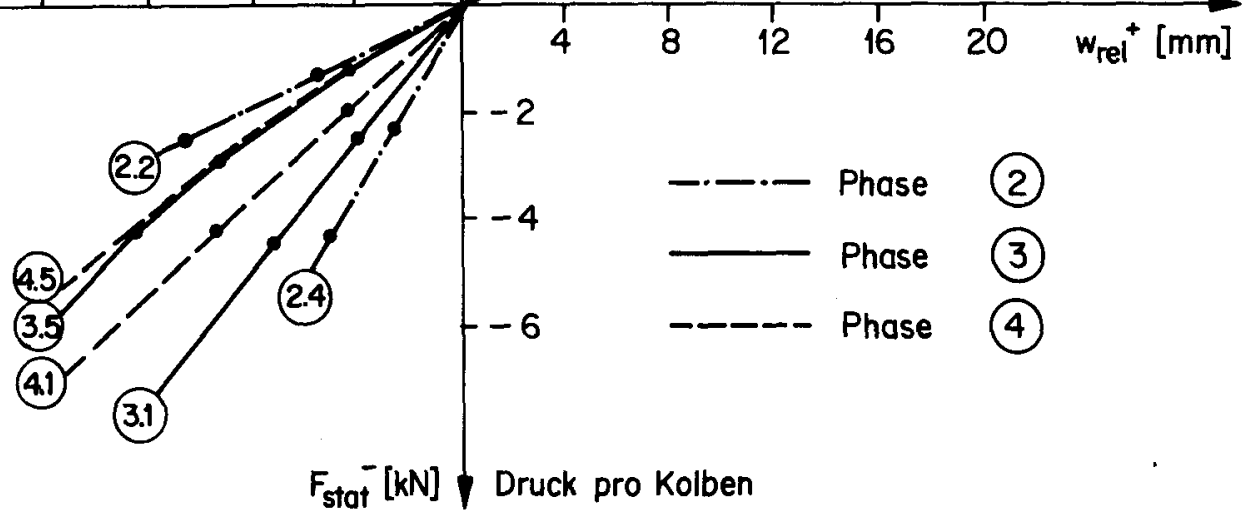

Leichtbetonbalken

$$
\mathrm{F}_{\text {stat }}^{+}[\mathrm{kN}] \uparrow \text { Zug pro Kolben }
$$
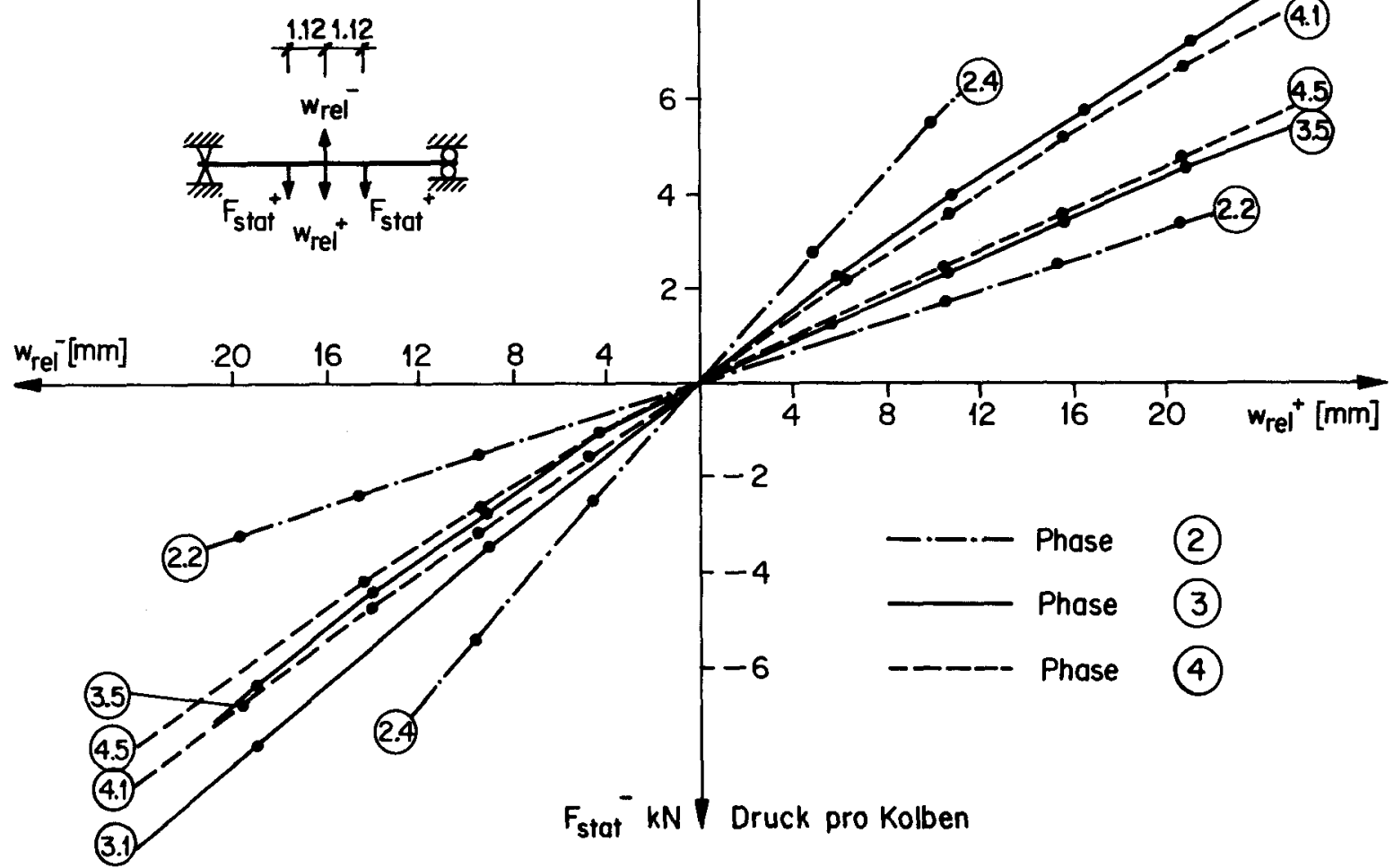

Bild 23a : Übersicht des Last - Durchbiegungsverhaltens beim statischen Belastungsversuch 


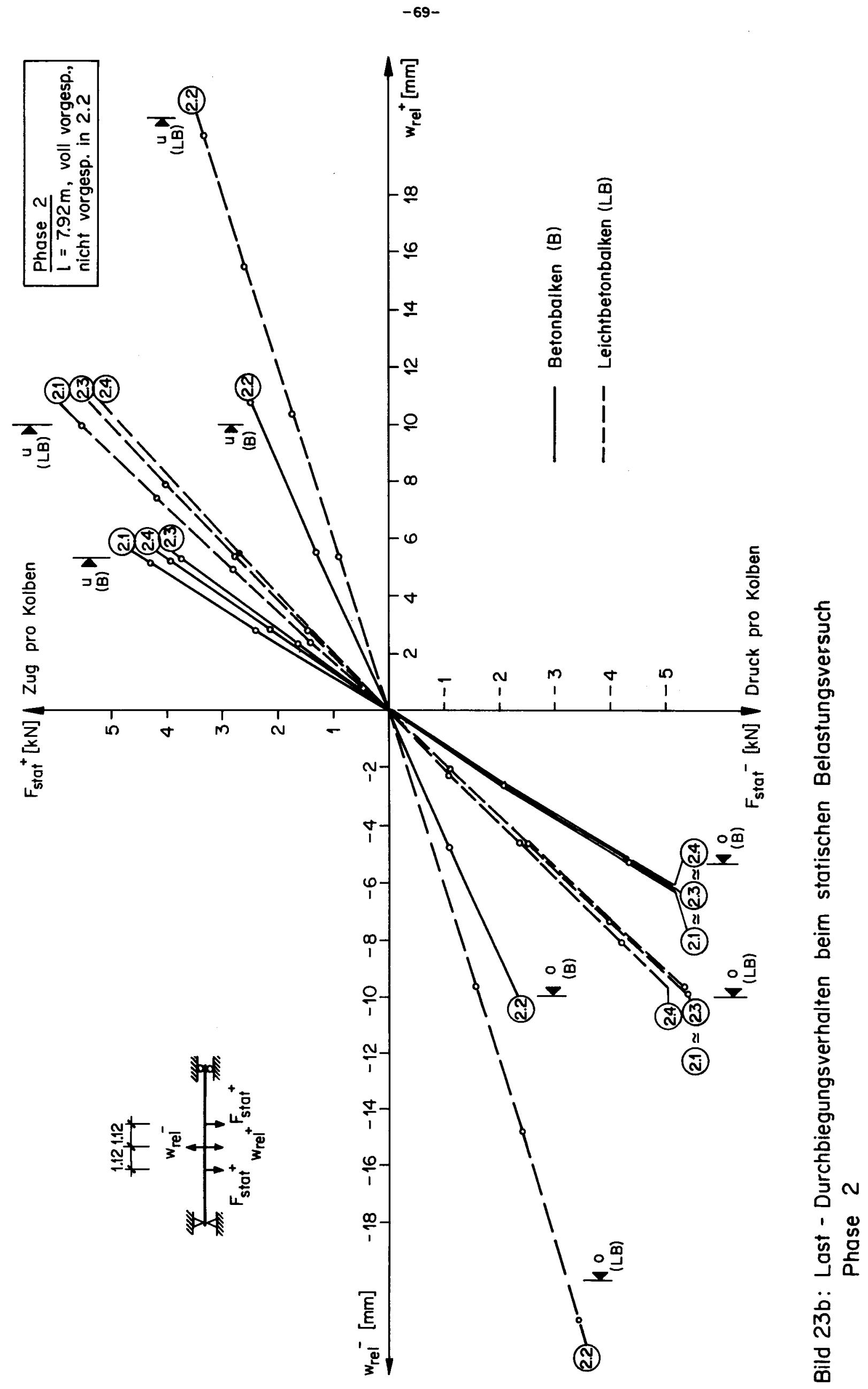




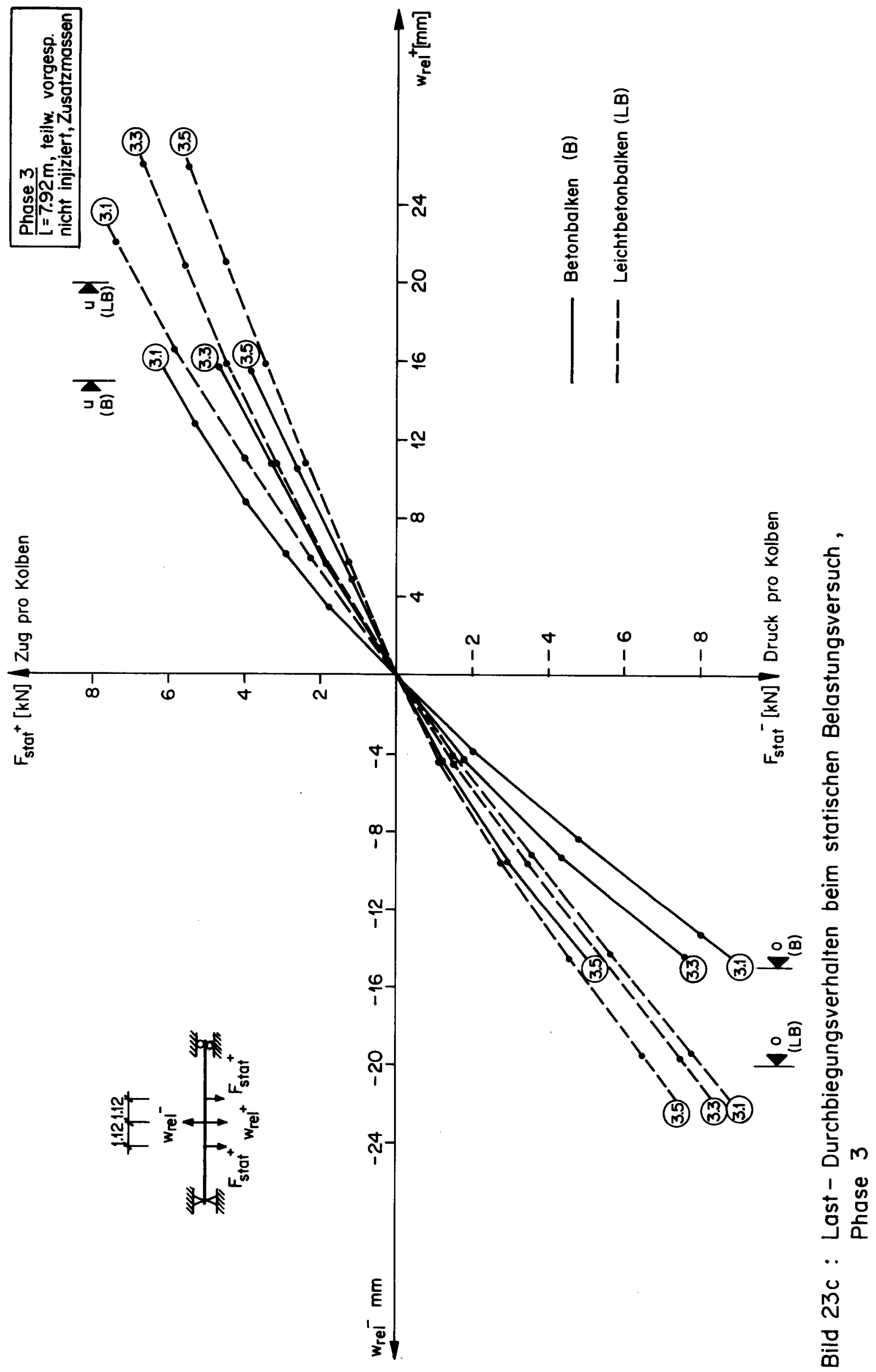




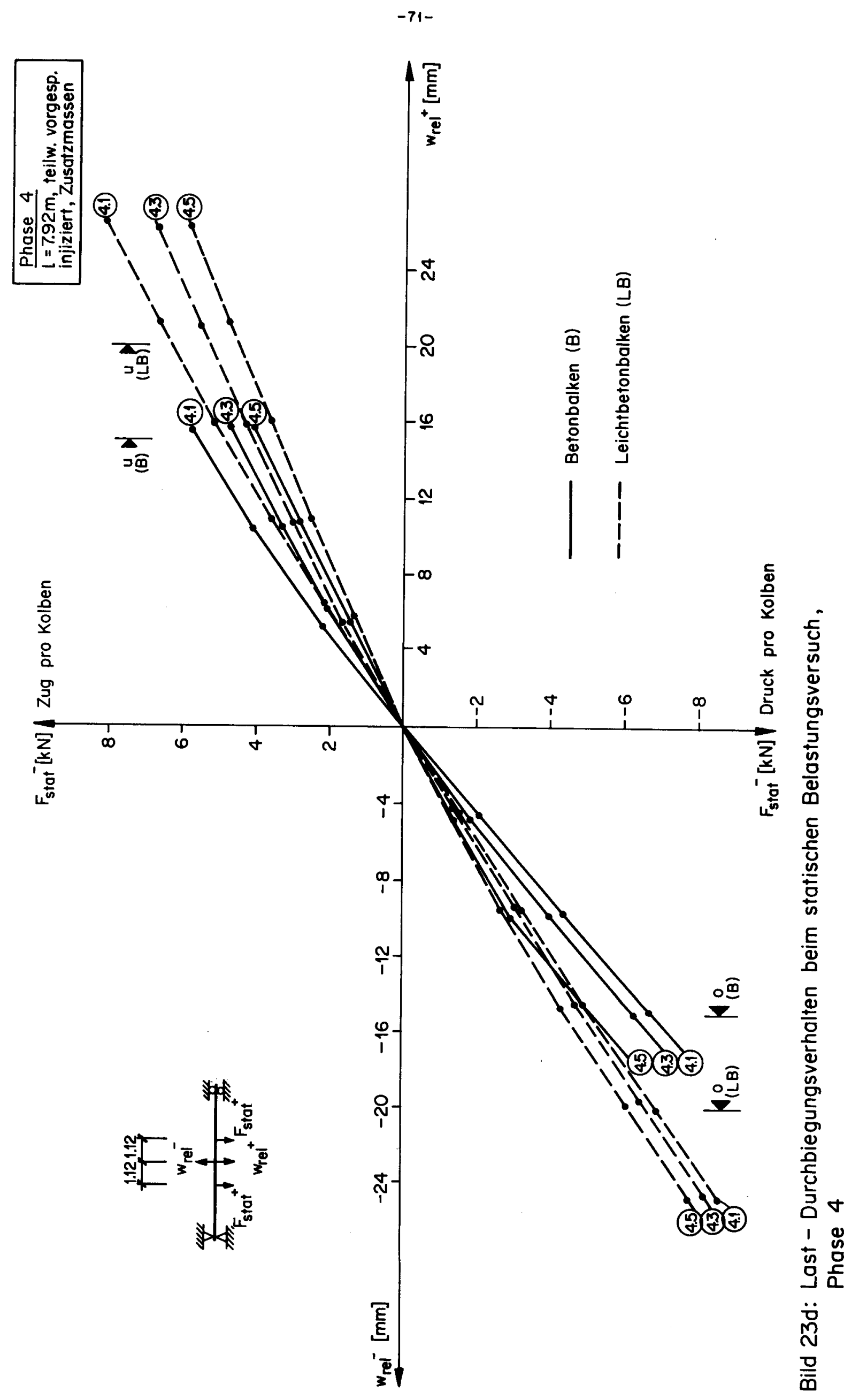




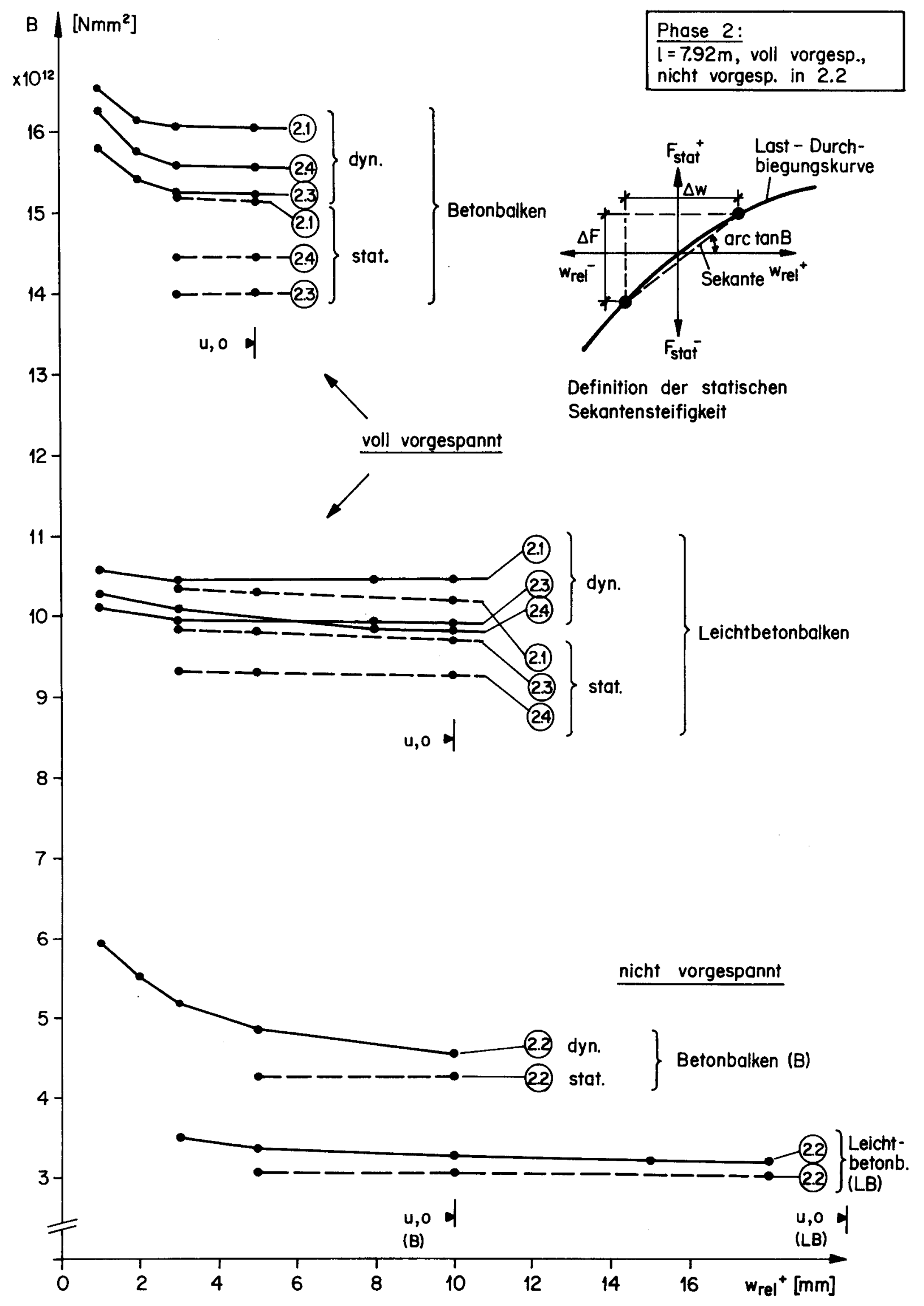

Bild 24a: Dynamische Biegesteifigkeit und statische Sekantensteifigkeit in Funktion der Relativdurchbiegung nach unten $w_{\text {rel }}{ }^{+}$in Balkenmitte, Phase 2 
$-73-$
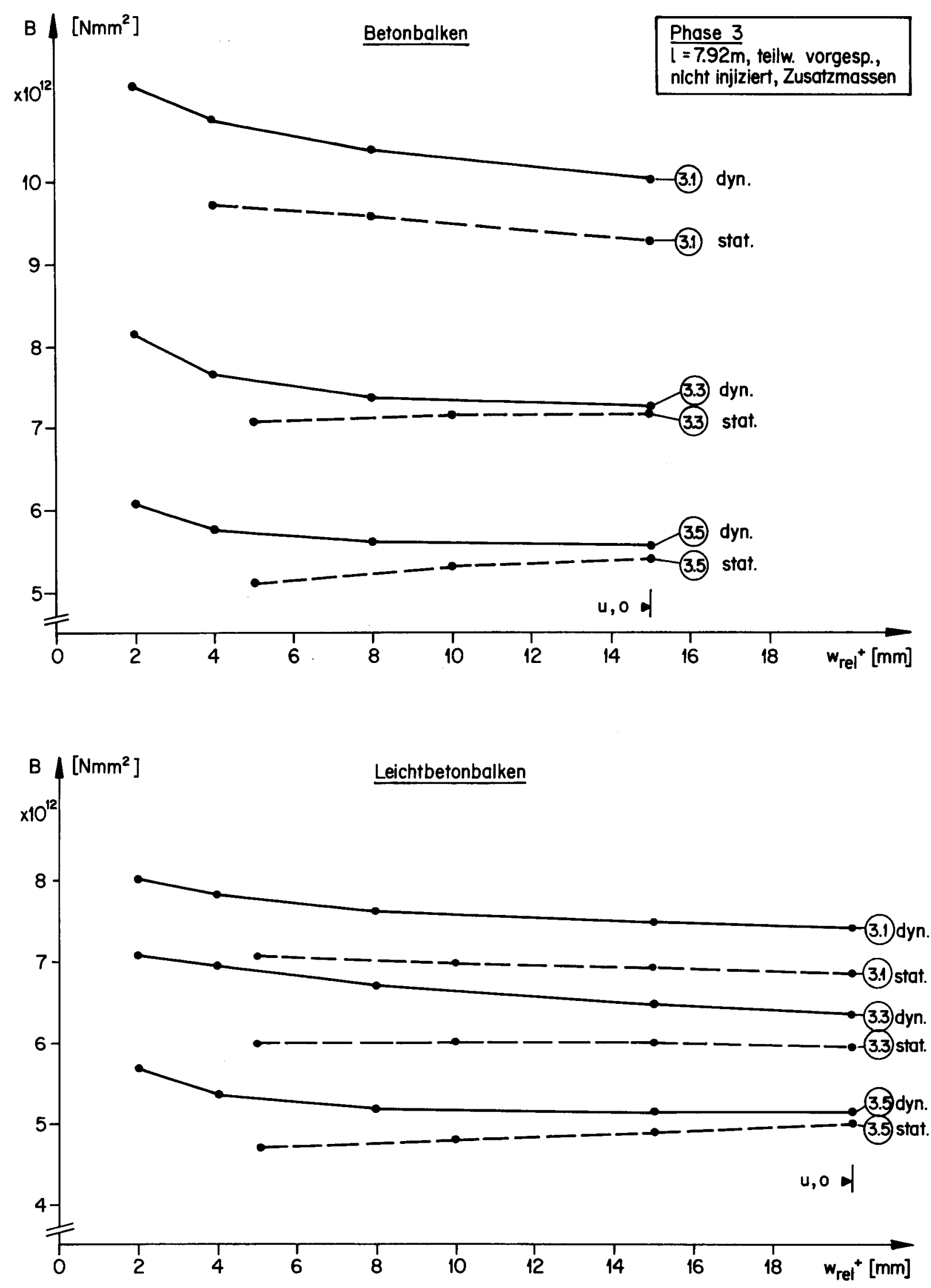

Bild 24b: Dynamische Biegesteifigkeit und statische Sekantensteifigkeit in Funktion der Relativdurchbiegung nach unten $\mathrm{w}_{\text {rel }}{ }^{+}$in Balkenmitte, Phase 3 

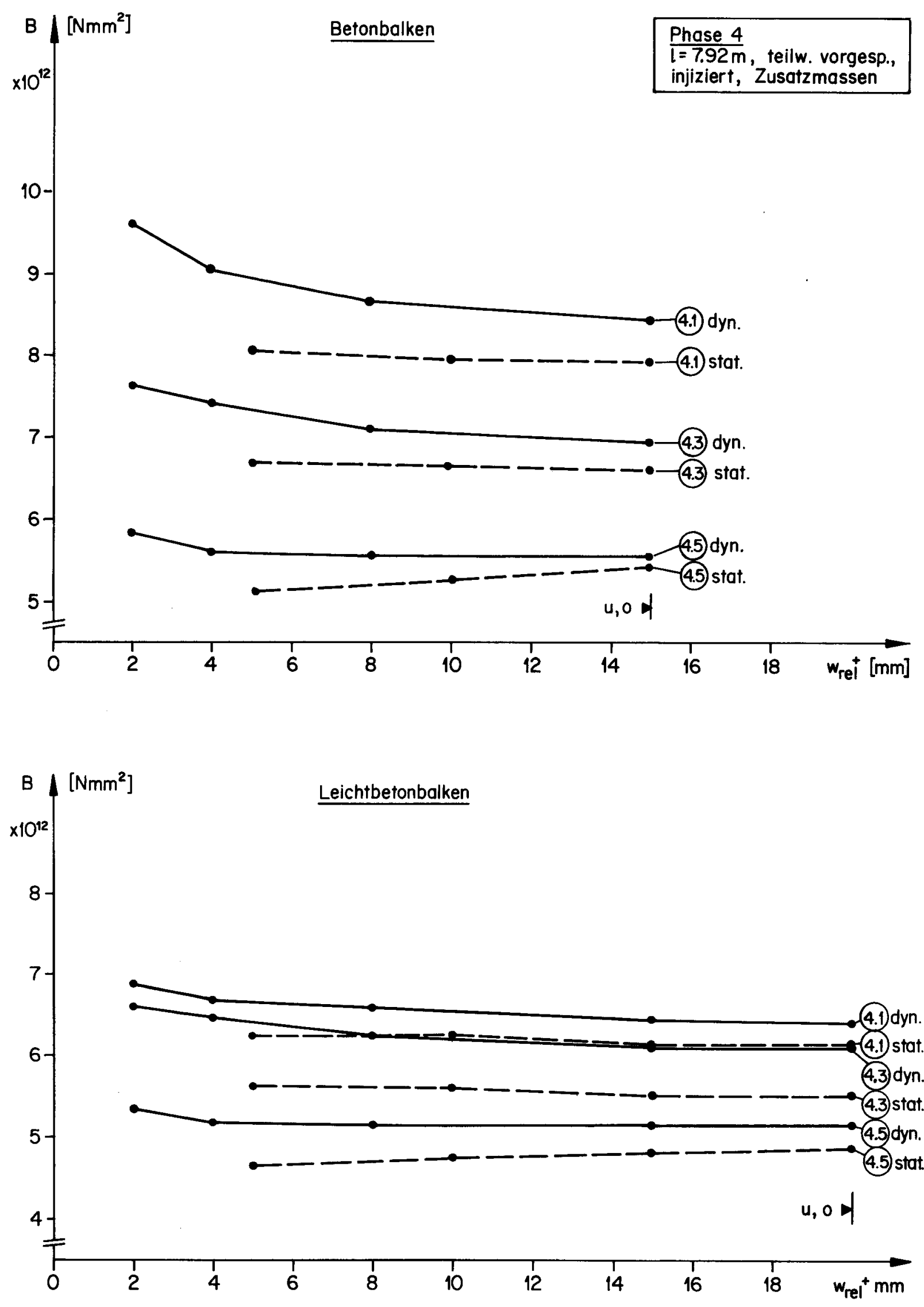

Bild 24c: Dynamische Biegesteifigkeit und statische Sekantensteifigkeit in Funktion der Relativdurchbiegung nach unten $\mathrm{w}_{\mathrm{rel}}{ }^{+}$in Balkenmitte, Phase 4 


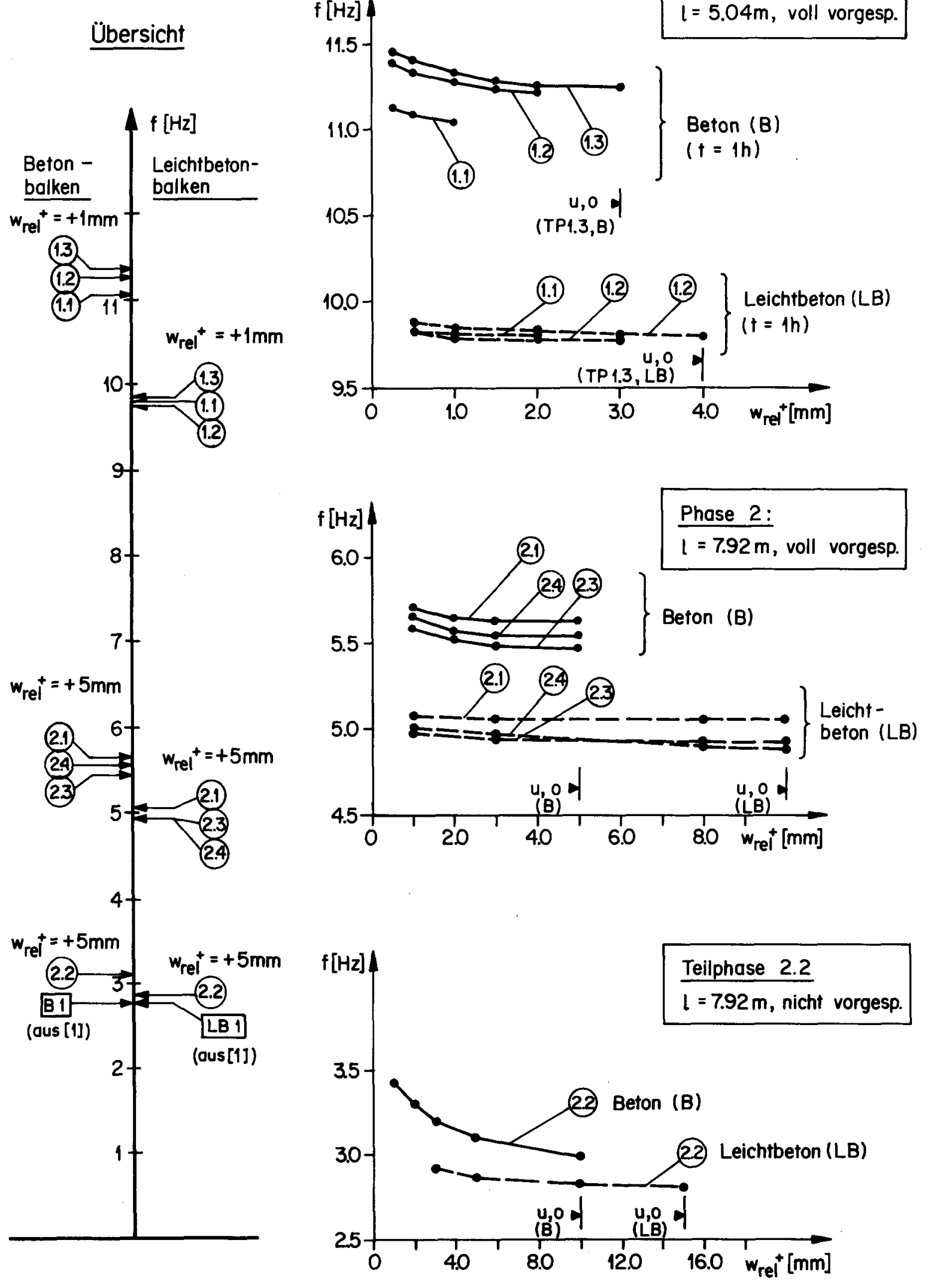

Bild 25a: Eigenfrequenzen in Funktion der Relativdurchbiegung nach unten $\mathrm{wrel}^{+}$in Balkenmitte, Phasen 1 und 2 


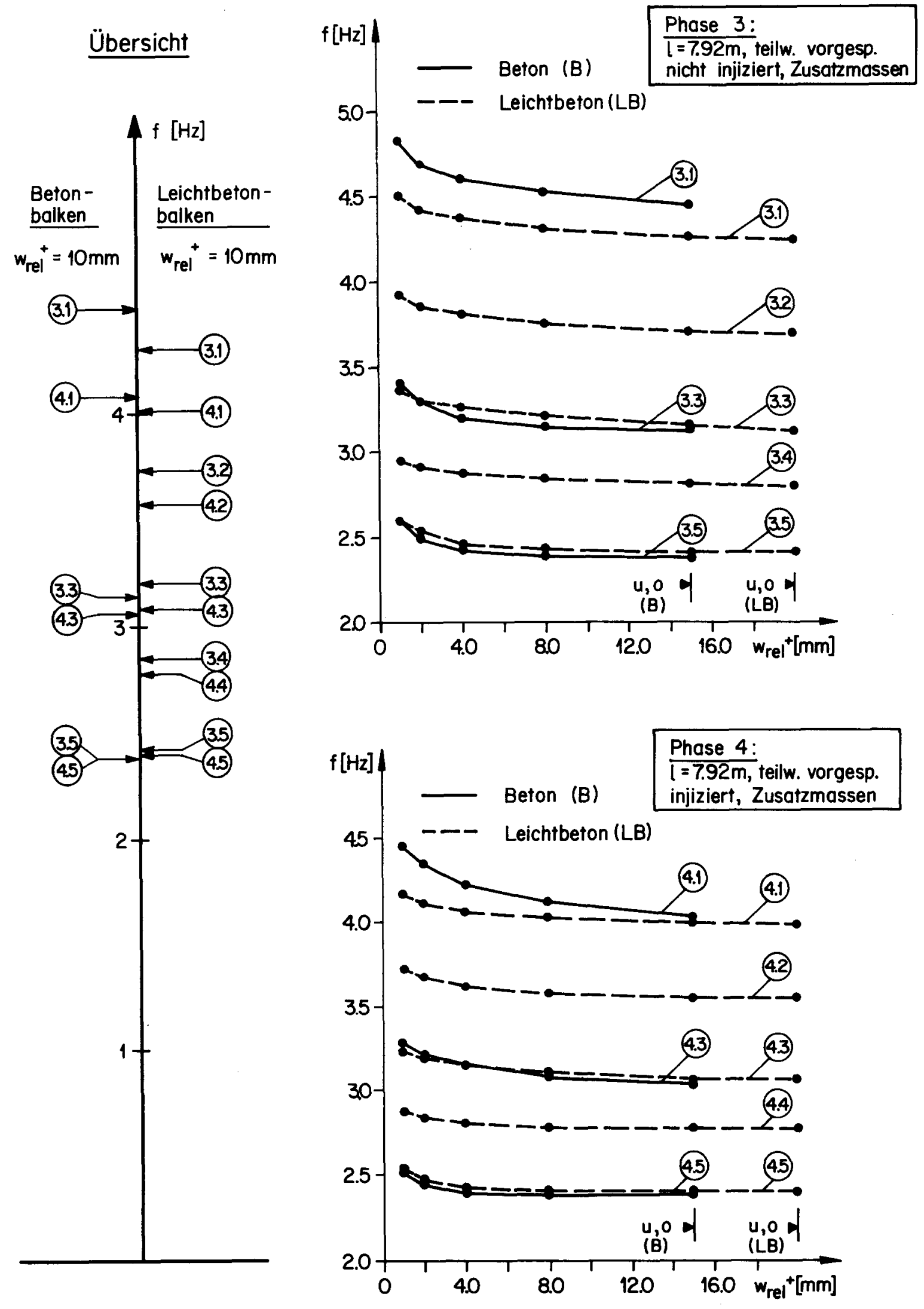

Bild 25b : Eigenfrequenzen in Funktion der Relativdurchbiegung nach unten $\mathrm{w}_{\text {rel }}{ }^{+}$in Balkenmitte, Phasen 3 und 4 

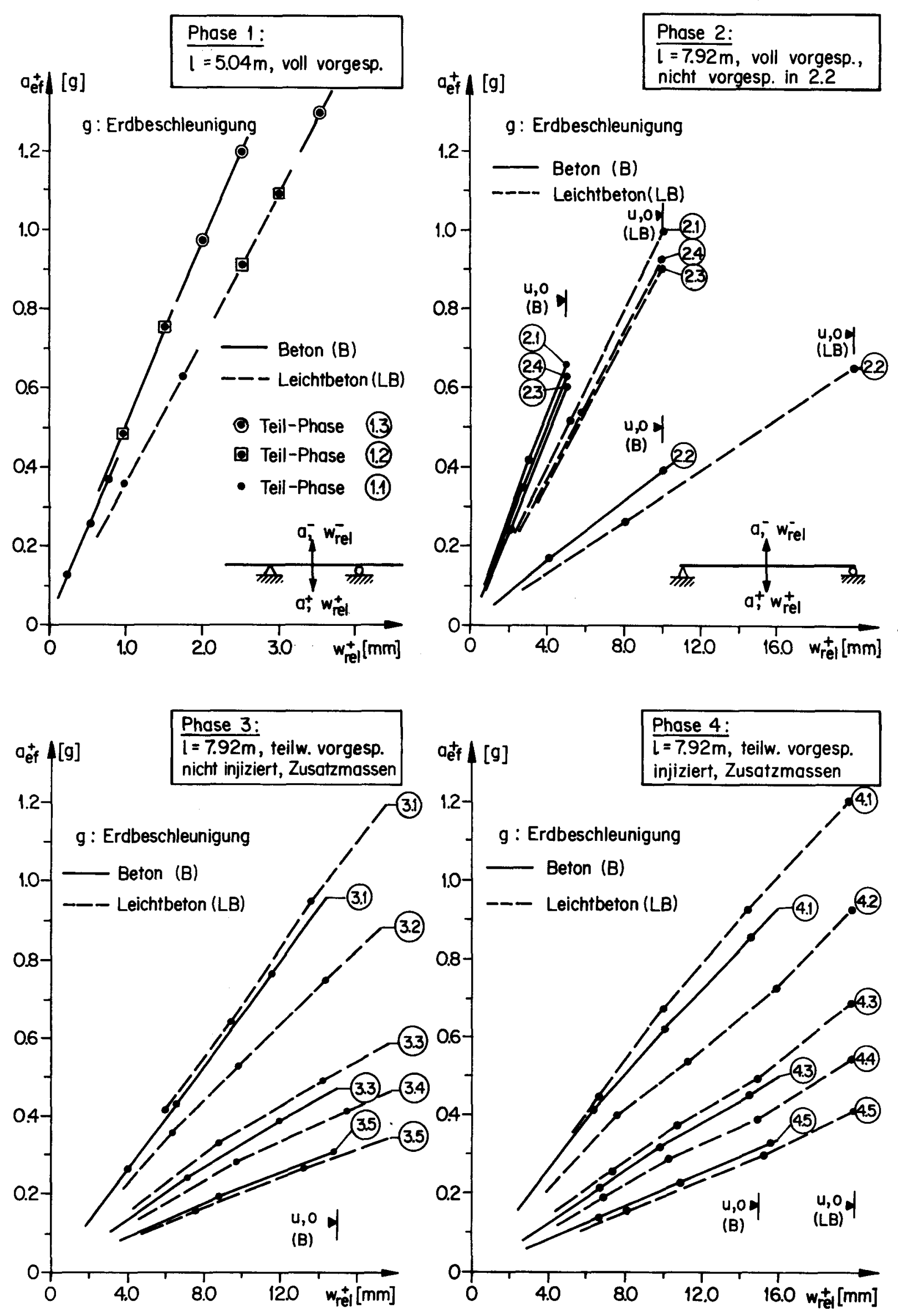

Bild 26 : Gemessene Beschleunigung $a_{\text {ef }}{ }^{+}$in Balkenmitte in Funktion der Relativ durchbiegung nach unten $w_{\text {rel }}{ }^{+}$beim Ausschwingversuch 

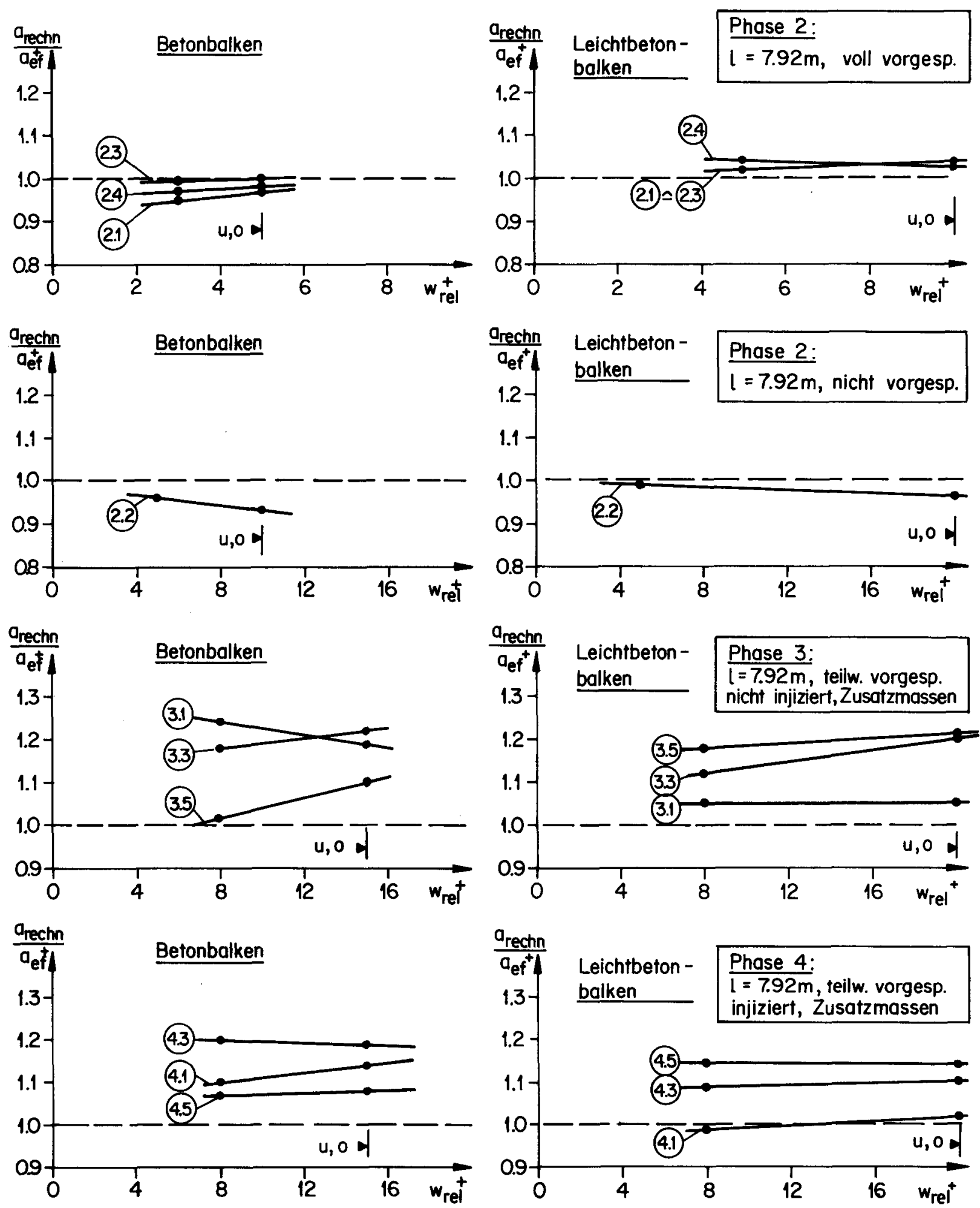

Annahmen : Biegesteifigkeit unabhängig von Durchbiegung und konstant längs Balkenachse

Bild 27 : Verhältnis der rechnerischen Beschleunigung $a_{\text {rechn }}$ zur gemessenen Beschleunigung $a_{\text {ef }}^{+}$für Relativdurchbiegung nach unten $w_{\text {rel }}^{+}$in Balkenmitte 

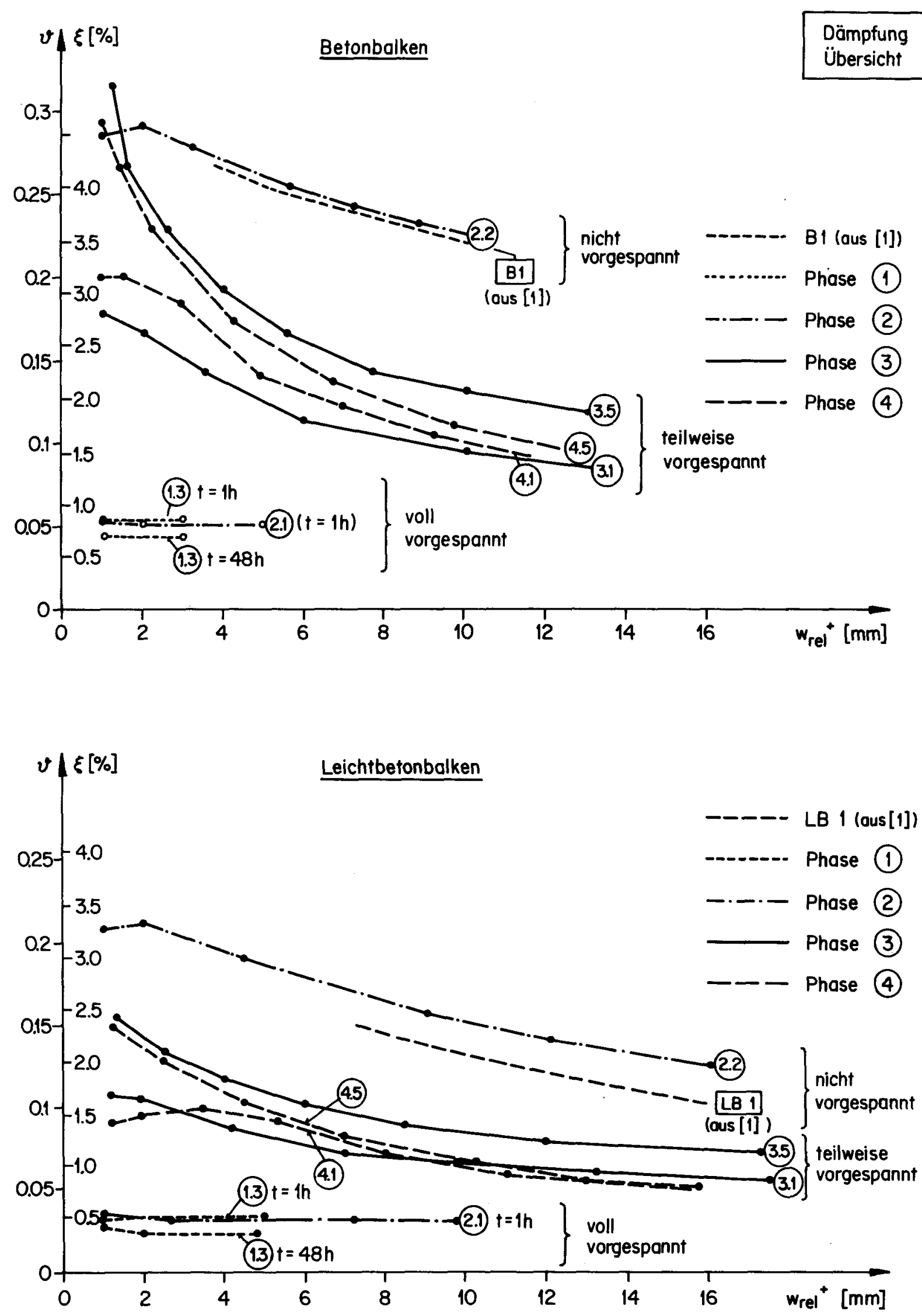

Bild 28a: Übersicht Dämpfung (logarithmisches Dekrement $v$ bzw. Dämpfungsmass $\xi$ ) in Funktion der Relativdurchbiegung nach unten $w_{\text {rel }}{ }^{+}$in Balkenmitte 

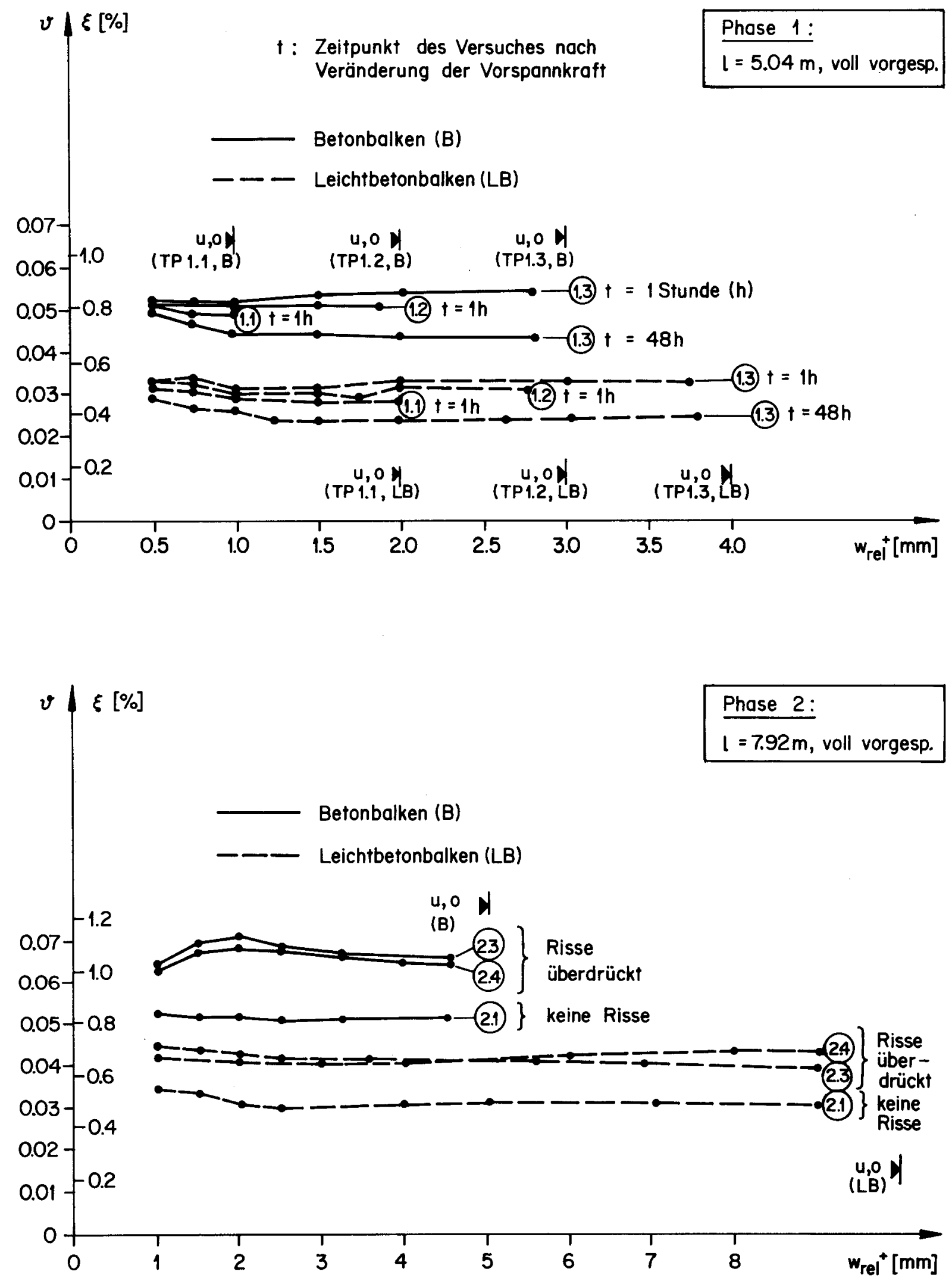

Bild 28b: Dämpfung (logarithmisches Dekrement $v$ bzw. Dämpfungsmass $\xi$ ) in Funktion der Relativdurchbiegung nach unten $w_{\text {rel }}{ }^{+}$in Balkenmitte, Phase 1 und Teilphasen $2.1,2.3,2.4$ 

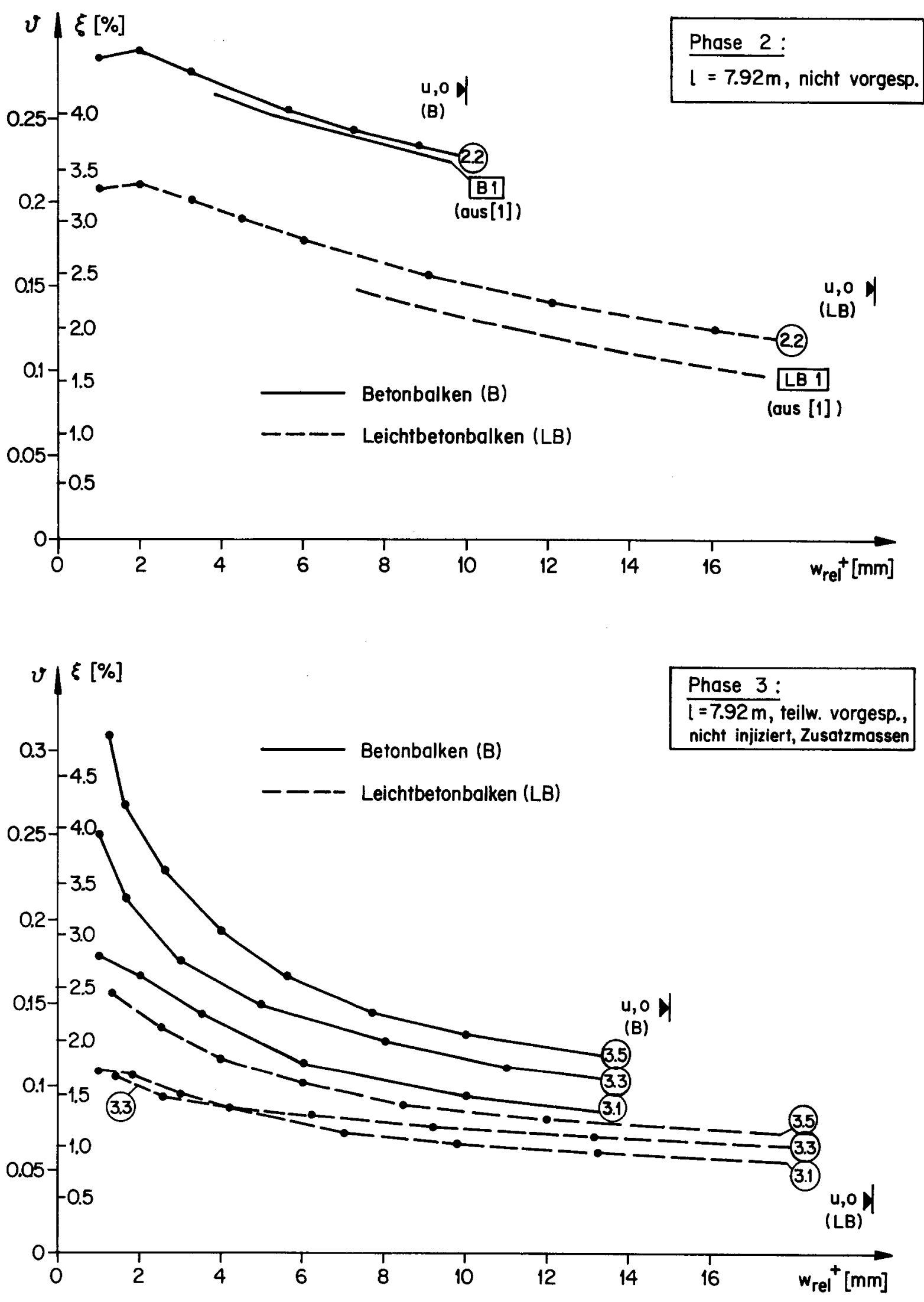

Bild 28c : Dämpfung (logarithmisches Dekrement $v$ bzw. Dämpfungsmass $\xi$ ) in Funktion der Relativdurchbiegung nach unten $\mathrm{wrel}_{\text {rel }}{ }^{+}$in Balkenmitte, Teilphase 2.2 und Phase 3 


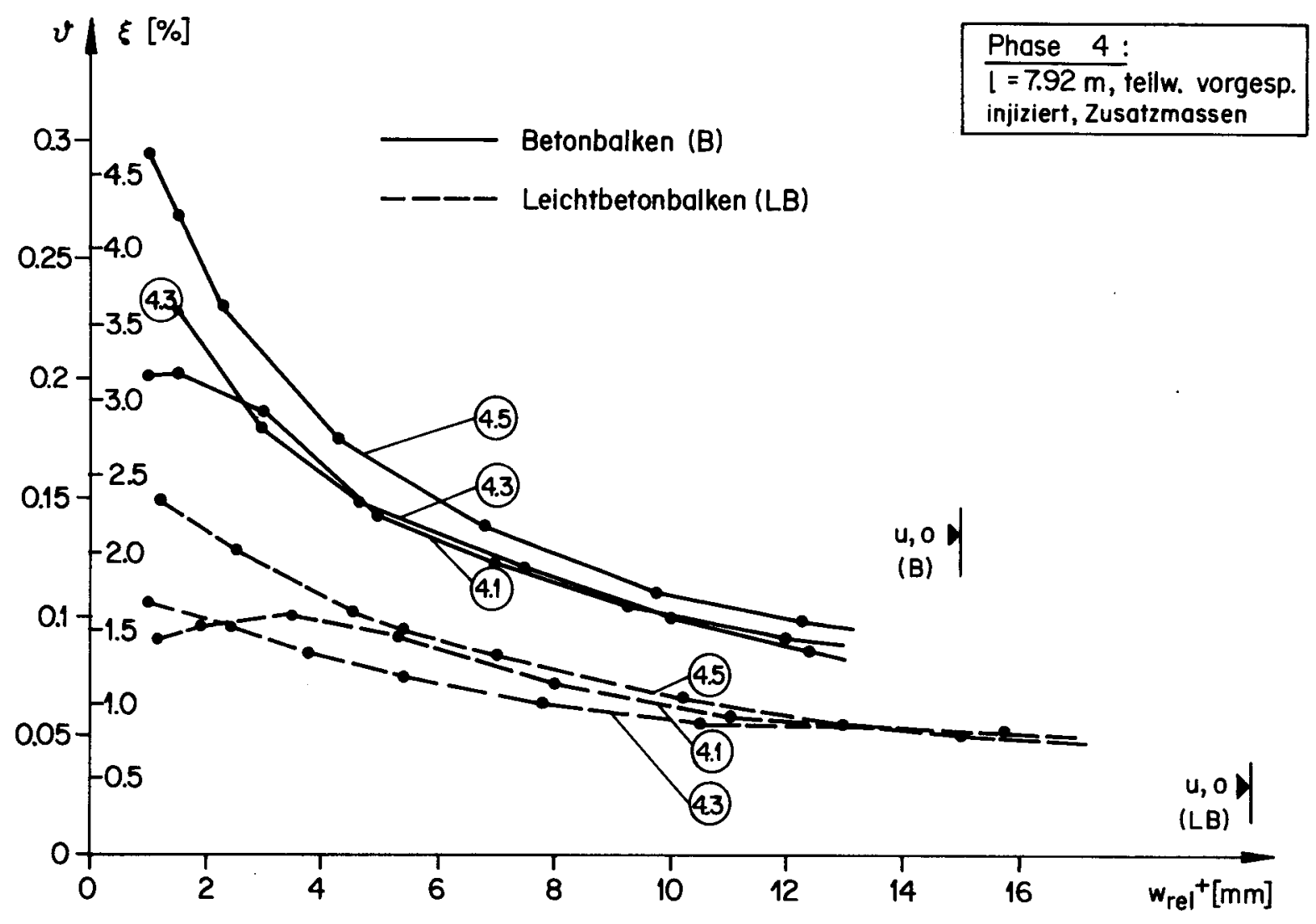

Bild 28d: Dämpfung (logarithmisches Dekrement $v$ bzw. Dämpfungsmass $\xi$ ) in Funktion der Relativdurchbiegung nach unten $\mathrm{w}_{\text {rel }}{ }^{+}$in Balkenmitte, Phase 4

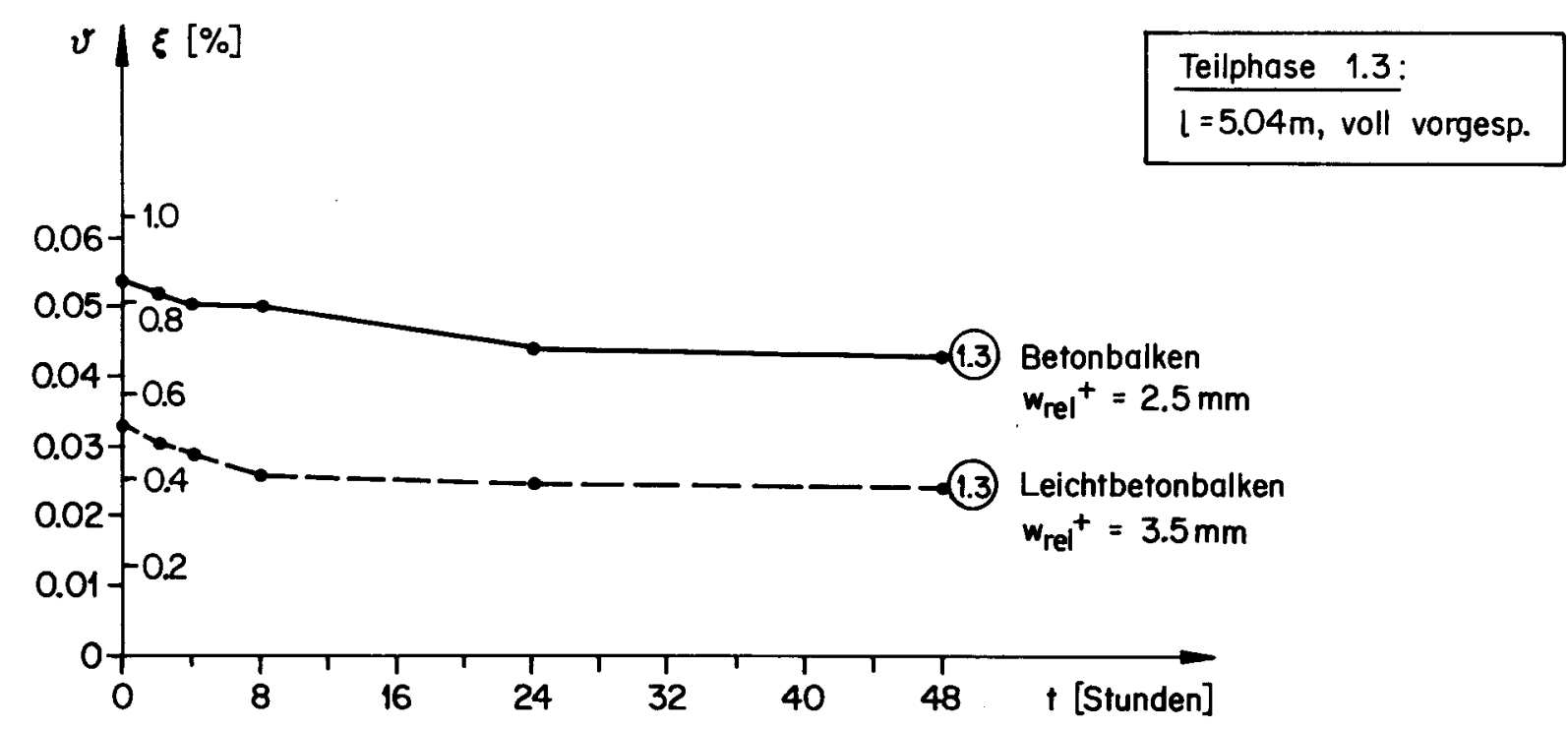

Bild 29 : Dämpfung in Funktion des Versuchszeitpunktes nach Veränderung der Vorspannkraft, Teilphase 1.3 

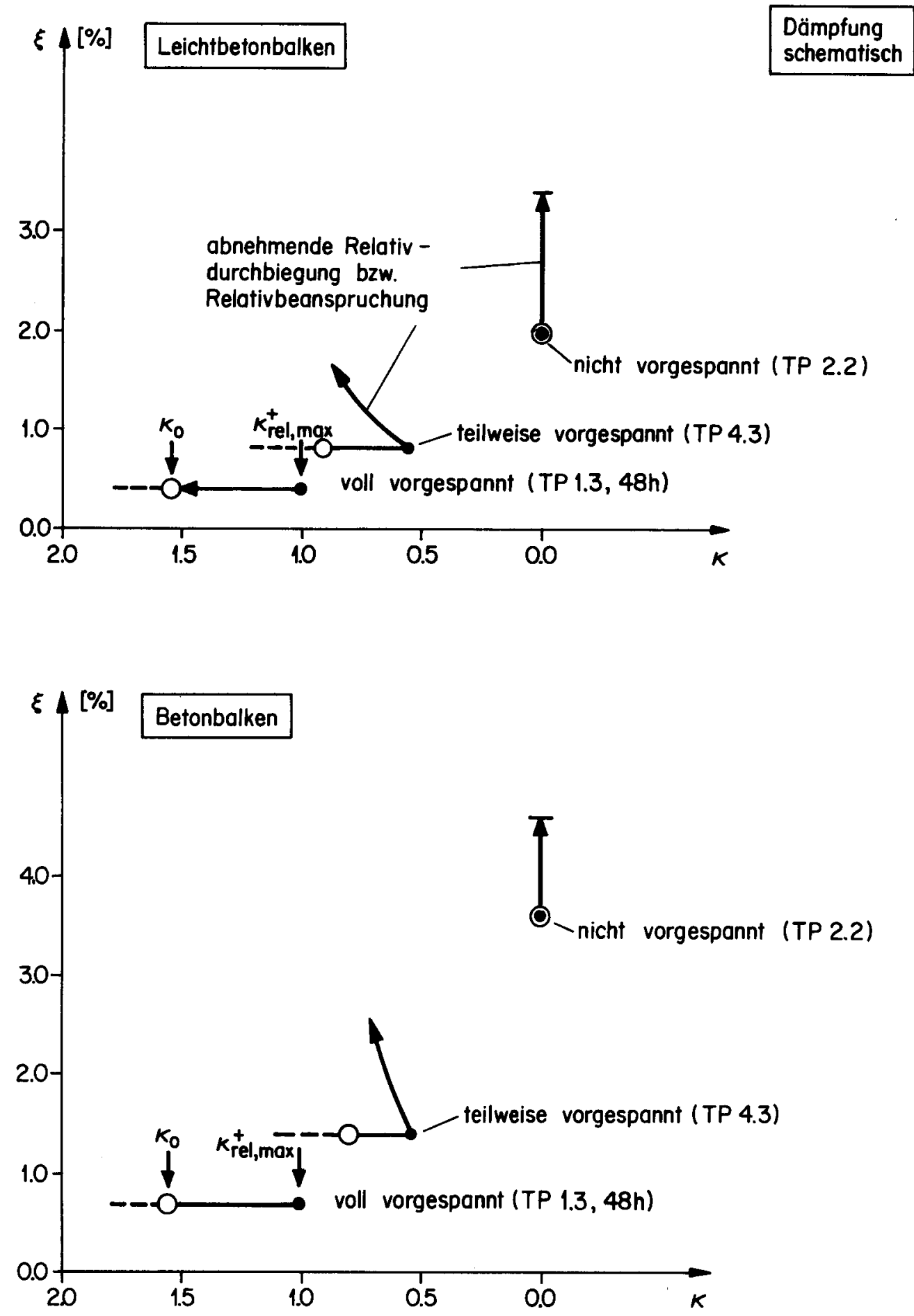

Bild 30c: Einfluss des Vorspanngrades auf die Dämpfung 

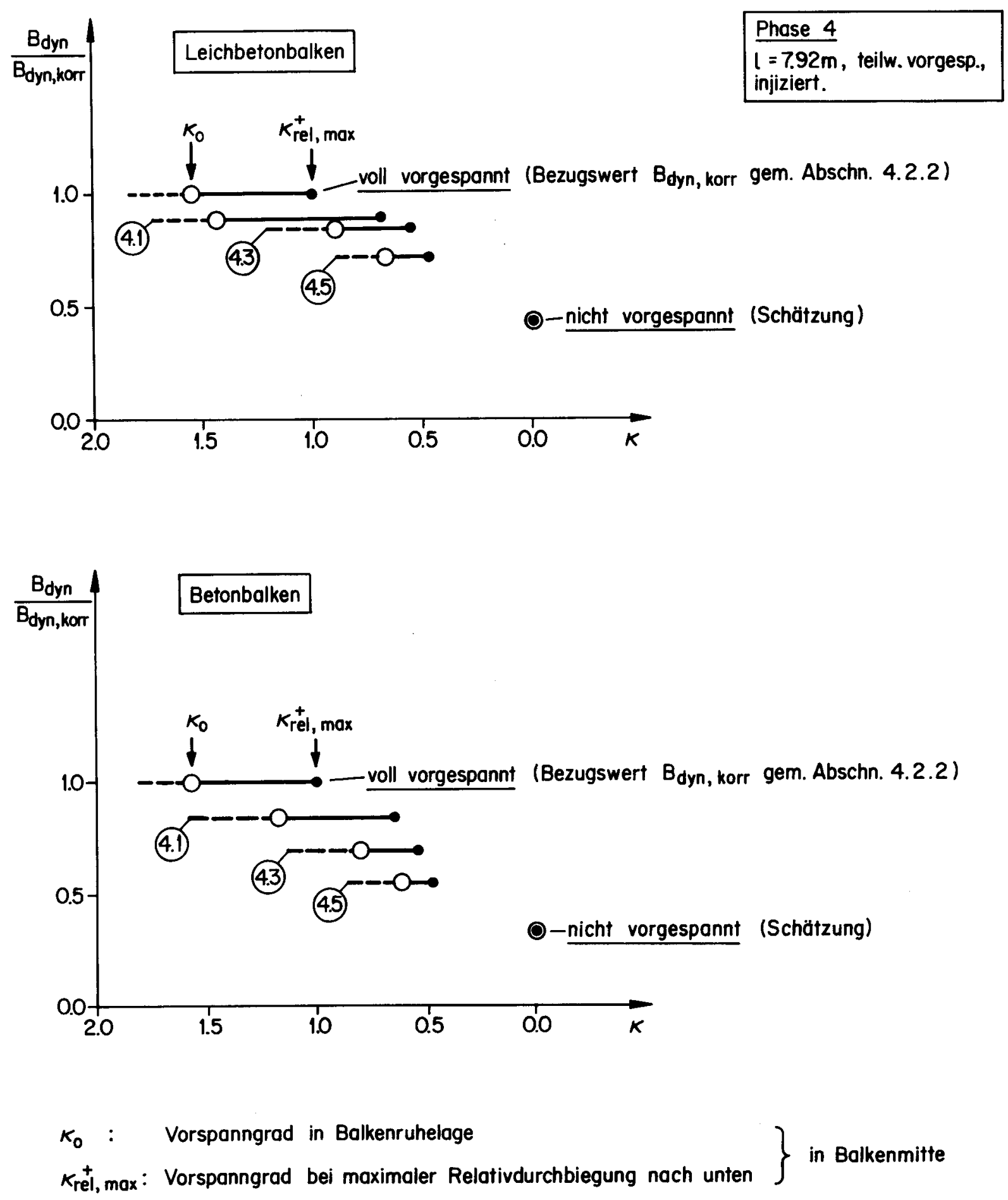

Bild 30a : Einfluss des Vorspanngrades auf die dynamische Biegesteifigkeit Phase 4 

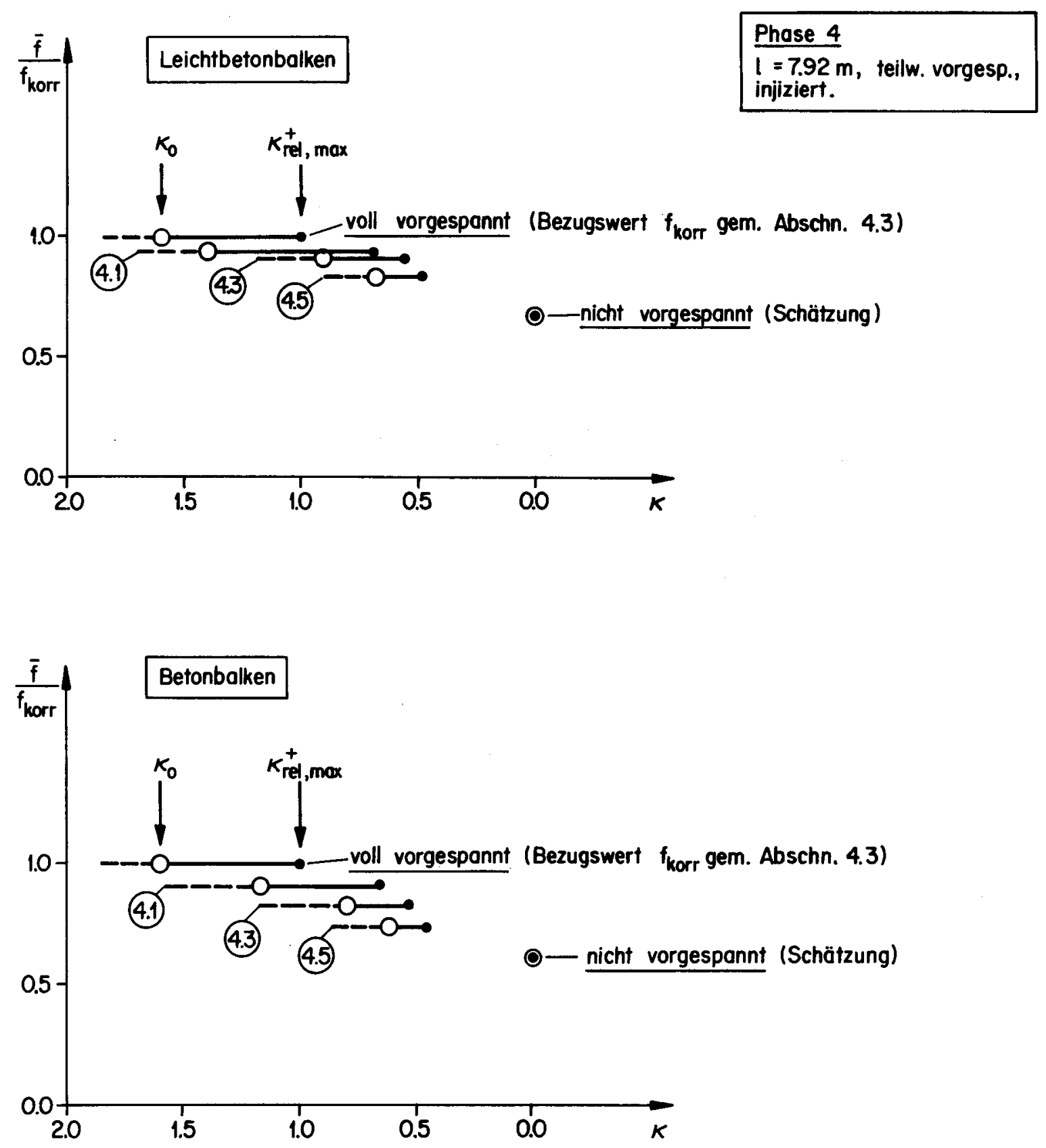

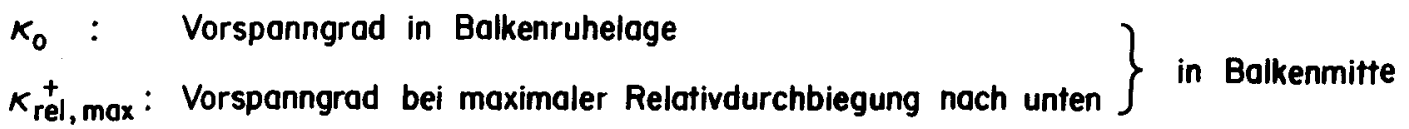

Bild 30b: Einfluss des Vorspanngrades auf die Eigenfrequenz, Phase 4 


\begin{tabular}{|c|c|c|}
\hline Variierter Parameter & \multicolumn{2}{|l|}{ Normalspannung (Variation der zentrischen Vorspannkraft) } \\
\hline Teilphasen & \multicolumn{2}{|l|}{$\begin{array}{l}1.1 \text { zentrische Vorspannkraft klein } \\
1.2 \text { zentrische Vorspannkraft mittel } \\
1.3 \text { zentrische Vorspannkraft gross }\end{array}$} \\
\hline Vorspannung & \multicolumn{2}{|c|}{$\begin{array}{l}\text { Zentrische Vorspannkraft mit sechs geraden Spannstäben ohne Verbund. } \\
\text { Volle Vorspannung in Balkenruhelage und bei maximalen Relativdurchbiegungen. } \\
\text { Gekrümmtes Spannglied nicht eingeführt. }\end{array}$} \\
\hline Belastung & \multicolumn{2}{|l|}{ Nur Eigengewicht, keine Zusatzmassen. } \\
\hline Risszustand & \multicolumn{2}{|l|}{ Keine Risse } \\
\hline Zweck & $\begin{array}{l}\text { 1. Einfluss der Grösse der zentrischen Vorspannkraft } \\
\text { auf Eigenfrequenz und Dämpfung des ungerissenen } \\
\text { Querschnittes. } \\
\text { 2. Einfluss des Versuchszeitpunktes nach Veränderung } \\
\text { der Vorspannkraft auf Eigenfrequenz und Dämpfung } \\
\text { des ungerissenen Querschnittes. }\end{array}$ & $\begin{array}{l}\text { Vergleich: } \\
\left.(1.1) \rightarrow\left(t_{1}\right) \rightarrow 1.3\right)\left(t_{2}\right)\end{array}$ \\
\hline Versuche & \multicolumn{2}{|c|}{$\begin{array}{l}\text { Pro Teilphase drei Ausschwingversuche jeweils } 1 \text { Stunde nach Veränderung der } \\
\text { Vorspannkraft. } \\
\text { Für die Teilphase } 1.3 \text { weitere Ausschwingversuche nach } 2,4,8,24,48 \text { Stunden } \\
\text { Keine statischen Belastungsversuche }\end{array}$} \\
\hline
\end{tabular}

Tabelle 1a: Kurzbeschrieb der Phase 1 (vg1. Bild 6a) 


\begin{tabular}{|c|c|c|}
\hline \multicolumn{3}{|c|}{ 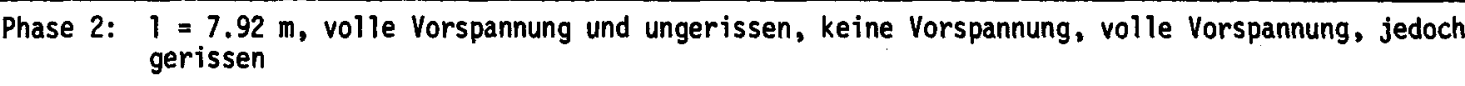 } \\
\hline Variierter Parameter & \multicolumn{2}{|l|}{ Risszustand (Variation durch Vorspannung) } \\
\hline Teilphasen & \multicolumn{2}{|c|}{$\begin{array}{l}\text { 2.1 Keine Risse. Volle Vorspannung in Balkenruhelage sowie bei maximalen } \\
\text { Relativdurchbiegungen } \\
2.2 \text { Volle Rissbildung, keine Vorspannung } \\
2.3 \text { Wie Teilphase } 2.1 \text {, jedoch mit überdrückten Rissen } \\
\text { 2.4 Wie Teilphase } 2.3 \text {, jedoch mit gekrümmtem Spannglied anstelle der zwei } \\
\text { exzentrischen geraden Spannstäbe }\end{array}$} \\
\hline Vorspannung & \multicolumn{2}{|c|}{$\begin{array}{l}\text { Exzentrische Vorspannkraft mit vier geraden Spannstäben ohne Verbund in } \\
\text { den Teilphasen } 2.1 \text { und } 2.3 \\
\text { Keine Vorspannung in der Teilphase } 2.2 \\
\text { Exzentrische Vorspannkraft mit gekriumntem Spannglied (nicht injiziert) mit } \\
\text { zwei zentrischen geraden Spannstäben ohne Verbund in der Teilphase } 2.4\end{array}$} \\
\hline Belastung & \multicolumn{2}{|l|}{ Nur Eigengewicht, keine Zusatzmassen } \\
\hline Zweck & $\begin{array}{l}\text { 1. Einfluss der Eigenfrequenz auf das Dämpfungsverhalten } \\
\text { des ungerissenen Querschnittes. } \\
\text { 2. Einfluss von überdrückten Rissen auf die Eigenfrequenz } \\
\text { die Dämpfung und die Steifigkeit. } \\
\text { 3. Ejnfluss eines gekrümmten, nichtinjizierten Spann- } \\
\text { gliedes auf die Eigenfrequenz, die Dämpfung und die } \\
\text { Steifigkeit. } \\
\text { 4. Bezugswerte zur 1. Versuchsserie an schlaff bewehrten } \\
\text { Balken [1]. } \\
\text { 5. Erzeugung des abgeschlossenen Risszustandes, um in den } \\
\text { Phasen } 3 \text { und } 4 \text { den Einfluss der Rissentstehung zu } \\
\text { eliminieren. }\end{array}$ & $\begin{array}{l}(2.3) \leftrightarrow 2.1 \\
2.4 \leftrightarrow 2.3 \\
2.2 \leftrightarrow[1]\end{array}$ \\
\hline Versuche & $\begin{array}{l}\text { Pro Teilphase drei Ausschwingversuche jeweils eine Stunde } \\
\text { der Vorspannkraft. } \\
\text { Pro Teilphase je ein statischer Belastungsversuch vor und } \\
\text { Ausschwingversuchen (Bestimnung der Biegesteifigkeit). }\end{array}$ & $\begin{array}{l}\text { nach Veränderung } \\
\text { nach den drei }\end{array}$ \\
\hline
\end{tabular}

Tabelle 1b: Kurzbeschrieb der Phase 2 (vgl. Bild 6b) 


\begin{tabular}{|c|c|c|}
\hline Variierter Parameter & \multicolumn{2}{|c|}{ Vorspanngrad (Variation durch Zusatzmassen) vgl. Tabelle 3} \\
\hline Teilphasen & \multicolumn{2}{|c|}{ 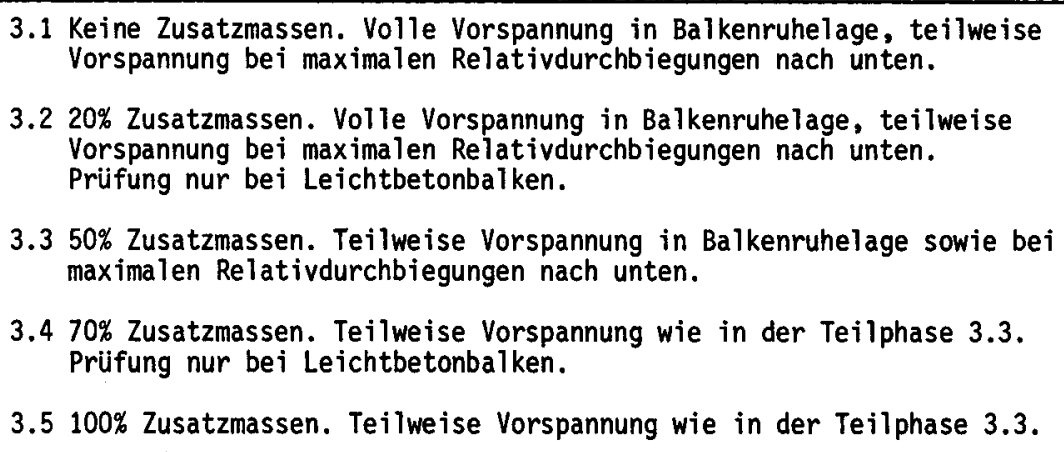 } \\
\hline Vorspannung & \multicolumn{2}{|c|}{$\begin{array}{l}\text { Exzentrische Vorspannkraft mit gekrümmtem nichtinjiziertem Spannglied. } \\
\text { Gerade Spannstangen nicht eingefüht. }\end{array}$} \\
\hline Risszustand & \multicolumn{2}{|l|}{ Vollständig gerissen } \\
\hline Zweck & $\begin{array}{l}\text { 1. Einfluss des Vorspanngrades auf die } \\
\text { Dämpfung, die Eigenfrequenz und die } \\
\text { Steifigkeit } \\
\text { 2. Einfluss der Betonart bei ca. gleicher Masse } \\
\text { und/oder ca. gleichem Vorspanngrad auf die } \\
\text { Eigenfrequenz, die Dämpfung und die Steifig- } \\
\text { keit }\end{array}$ & $\begin{array}{l}\text { Vergleich: } \\
\text { (2.4) } \leftrightarrow \text { (3.3.5 } \\
\text { (BB) B }\end{array}$ \\
\hline Versuche & \multicolumn{2}{|l|}{ Wie Phase 2} \\
\hline
\end{tabular}

Tabelle 1c: Kurzbeschrieb der Phase 3 (vgl. Bild 6c) 


\begin{tabular}{|c|c|c|}
\hline Variierter Parameter & \multicolumn{2}{|c|}{ Vorspanngrad (Variation durch Zusatzmassen) vgl. Tabelle 3} \\
\hline Teilphasen & \multicolumn{2}{|c|}{$\begin{array}{l}\text { 4.1 Keine Zusatzmassen. Volle Vorspannung in Balkenruhelage, } \\
\text { teilweise Vorspannung bei maximalen Relativdurchbiegungen nach unten. } \\
4.2 \text { } 20 \% \text { Zusatzmassen. Volle Vorspannung in Balkenruhelage, } \\
\text { teilweise Vorspannung bei maximalen Relativdurchbiegungen nach unten. } \\
\text { Prüfung nur bei Leichtbetonbalken. } \\
4.350 \% \text { Zusatzmassen. Teilweise Vorspannung in Balkenruhelage sowie bei } \\
\text { maximalen Relativdurchbiegungen nach unten. } \\
4.470 \% \text { Zusatzmassen. Teilweise Vorspannung wie in der Teilphase } 4.3 \text {. } \\
\text { Prüfung nur bei Leichtbetonbalken. } \\
4.5 \text { 100\% Zusatzmassen. Teilweise Vorspannung wie in der Teilphase } 4.3 \text {. }\end{array}$} \\
\hline Vorspannung & \multicolumn{2}{|c|}{$\begin{array}{l}\text { Exzentrische Vorspannkraft mit gekrümmtem injiziertem Spannglied. } \\
\text { Gerade Spannstangen nicht eingeführt. }\end{array}$} \\
\hline Risszustand & \multicolumn{2}{|l|}{ Vollständig gerissen } \\
\hline Zweck & $\begin{array}{l}\text { 1. Einfluss des Vorspanngrades auf die Dämpfung, } \\
\text { die Eigenfrequenz und die Steifigkeit. } \\
\text { 2. Einfluss der Betonart bei ca. gleicher Masse } \\
\text { und/oder ca. gleichem Vorspanngrad auf die } \\
\text { Eigenfrequenz, die Dämpfung und die Steifig- } \\
\text { keit. } \\
\text { 3. Einfluss der Injektion auf die Dämpfung, die } \\
\text { Eigenfrequenz und die Steifigkeit. }\end{array}$ & Phase (3) $\rightarrow$ Phase (4) \\
\hline Versuche & \multicolumn{2}{|l|}{ Wie in der Phase 2} \\
\hline
\end{tabular}

Tabelle 1d: Kurzbeschrieb der Phase 4 (vgl. Bild 6c) 


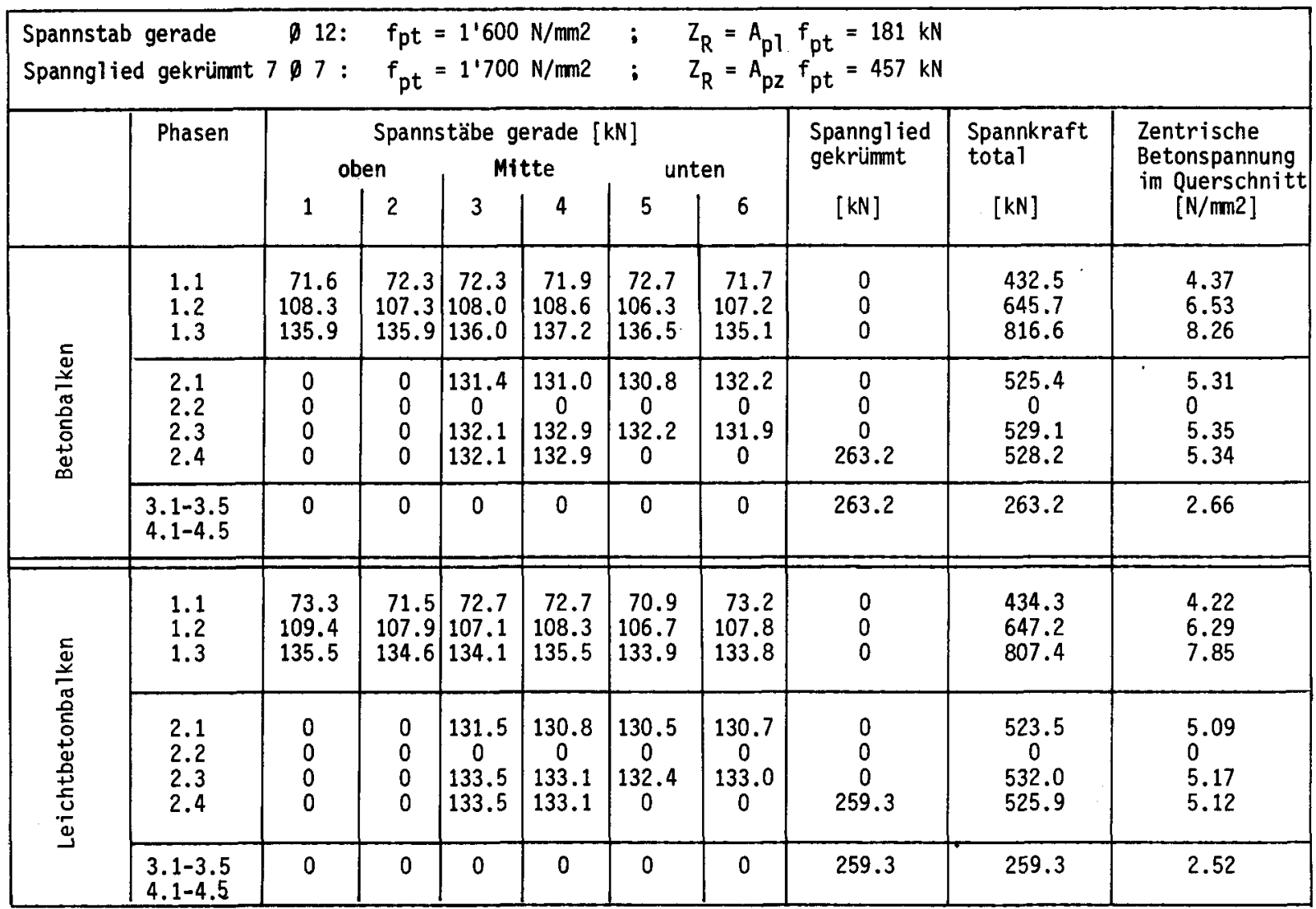

Tabelle 2: Vorspannkräfte (Messwerte)

\begin{tabular}{|c|c|c|c|c|}
\hline & Phase & $\begin{array}{c}\text { Vorspanngrad in } \\
\text { Balkenruhelage } \\
k_{0}\end{array}$ & $\begin{array}{c}\text { Vorspanngrad bei maximal } \\
\text { unten }(+) \text { bzw. nach oben } \\
\kappa_{\text {rel, max }}^{+}\end{array}$ & $\begin{array}{l}\text { durchbiegung nach } \\
\kappa_{r e 1, \max }^{-}\end{array}$ \\
\hline \multirow{2}{*}{ 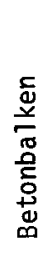 } & $\begin{array}{l}2.1 \\
2.2 \\
2.3 \\
2.4\end{array}$ & $\begin{array}{l}1.55 \\
0 \\
1.56 \\
1.62\end{array}$ & $\begin{array}{l}1.0 \\
0 \\
1.03 \\
1.06\end{array}$ & $\begin{array}{l}- \\
-\end{array}$ \\
\hline & $\begin{array}{l}3.1 ; 4.1 \\
3.3 ; 4.3 \\
3.5 ; 4.5\end{array}$ & $\begin{array}{l}1.17 \\
0.80 \\
0.61\end{array}$ & $\begin{array}{l}0.64 \\
0.54 \\
0.47\end{array}$ & $\begin{array}{l}1 . \overline{49} \\
0.85\end{array}$ \\
\hline \multirow{2}{*}{ 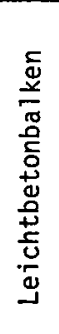 } & $\begin{array}{l}2.1 \\
2.2 \\
2.3 \\
2.4\end{array}$ & $\begin{array}{l}1.93 \\
0 \\
1.96 \\
2.03\end{array}$ & $\begin{array}{l}1.02 \\
0 \\
1.06 \\
1.10\end{array}$ & $\begin{array}{l}- \\
-\end{array}$ \\
\hline & $\begin{array}{l}3.1 ; 4.1 \\
3.2 ; 4.2 \\
3.3 ; 4.3 \\
3.4 ; 4.4 \\
3.5 ; 4.5\end{array}$ & $\begin{array}{l}1.45 \\
1.16 \\
0.91 \\
0.80 \\
0.67\end{array}$ & $\begin{array}{l}0.69 \\
0.63 \\
0.56 \\
0.53 \\
0.48\end{array}$ & $\begin{array}{l}- \\
2.39 \\
1.63 \\
1.10\end{array}$ \\
\hline
\end{tabular}

Tabelle 3: Rechnerische Vorspanngrade in Balkenmitte 


\begin{tabular}{|c|c|c|c|c|c|c|c|}
\hline \multicolumn{3}{|l|}{ Beton } & \multicolumn{3}{|l|}{ Leichtbeton } & \multicolumn{2}{|l|}{ Injektionsgut } \\
\hline Sand & $0-4 \mathrm{~mm}$ & $624 \mathrm{~kg} / \mathrm{m} 3$ & Sand & $0-4 \mathrm{~mm}$ & $575 \mathrm{~kg} / \mathrm{m} 3$ & Zement PC & $100 \mathrm{~kg}$ \\
\hline Sand & $4-8 \mathrm{~mm}$ & $195 \mathrm{~kg} / \mathrm{m} 3$ & hydraul. Kalk & & $50 \mathrm{~kg} / \mathrm{m} 3$ & Wasser & $38 \mathrm{~kg}$ \\
\hline Kies & $8-16 \mathrm{~mm}$ & $351 \mathrm{~kg} / \mathrm{m} 3$ & Leca-hade & $3-8 \mathrm{~mm}$ & $400 \mathrm{~kg} / \mathrm{m} 3$ & W/Z-Faktor & 0.38 \\
\hline Kies & $16-32 \mathrm{~mm}$ & $780 \mathrm{~kg} / \mathrm{m} 3$ & Leca-hade & $8-15 \mathrm{~mm}$ & $375 \mathrm{~kg} / \mathrm{m} 3$ & Zusatzmittel: & \\
\hline Zemen & & $300 \mathrm{~kg} / \mathrm{m} 3$ & Zement PC & & $350 \mathrm{~kg} / \mathrm{m} 3$ & Intracrete EH & $0.75 \mathrm{~kg}$ \\
\hline Wasse & & $150 \mathrm{~kg} / \mathrm{m} 3$ & Wasser & & $\sim 210 \mathrm{~kg} / \mathrm{m} 3$ & & \\
\hline$W / Z-F$ & & 0.5 & W/Z-Faktor & & $\sim 0.60$ & & \\
\hline Raumg & & $2^{\prime} 400 \mathrm{~kg} / \mathrm{m} 3$ & Raumgewicht $\gamma$ & & $1 ' 960 \mathrm{~kg} / \mathrm{m} 3$ & . & \\
\hline Konsi & & weichplastisch & Konsistenz & & ichplastisch & & \\
\hline
\end{tabular}

Tabelle 4: Zusarmensetzung von Beton, Leichtbeton und Injektionsgut

\begin{tabular}{|c|c|c|c|c|c|c|c|}
\hline \multicolumn{3}{|c|}{$\begin{aligned} \text { Priifkörper: Wiirfel } 0.15 \times 0.15 \times 0.15 \mathrm{~m} \\
$\[ \mathrm{zy} \text { linder } 0.15 \times 0.30 \mathrm{~m} \]$\end{aligned}$} & \multicolumn{2}{|c|}{$\begin{array}{l}\rightarrow \gamma, f_{C W} \\
+E, f_{C}\end{array}$} & \multirow[b]{2}{*}{$\stackrel{f_{C}}{[N / m m 2]}$} & \multirow[b]{2}{*}{$\begin{array}{l}E_{5-10} \\
{[\mathrm{~N} / \mathrm{mm} 2]}\end{array}$} & \multirow[b]{2}{*}{$\begin{array}{l}E_{5-20} \\
{[\mathrm{~N} / \mathrm{mm} 2]}\end{array}$} \\
\hline & $\begin{array}{l}\text { Anzahl } \\
\text { Proben }\end{array}$ & $\begin{array}{l}\text { Proben- } \\
\text { alter } \\
\text { Tage }\end{array}$ & $\begin{array}{c}\gamma \\
{[\mathrm{kN} / \mathrm{m} 3]}\end{array}$ & $\begin{array}{l}f_{C W} \\
{[N / \mathrm{mm} 2]}\end{array}$ & & & \\
\hline \multirow[t]{2}{*}{ Beton } & 4 & 29 & 23.7 & 42 & & & \\
\hline & 2 & 29 & & & 33 & $31^{\prime} 620$ & $28 \cdot 950$ \\
\hline \multirow[t]{2}{*}{ Leichtbeton } & 4 & 34 & 17.27 & 44 & & & \\
\hline & 2 & 34 & & & 39 & $18^{\prime} 950$ & $18^{\prime} 220$ \\
\hline \multicolumn{8}{|l|}{ Injektionsgut: } \\
\hline - Betonbalken & 4 & 21 & 17.21 & - & 24 & - & - \\
\hline - Leichtbetonbalken & 4 & 23 & 17.52 & - & 25 & - & - \\
\hline
\end{tabular}

Tabelle 5: Eigenschaften von Beton, Leichtbeton und Injektionsgut

\begin{tabular}{|c|c|c|c|c|c|}
\hline Stahlsorte & $\begin{array}{c}\text { A } \\
\text { [mm2] }\end{array}$ & $\begin{array}{c}f_{y} \\
{\left[N / \mathrm{mm}^{2}\right]}\end{array}$ & $\begin{array}{c}f_{t} \\
{[\mathrm{~N} / \mathrm{mm} 2]}\end{array}$ & $\begin{array}{c}E \\
{[N / m m 2]}\end{array}$ & $\lambda_{\mathrm{GI}}$ \\
\hline Staht $S 500 \emptyset 12 \mathrm{~mm}$ (TORIP) & 113 & 520 & 580 & $210^{\prime} 000$ & $13 \%$ \\
\hline Spannstah1 $12 \mathrm{~mm}$ (BBRV) & 113 & $1 ' 440$ & $1^{\prime} 600$ & $210^{\prime} 000$ & $2 \%$ \\
\hline Spannstah1 $\emptyset \quad 7 \mathrm{~mm}$ (BBRV) & 38.5 & $1 ' 530$ & $1 \cdot 700$ & $210^{\prime} 000$ & $2 \%$ \\
\hline
\end{tabular}

Tabelle 6: Eigenschaften der Stähle (garantierte Werte der Stahllieferanten) 


\begin{tabular}{|c|c|c|c|c|c|c|c|c|c|}
\hline & $\begin{array}{l}\text { Querschnitts- } \\
\text { Typ gemäss } \\
\text { Bild } 15\end{array}$ & $\begin{array}{l}A_{c} \\
{[\mathrm{~mm} 2]}\end{array}$ & $\begin{array}{c}A_{s}=A_{s}^{1} \\
{[\mathrm{~mm} 2]}\end{array}$ & $\begin{array}{c}A_{p 1} \\
{[\mathrm{~mm} 2]}\end{array}$ & $\begin{array}{c}A_{p 2} \\
{[\mathrm{~mm} 2]}\end{array}$ & $\begin{array}{c}A_{i} \\
{[\mathrm{~mm} 2]}\end{array}$ & $\begin{array}{c}y \\
{[\mathrm{~mm}]}\end{array}$ & $\begin{array}{l}\mathrm{I}_{i} \\
{[\mathrm{~mm} 4]}\end{array}$ & $\begin{array}{c}B \\
{[\mathrm{~N} / \mathrm{mm} 2]}\end{array}$ \\
\hline Beton & $\begin{array}{l}A \\
B \\
C \\
D \\
\text { E } \\
\text { F nichtinjiziert } \\
\text { F injiziert }\end{array}$ & $\begin{array}{l}96^{\prime}, 000 \\
92 ' 551 \\
92^{\prime} 551 \\
92^{\prime} 551 \\
92^{\prime} 551 \\
92^{\prime} 551 \\
93^{\prime} \cdot 300\end{array}$ & $\begin{array}{l}452 \\
452 \\
452 \\
452 \\
452 \\
452 \\
452\end{array}$ & $\begin{array}{c}0 \\
0 \\
679 \\
453 \\
227 \\
0 \\
0\end{array}$ & $\begin{array}{c}0 \\
0 \\
0 \\
0 \\
269 \\
269 \\
269\end{array}$ & $\begin{array}{r}96 \cdot 000 \\
98 \cdot 879 \\
101 \cdot 731 \\
100 \cdot 781 \\
160 ' 962 \\
100 \cdot 013 \\
101^{\prime} \cdot 511\end{array}$ & $\begin{array}{l}120 \\
119 \\
119 \\
120 \\
120 \\
120 \\
121\end{array}$ & $\begin{array}{l}460.8 \\
491.6 \\
498.5 \\
494.9 \\
495.5 \\
495.5 \\
506.2\end{array}$ & $\begin{array}{l}13.82 \\
14.75 \\
14.96 \\
14.85 \\
14.87 \\
14.87 \\
15.19\end{array}$ \\
\hline $\begin{array}{l}\text { Leicht- } \\
\text { beton }\end{array}$ & $\begin{array}{c}A \\
B \\
C \\
D \\
E \\
\text { F nichtinjiziert } \\
\text { F injiziert }\end{array}$ & $\begin{array}{l}96 \cdot 000 \\
92 ' 551 \\
92 \cdot 551 \\
92 \cdot 551 \\
92 \cdot 551 \\
92 \cdot 551 \\
93^{\prime} \cdot 300\end{array}$ & $\begin{array}{l}452 \\
452 \\
452 \\
452 \\
452 \\
452 \\
452\end{array}$ & $\begin{array}{c}0 \\
0 \\
679 \\
453 \\
227 \\
0 \\
0\end{array}$ & $\begin{array}{c}0 \\
0 \\
0 \\
0 \\
269 \\
269 \\
269\end{array}$ & $\begin{array}{r}96 ' 000 \\
102 ' 813 \\
107 ' 438 \\
105 ' 893 \\
106^{\prime} 184 \\
104^{\prime} \cdot 647 \\
106^{\prime} 614\end{array}$ & $\begin{array}{l}120 \\
119 \\
119 \\
120 \\
120 \\
120 \\
121\end{array}$ & $\begin{array}{l}460.8 \\
520.7 \\
531.8 \\
526.1 \\
527.1 \\
527.1 \\
541.7\end{array}$ & $\begin{array}{r}8.52 \\
9.63 \\
9.84 \\
9.73 \\
9.75 \\
9.75 \\
10.02\end{array}$ \\
\hline
\end{tabular}

$y=$ Abstand der Schwerachse zum oberen Rand

Tabelle 7: Rechnerische Querschnittswerte im ungerissenen Zustand für reine Biegung (Verbundkoeffizient $\bar{k}=1.0$ )

\begin{tabular}{|c|c|c|c|c|c|c|}
\hline & $\begin{array}{l}\text { Querschnitts- } \\
\text { Typ gemäss } \\
\text { Bild } 9\end{array}$ & $\begin{array}{l}A_{s}=A_{s}^{\prime} \\
{[\mathrm{mm} 2]}\end{array}$ & $\begin{array}{l}A_{p 2} \\
{[\mathrm{~mm} 2]}\end{array}$ & $\begin{array}{c}x \\
{[\mathrm{~mm}]}\end{array}$ & $\begin{array}{c}I_{i} \\
{[\mathrm{~mm} 4]} \\
\times 10^{6}\end{array}$ & $\begin{array}{c}\text { B } \\
{[\mathrm{N} / \mathrm{mm} 2]} \\
\times 10^{12}\end{array}$ \\
\hline Beton & $\begin{array}{l}\text { B } \\
\text { F nichtinjiziert } \\
\text { F injiziert }\end{array}$ & $\begin{array}{l}452 \\
452 \\
452\end{array}$ & $\begin{array}{l}- \\
269 \\
269\end{array}$ & $\begin{array}{l}48 \\
54 \\
57\end{array}$ & $\begin{array}{r}96.4 \\
117.8 \\
131.7\end{array}$ & $\begin{array}{l}2.83 \\
3.53 \\
3.95\end{array}$ \\
\hline $\begin{array}{l}\text { Leicht- } \\
\text { beton }\end{array}$ & $\begin{array}{l}B \\
\text { F nichtinjiziert } \\
F \text { injiziert }\end{array}$ & $\begin{array}{l}452 \\
452 \\
452\end{array}$ & $\begin{array}{c}- \\
269 \\
269\end{array}$ & $\begin{array}{l}57 \\
64 \\
68\end{array}$ & $\begin{array}{l}141.3 \\
172.9 \\
197.7\end{array}$ & $\begin{array}{l}2.61 \\
3.20 \\
3.66\end{array}$ \\
\hline
\end{tabular}

$x=$ Neutralachsenabstand zum oberen Rand

Tabelle 8: Rechnerische Querschnittswerte im gerissenen Zustand für reine Biegung (Verbundkoeffizient $\bar{k}=1.0$ )

\begin{tabular}{|l|c|c|}
\hline & $\begin{array}{c}\text { Betonbalken } \\
{\left[\mathrm{kN} / \mathrm{m}^{\prime}\right]}\end{array}$ & $\begin{array}{c}\text { Leichtbetonbalken } \\
{\left[\mathrm{kN} / \mathrm{m}^{\prime}\right]}\end{array}$ \\
\hline Balkeneigengewicht $\mathrm{g}$ & 2.34 & 1.74 \\
\hline Balkeneigengewicht $g+$ Gewicht q der Zusatzmassen: & & \\
Teilphasen 3.2 und 4.2: $\Delta \mathrm{M}=20 \%$ & - & 2.36 \\
Teilphasen 3.3 und 4.3: $=50 \%$ & 3.82 & 3.22 \\
Teilphasen 3.4 und 4.4: $=70 \%$ & - & 3.79 \\
Teilphasen 3.5 und 4.5: $=100 \%$ & 5.25 & 4.65 \\
\hline Schwinger in Balkenmitte $G_{S}[\mathrm{kN}]$ & & 3.1 \\
Ankerplatten an Balkenenden $G_{A}[\mathrm{kN}]$ & & je \\
\hline
\end{tabular}

Tabelle 9: Gewichte 


\begin{tabular}{|c|c|c|c|c|c|c|c|}
\hline & \multirow[t]{2}{*}{ Teilphasen } & \multirow[b]{2}{*}{$\begin{array}{c}M_{0} \\
{[k N m]}\end{array}$} & \multicolumn{2}{|c|}{ Balkenmitte } & \multicolumn{3}{|c|}{ über dem Auflager } \\
\hline & & & $\underset{[\mathrm{kNm}]}{M_{p}}$ & $\underset{\text { op }}{M_{\mathrm{kNm}]}}$ & $\begin{array}{c}M_{0} \\
{[\mathrm{kNm}]}\end{array}$ & $\stackrel{M p}{p}_{[\mathrm{kNm}]}$ & $\underset{[\mathrm{kNm}]}{\mathrm{M}_{\mathrm{op}}}$ \\
\hline \multirow{3}{*}{$\begin{array}{l}\text { Beton- } \\
\text { balken }\end{array}$} & $1.1-1.3$ & 7.7 & 0 & 7.7 & -3.7 & 0 & -3.7 \\
\hline & $\begin{array}{l}2.1 \\
2.2 \\
2.3 \\
2.4\end{array}$ & $\begin{array}{l}24.4 \\
24.4 \\
24.4 \\
24.4\end{array}$ & $\begin{array}{l}-15.8 \\
0 \\
-15.9 \\
-17.6\end{array}$ & $\begin{array}{r}8.6 \\
24.4 \\
8.5 \\
6.8\end{array}$ & $\begin{array}{l}-0.12 \\
-0.12 \\
-0.12 \\
-0.12\end{array}$ & $\begin{array}{c}-15.8 \\
0 \\
-15.9 \\
0\end{array}$ & $\begin{array}{l}-15.9 \\
-0.12 \\
-16.0 \\
-0.12\end{array}$ \\
\hline & $\begin{array}{ll}3.1, & 4.1 \\
3.3, & 4.3 \\
3.5, & 4.5\end{array}$ & $\begin{array}{l}24.4 \\
35.9 \\
47.1\end{array}$ & $\begin{array}{l}-17.6 \\
-17.6 \\
-17.6\end{array}$ & $\begin{array}{r}6.8 \\
18.3 \\
29.5\end{array}$ & $\begin{array}{l}-0.12 \\
-0.16 \\
-0.20\end{array}$ & $\begin{array}{l}0 \\
0 \\
0\end{array}$ & $\begin{array}{l}-0.12 \\
-0.16 \\
-0.20\end{array}$ \\
\hline \multirow{3}{*}{$\begin{array}{l}\text { Leicht- } \\
\text { beton- } \\
\text { balken }\end{array}$} & $1.1-1.3$ & 6.4 & 0 & 6.4 & -2.8 & 0 & -2.8 \\
\hline & $\begin{array}{l}2.1 \\
2.2 \\
2.3 \\
2.4\end{array}$ & $\begin{array}{l}19.7 \\
19.7 \\
19.7 \\
19.7\end{array}$ & $\begin{array}{c}-15.7 \\
0 \\
-15.9 \\
-17.4\end{array}$ & $\begin{array}{r}4.0 \\
19.7 \\
3.8 \\
2.3\end{array}$ & $\begin{array}{l}-0.10 \\
-0.10 \\
-0.10 \\
-0.10\end{array}$ & $\begin{array}{c}-15.7 \\
0 \\
-15.9 \\
0\end{array}$ & $\begin{array}{l}-15.8 \\
-0.10 \\
-16.0 \\
-0.10\end{array}$ \\
\hline & $\begin{array}{ll}3.1,4.1 \\
3.2, & 4.2 \\
3.3, & 4.3 \\
3.4, & 4.4 \\
3.5, & 4.5\end{array}$ & $\begin{array}{l}19.7 \\
24.5 \\
31.3 \\
35.7 \\
42.4\end{array}$ & $\begin{array}{l}-17.4 \\
-17.4 \\
-17.4 \\
-17.4 \\
-17.4\end{array}$ & $\begin{array}{r}2.3 \\
7.1 \\
13.9 \\
18.3 \\
25.0\end{array}$ & $\begin{array}{l}-0.10 \\
-0.12 \\
-0.14 \\
-0.16 \\
-0.18\end{array}$ & $\begin{array}{l}0 \\
0 \\
0 \\
0 \\
0\end{array}$ & $\begin{array}{l}-0.10 \\
-0.12 \\
-0.14 \\
-0.16 \\
-0.18\end{array}$ \\
\hline \multicolumn{8}{|c|}{$\begin{array}{l}M_{0}=\text { Biegemoment in Balkenruhelage infolge Balkeneigengewichtes, Zusatzmassen, Schwinger } \\
\text { und Ankerplatten } \\
M_{n}=\text { Moment infolge Vorspannung }\end{array}$} \\
\hline
\end{tabular}

Tabelle 10: Rechnerische Biegemomente in Balkenruhelage

\begin{tabular}{|c|c|c|c|c|}
\hline \multirow[t]{2}{*}{ Teilphasen } & \multicolumn{2}{|c|}{ Betonbalken } & \multicolumn{2}{|c|}{ Leichtbetonbalken } \\
\hline & $\begin{array}{l}\text { Balkenmitte } \\
\text { [N.sec2] }\end{array}$ & $\begin{array}{l}\text { Auflager } \\
\text { [N.sec2] }\end{array}$ & $\begin{array}{l}\text { Balkenmitte } \\
\text { [N-sec2] }\end{array}$ & $\begin{array}{l}\text { Auflager } \\
{[N \cdot \text { sec2] }}\end{array}$ \\
\hline $1.1-1.3$ & $53.0 \cdot 10^{3}$ & $13.1 \cdot 10^{3}$ & $43.7 \cdot 10^{3}$ & $10.1 \cdot 10^{3}$ \\
\hline $2.1-2.4$ & $84.2 \cdot 10^{3}$ & 0 & $68.9 \cdot 10^{3}$ & 0 \\
\hline $\begin{array}{l}3.1,4.1 \\
3.2,4.2 \\
3.3,4.3 \\
3.4,4.4 \\
3.5,4.5\end{array}$ & $\begin{array}{c}84.2 \cdot 10^{3} \\
- \\
121.8 \cdot 10^{3} \\
- \\
158.1 \cdot 10^{3}\end{array}$ & $\begin{array}{l}0 \\
\overline{0} \\
\overline{0} \\
0\end{array}$ & $\begin{array}{r}68.9 \cdot 10^{3} \\
84.7 \cdot 10^{3} \\
106.5 \cdot 10^{3} \\
121.0 \cdot 10^{3} \\
142.8 \cdot 10^{3}\end{array}$ & $\begin{array}{l}0 \\
0 \\
0 \\
0 \\
0\end{array}$ \\
\hline \multicolumn{5}{|c|}{ Moment $=$ Tabellenwert $\times$ Frequen $z^{2} \times$ Relativdurchbiegung in Balkenmitte } \\
\hline \multicolumn{5}{|c|}{$\begin{array}{l}\text { Annahmen: Biegesteifigkeit unabhängig von der Relativdurchbiegung und konstant } 1 \text { ängs } \\
\text { der Balkenachse }\end{array}$} \\
\hline
\end{tabular}

Tabelle 11: Rechnerische Biegemomente für eine dynamische Relativdurchbiegung in Balkenmitte von $w_{\text {rel }}=1 \mathrm{~mm}$ bei einer Eigenfrequenz $f=1 \mathrm{~Hz}$ 


\begin{tabular}{|c|c|c|}
\hline Teilphasen & $\begin{array}{l}\text { Betonbalken } \\
w_{\mathrm{re}}^{+}, \max \\
{[\mathrm{mm}]}\end{array}$ & $\begin{array}{c}\text { Leichtbetonbalken } \\
w_{\text {rel }}^{+} \text {, max } \\
{[\mathrm{mm}]}\end{array}$ \\
\hline $\begin{array}{l}1.1 \\
1.2 \\
1.3\end{array}$ & $\begin{array}{l}+1.0 \\
+2.0 \\
+3.0\end{array}$ & $\begin{array}{l}+2.0 \\
+3.0 \\
+4.0\end{array}$ \\
\hline $\begin{array}{l}2.1 \\
2.2 \\
2.3 \\
2.4\end{array}$ & $\begin{array}{r}+5.0 \\
+10.0 \\
+\quad 5.0 \\
+\quad 5.0\end{array}$ & $\begin{array}{l}+10.0 \\
+20.0 \\
+10.0 \\
+10.0\end{array}$ \\
\hline $\begin{array}{ll}3.1, & 4.1 \\
3.2, & 4.2 \\
3.3, & 4.3 \\
3.4, & 4.4 \\
3.5, & 4.5\end{array}$ & $\begin{array}{l}+15.0 \\
+\overline{15.0} \\
+\overline{-} \\
+15.0\end{array}$ & $\begin{array}{l}+20.0 \\
+20.0 \\
+20.0 \\
+20.0 \\
+20.0\end{array}$ \\
\hline
\end{tabular}

Tabelle 12: Für Versuchssteuerung gewählte maximale Relativdurchbiegungen nach unten $w_{r e l, m a x}^{+}$in Balkenmitte

\begin{tabular}{|c|c|c|c|c|c|c|c|c|c|}
\hline \multicolumn{2}{|c|}{$\begin{array}{l}\text { Relativdurchbiegung } \\
\text { nach unten wrel } \\
=\text { = Steuergrösse }\end{array}$} & $\begin{array}{l}+1.0 \\
{[\mathrm{~mm}]}\end{array}$ & $\begin{array}{r}+2.0 \\
{[\mathrm{~mm}]}\end{array}$ & $\begin{array}{l}+3.0 \\
{[\mathrm{~mm}]}\end{array}$ & $\begin{array}{l}+4.0 \\
{[\mathrm{~mm}]}\end{array}$ & $\begin{array}{l}+5.0 \\
{[\mathrm{~mm}]}\end{array}$ & $\begin{array}{l}+10.0 \\
{[\mathrm{~mm}]}\end{array}$ & $\begin{array}{r}+15.0 \\
{[\mathrm{~mm}]}\end{array}$ & $\begin{array}{c}+20.0 \\
{[\mathrm{~mm}]}\end{array}$ \\
\hline \multicolumn{10}{|c|}{$\begin{array}{l}\text { Re lativdurchbiegung } \\
\text { nach oben wrel } \\
=\text { gemessene Grösse }\end{array}$} \\
\hline \multirow{4}{*}{$\begin{array}{l}\text { Beton- } \\
\text { balken }\end{array}$} & $\begin{array}{c}\text { Teilphasen } \\
1.1 \\
1.2 \\
1.3 \\
\end{array}$ & -0.99 & -1.97 & -2.96 & & & & & \\
\hline & $\begin{array}{l}2.1 \\
2.2 \\
2.3 \\
2.4 \\
\end{array}$ & & & $\begin{array}{l}-2.91 \\
-2.93 \\
-2.94 \\
\end{array}$ & & $\begin{array}{l}-4.88 \\
-5.20 \\
-4.86 \\
-4.88 \\
\end{array}$ & -10.66 & & \\
\hline & $\begin{array}{l}3.1 \\
3.3 \\
3.5 \\
\end{array}$ & & & & & $\begin{array}{l}-4.81 \\
-4.82 \\
-4.81 \\
\end{array}$ & $\begin{array}{l}-9.26 \\
-\quad 9.13 \\
-\quad 9.38 \\
\end{array}$ & $\begin{array}{l}-13.62 \\
-12.80 \\
-13.88 \\
\end{array}$ & \\
\hline & $\begin{array}{l}4.1 \\
4.3 \\
4.5 \\
\end{array}$ & & & & & $\begin{array}{l}-5.02 \\
-4.98 \\
-4.94 \\
\end{array}$ & $\begin{array}{l}-9.88 \\
-\quad 9.38 \\
-\quad 9.65 \\
\end{array}$ & $\begin{array}{l}-14.50 \\
-13.75 \\
-14.15 \\
\end{array}$ & \\
\hline \multirow{4}{*}{$\begin{array}{l}\text { Leicht- } \\
\text { beton- } \\
\text { balken }\end{array}$} & $\begin{array}{l}1.1 \\
1.2 \\
1.3 \\
\end{array}$ & & -2.00 & -3.00 & -3.97 & & & & \\
\hline & $\begin{array}{l}2.1 \\
2.2 \\
2.3 \\
2.4 \\
\end{array}$ & & & & & $\begin{array}{l}-5.02 \\
-5.06 \\
-5.10 \\
\end{array}$ & $\begin{array}{l}-10.00 \\
-10.30 \\
-10.05 \\
-10.13 \\
\end{array}$ & -15.45 & -20.80 \\
\hline & $\begin{array}{l}3.1 \\
3.2 \\
3.3 \\
3.4 \\
3.5 \\
\end{array}$ & & & & & . & $\begin{array}{rr}- & 10.13 \\
-\quad 9.80 \\
-\quad 9.25 \\
-\quad 9.25 \\
-\quad 9.13 \\
\end{array}$ & $\begin{array}{l}-15.15 \\
-14.50 \\
=13.45 \\
=13.40 \\
-13.28 \\
\end{array}$ & $\begin{array}{l}-20.05 \\
-19.11 \\
-17.63 \\
-17.40 \\
-17.23 \\
\end{array}$ \\
\hline & $\begin{array}{l}4.1 \\
4.2 \\
4.3 \\
4.4 \\
4.5\end{array}$ & & & & & & $\begin{array}{lr}- & 10.25 \\
-\quad 9.78 \\
-\quad 9.55 \\
-\quad 9.28 \\
-\quad 9.52 \\
\end{array}$ & $\begin{array}{l}-15.20 \\
-14.75 \\
=14.25 \\
=13.57 \\
-14.02 \\
\end{array}$ & $\begin{array}{l}-20.25 \\
-19.75 \\
-18.96 \\
-18.00 \\
-18.48 \\
\end{array}$ \\
\hline
\end{tabular}

Tabelle 13: Relativdurchbiegung nach unten $w_{r e]}^{+}(=$gewählt) und zugehörige Relativdurchbiegung nach oben $w_{\text {rel }}^{-}(=$gemessen) in Balkenmitte beim Ausschwingversuch 


\begin{tabular}{|c|c|c|c|c|c|c|c|c|}
\hline & $\begin{array}{l}\text { Teil- } \\
\text { phase }\end{array}$ & $\begin{array}{l}\text { Dehnuns } \\
\text { Beton } \\
\end{array}$ & $\begin{array}{l}\text { ssrahmen } \\
\% 0]\end{array}$ & $\begin{array}{l}\text { Balkenmitte } \\
\text { schlaffer } \\
\end{array}$ & ahl $\varepsilon_{s}[\% 0]$ & $\begin{array}{l}\text { Rechn. } \\
\text { Spanni } \\
\text { Beton }\end{array}$ & $\begin{array}{l}\text { ungen i } \\
\text { gemäss } \\
\text { o] }\end{array}$ & $\begin{array}{l}\text { mitte aus } \\
\text { affer } S \operatorname{tah} 1 \varepsilon_{s}\end{array}$ \\
\hline \multirow{5}{*}{ 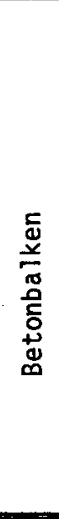 } & & unten & oben & unten & & unten & oben & unten \\
\hline & $\begin{array}{l}1.1 \\
1.2 \\
1.3 \\
\end{array}$ & $\begin{array}{l}0.090 \\
0.186 \\
0.278 \\
\end{array}$ & $\begin{array}{l}0.096 \\
0.192 \\
0.284 \\
\end{array}$ & $\begin{array}{l}0.068 \\
0.143 \\
0.200 \\
\end{array}$ & & $\begin{array}{l}0.10 \\
0.206 \\
0.308 \\
\end{array}$ & $\begin{array}{l}0.10 \\
0.206 \\
0.308 \\
\end{array}$ & $\begin{array}{l}0.070 \\
0.148 \\
0.220 \\
\end{array}$ \\
\hline & $\begin{array}{l}2.1 \\
2.2 \\
2.3 \\
2.4 \\
\end{array}$ & $\begin{array}{l}0.210 \\
- \\
0.207 \\
0.195 \\
\end{array}$ & $\begin{array}{l}0.204 \\
0.249 \\
0.198 \\
0.195 \\
\end{array}$ & $\begin{array}{l}0.156 \\
0.608 \\
0.156 \\
0.158 \\
\end{array}$ & & $\begin{array}{l}0.204 \\
- \\
0.196 \\
0.195 \\
\end{array}$ & $\begin{array}{l}0.204 \\
0.250 \\
0.196 \\
0.195 \\
\end{array}$ & $\begin{array}{l}0.146 \\
0.848 \\
0.140 \\
0.142 \\
\end{array}$ \\
\hline & $\begin{array}{l}3.1 \\
3.3 \\
3.5 \\
\end{array}$ & - & $\begin{array}{l}0.54 \\
0.46 \\
0.41 \\
\end{array}$ & $\begin{array}{l}0.56 \\
0.68 \\
0.70 \\
\end{array}$ & & - & - & $\begin{array}{l}- \\
-\end{array}$ \\
\hline & $\begin{array}{l}4.1 \\
4.3 \\
4.5\end{array}$ & $\begin{array}{l}- \\
-\end{array}$ & $\begin{array}{l}0.58 \\
0.47 \\
0.40 \\
\end{array}$ & $\begin{array}{l}0.58 \\
0.63 \\
0.66 \\
\end{array}$ & & - & $\begin{array}{l}0.336 \\
0.317 \\
0.304 \\
\end{array}$ & $\begin{array}{l}0.348 \\
0.438 \\
0.514 \\
\end{array}$ \\
\hline \multirow{4}{*}{ 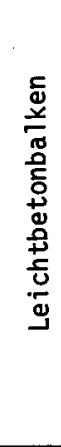 } & $\begin{array}{l}1.1 \\
1.2 \\
1.3 \\
\end{array}$ & $\begin{array}{l}0.188 \\
0.275 \\
0.372 \\
\end{array}$ & $\begin{array}{l}0.190 \\
0.280 \\
0.378 \\
\end{array}$ & $\begin{array}{l}0.136 \\
0.206 \\
0.274 \\
\end{array}$ & & $\begin{array}{l}0.200 \\
0.296 \\
0.402 \\
\end{array}$ & $\begin{array}{l}0.200 \\
0.296 \\
0.402 \\
\end{array}$ & $\begin{array}{l}0.144 \\
0.212 \\
0.288 \\
\end{array}$ \\
\hline & $\begin{array}{l}2.1 \\
2.2 \\
2.3 \\
2.4 \\
\end{array}$ & $\begin{array}{l}0.416 \\
- \\
0.406 \\
0.404 \\
\end{array}$ & $\begin{array}{l}0.439 \\
0.456 \\
0.415 \\
0.411 \\
\end{array}$ & $\begin{array}{l}0.302 \\
0.940 \\
0.295 \\
0.298 \\
\end{array}$ & & $\begin{array}{l}0.422 \\
- \\
0.402 \\
0.396 \\
\end{array}$ & $\begin{array}{l}0.422 \\
0.448 \\
0.402 \\
0.396 \\
\end{array}$ & $\begin{array}{l}0.302 \\
1.21 \\
0.288 \\
0.284 \\
\end{array}$ \\
\hline & $\begin{array}{l}3.1 \\
3.3 \\
3.5 \\
\end{array}$ & - & $\begin{array}{l}0.86 \\
0.78 \\
0.64 \\
\end{array}$ & $\begin{array}{l}0.64 \\
0.75 \\
0.76 \\
\end{array}$ & & - & - & $\begin{array}{l}- \\
-\end{array}$ \\
\hline & $\begin{array}{l}4.1 \\
4.3 \\
4.5 \\
\end{array}$ & $\begin{array}{l}- \\
-\end{array}$ & $\begin{array}{l}0.89 \\
0.74 \\
0.66 \\
\end{array}$ & $\begin{array}{l}0.63 \\
0.67 \\
0.68 \\
\end{array}$ & & $\begin{array}{l}- \\
-\end{array}$ & $\begin{array}{l}0.512 \\
0.485 \\
0.442 \\
\end{array}$ & $\begin{array}{l}0.472 \\
0.543 \\
0.576 \\
\end{array}$ \\
\hline
\end{tabular}

Tabelle 14: Gemessene Dehnungsdifferenzen für Relativdurchbiegungen nach unten bis zugehörige Relativdurchbiegung nach oben (gemäss Tabellen 12 und 13) 


\begin{tabular}{|c|c|c|c|c|c|c|}
\hline \multirow[t]{2}{*}{ Teilphase } & \multicolumn{2}{|c|}{$\begin{array}{l}\text { Vorspanngrad des } \\
\text { betrachteten Schwing- } \\
\text { zyklus }\end{array}$} & \multicolumn{2}{|c|}{ dynamische Biegesteifigkeit } & \multicolumn{2}{|c|}{$\begin{array}{l}\text { Eigenfrequenz (bezogen auf Masse } \\
\text { in der Teilphase } 3.1 \text { bzw. } 4.1 \text { ) }\end{array}$} \\
\hline & $\kappa_{0}$ & $K_{\text {rel }}^{+}$ & $\begin{array}{l}\mathrm{B}_{\mathrm{dyn}} \cdot 10^{12} \\
{[\mathrm{~N} / \mathrm{mm} 2]}\end{array}$ & $\begin{array}{l}\text { Reduktion } \\
\text { zu } B_{\text {dyn, korr }}\end{array}$ & $\begin{array}{c}f \\
{[\mathrm{~Hz}]}\end{array}$ & Reduktion zu $f_{\text {korr }}$ \\
\hline $\begin{array}{l}3.1 \\
3.3 \\
3.5\end{array}$ & $\begin{array}{l}1.45 \\
0.91 \\
0.67\end{array}$ & $\begin{array}{l}0.69 \\
0.56 \\
0.48\end{array}$ & $\begin{array}{l}7.4 \\
6.35 \\
5.15\end{array}$ & $\begin{array}{l}11 \% \\
23 \% \\
38 \%\end{array}$ & $\begin{array}{l}4.25 \\
3.94 \\
3.54\end{array}$ & $\begin{array}{r}6 \% \\
12 \% \\
21 \%\end{array}$ \\
\hline $\begin{array}{l}4.1 \\
4.3 \\
4.5\end{array}$ & $\begin{array}{l}1.45 \\
0.91 \\
0.67\end{array}$ & $\begin{array}{l}0.69 \\
0.56 \\
0.48\end{array}$ & $\begin{array}{l}6.4 \\
6.1 \\
5.15\end{array}$ & $\begin{array}{l}11 \% \\
15 \% \\
28 \%\end{array}$ & $\begin{array}{l}3.95 \\
3.86 \\
3.54\end{array}$ & $\begin{array}{r}6 \% \\
8 \% \\
16 \%\end{array}$ \\
\hline $\begin{array}{l}3.1 \\
3.3 \\
3.5\end{array}$ & $\begin{array}{l}1.17 \\
0.80 \\
0.61\end{array}$ & $\begin{array}{l}0.64 \\
0.54 \\
0.47\end{array}$ & $\begin{aligned} 10.0 \\
7.25 \\
5.55\end{aligned}$ & $\begin{array}{l}19 \% \\
41 \% \\
55 \%\end{array}$ & $\begin{array}{l}4.45 \\
3.81 \\
3.33\end{array}$ & $\begin{array}{l}11 \% \\
23 \% \\
33 \%\end{array}$ \\
\hline $\begin{array}{l}4.1 \\
4.3 \\
4.5\end{array}$ & $\begin{array}{l}1.17 \\
0.80 \\
0.61\end{array}$ & $\begin{array}{l}0.64 \\
0.56 \\
0.47\end{array}$ & $\begin{array}{l}8.4 \\
6.9 \\
5.55\end{array}$ & $\begin{array}{l}16 \% \\
31 \% \\
45 \%\end{array}$ & $\begin{array}{l}4.05 \\
3.71 \\
3.23\end{array}$ & $\begin{array}{r}9 \% \\
17 \% \\
26 \%\end{array}$ \\
\hline
\end{tabular}

Tabelle 15: Reduktion der dynamischen Biegesteifigkeit und der Eigenfrequenz bei teilweiser Vorspannung bezịglich der korrigierten dynamischen Biegesteifigkeit $B_{\text {dyn, korr }}$ bzw. der korrigierten Eigenfrequenz $f_{\text {korr }}$ bei voller Vorspannung.

(Eigenfrequenz auf Einheitsmasse in der Teilphase $3.1 \mathrm{bzw} .4 .1$ umgerechnet, vgl. Bild 30) 